\title{
Characterisation of Denatured States and Reversible Unfolding of Sensory Rhodopsin II
}

Yi Lei $\operatorname{Tan}^{1}$, James Mitchell ${ }^{2}$, Judith Klein-Seetharaman², Daniel Nietlispach ${ }^{1 *}$

${ }^{1}$ Department of Biochemistry, 80 Tennis Court Road, University of Cambridge, CB2 1GA, United Kingdom

${ }^{2}$ Biomedical Sciences Division, Warwick Medical School, University of Warwick, Coventry, CV4 7AL, United Kingdom

*To whom correspondence should be addressed: Daniel Nietlispach, Department of Biochemistry, 80 Tennis Court Road, University of Cambridge, CB2 1GA, United Kingdom. Phone: +44 1223 766023, Fax: +44 1223 766002, Email: dn206@cam.ac.uk 


\section{Abstract}

Our understanding on the folding of membrane proteins lags behind that of soluble proteins due to challenges posed by the exposure of hydrophobic regions during in vitro chemical denaturation and refolding experiments. While different folding models are accepted for soluble proteins, only the two-stage model and the long-range interactions model have been proposed so far for helical membrane proteins. To address our knowledge gap on how different membrane proteins traverse their folding pathways, we have systematically investigated the structural features of SDS-denatured states and the kinetics for reversible unfolding of sensory rhodopsin II (pSRII), a retinal-binding photophobic receptor from Natronomonas pharaonis. pSRII is difficult to denature, and only SDS can dislodge the retinal chromophore without rapid aggregation. Even in 30\% SDS (0.998 $\left.X_{\text {SDS }}\right)$ pSRII retains the equivalent of six out of seven transmembrane helices, while the retinal binding pocket is disrupted, with transmembrane residues becoming more solvent-exposed. Folding of pSRII from an SDS-denatured state harbouring a covalently-bound retinal chromophore shows deviations from an apparent two-state behaviour. SDS-denaturation to form the sensory opsin apo-protein is reversible. We report pSRII as a new model protein which is suitable for membrane protein folding studies, and has a unique folding mechanism that differs from those of bacteriorhodopsin and bovine rhodopsin.

\section{Highlights}

- SDS-denatured pSRII remains helical but tertiary packing is disrupted.

- Refolding of retinal-bound SDS-denatured pSRII shows non-two-state kinetics.

- $\quad$ SSRII can be reversibly denatured in SDS to form sensory opsin apo-protein.

- pSRII has a different folding mechanism to bacteriorhodopsin and bovine rhodopsin.

- $\quad$ PSRII is established as a new model protein for membrane protein folding studies.

\section{Keywords}

Membrane proteins, Folding, Retinal, Kinetics

\section{Abbreviations}

CD, Circular Dichroism

c7-DHPC, Diheptanoylphosphatidylcholine

FRET, Förster resonance energy transfer

MRE, Mean residue ellipticity

pSRII, N. pharaonis Sensory rhodopsin II 
PSB, Protonated Schiff base

SDS, Sodium dodecyl sulphate

UV/vis, UV/visible 


\section{Introduction}

Membrane proteins constitute around $23 \%$ of the human proteome [1] and are involved in important functions, such as cell adhesion, signalling, molecule transport and catalysis. Misfolding, or failure of a protein to fold into its functional conformation, can lead to disruption of regulated cell functions and diseases through various mechanisms, including loss of function of the misfolded protein, cellular stress responses and cell death triggered by toxic accumulation of misfolded protein [2,3]. Membrane protein misfolding is implicated in the progression of various diseases such as cystic fibrosis, Charcot-Marie-Tooth disease and retinitis pigmentosa [4]. Insights on the folding mechanisms of membrane proteins might pave the way towards discovering new therapeutic approaches for membrane misfolding diseases. Protein denaturation in vitro offers an avenue for accessing selected conformations within the ensemble of structural states adopted by a protein whilst it is folding [5]. Hence, understanding the residual structure in the chemically denatured states of membrane proteins might provide mechanistic clues to how these proteins fold and misfold.

Advances in studying membrane protein folding progress slower than that for globular protein folding due to various experimental challenges [6]. In vitro studies require membrane proteins to be functionally reconstituted in a detergent or lipid environment, which often also renders them resistant toward denaturation. Another challenge is the formation of aggregates due to exposure of large hydrophobic regions upon membrane protein denaturation. Experimental conditions that were successfully applied to unfold a particular protein might not be applicable to others; hence, no general approach seems to be suited. Beta-barrel membrane proteins tend to be denatured using chaotropes. Alpha-helical membrane proteins are often resistant to chaotropes, while ionic detergents such as SDS have been shown to be efficient denaturants for some cases, though the proteins are likely to be recalcitrant to complete denaturation [7]. Characterisation of the denatured states, and the unfolding and folding pathways require maximal and reversible unfolding of the protein with minimal aggregation. Although reversible chemical denaturation has only been demonstrated for a handful of alpha-helical membrane proteins [8-17], biophysical, thermodynamic and kinetic studies have enabled extensive characterisation of the molecular nature of denatured states $[8,9,11,13,16-19]$, protein stability $[10,12,13,17,20]$, transition state [21,22], and folding intermediates [23,24], hence yielding valuable insights on the folding mechanisms of alpha-helical membrane proteins. 
A widely held concept for explaining the folding of integral alpha-helical membrane proteins is the two-stage model originally proposed by Popot and Engelman [25]. This model postulates that transmembrane helices are first formed independently after insertion into the membrane environment, followed by formation of tertiary packing during the second stage. The large retention of secondary structure in the SDS-denatured state of bacteriorhodopsin appears to support the idea that individual secondary structure elements can be stabilised in a micellar environment and are formed before native tertiary packing is established. However, the notion of individual transmembrane helices folding independently in a membrane environment has been partly questioned by structural studies on isolated helical fragments of bacteriorhodopsin, which show that C-terminal helices are only partially helical and require the presence of $\mathrm{N}$-terminal helices to fold [26], hence implying that tertiary contacts are necessary for some transmembrane helices to acquire stable folds. The folding of rhodopsin deviates even further from the two-stage model. SDS-denatured rhodopsin reveals extensive abolishment of helicity and native tertiary interactions, and the formation of largely unfolded structures with some residual structure elements causing burial of specific cysteines and tryptophans [27]. Cell-based [28] and in vitro [27,29,30] folding studies on rhodopsin suggest that interactions between the extracellular and transmembrane domains constitute a 'folding core' which is important in the early stages of folding. Single-molecule dynamic force spectroscopy reveals a core of rigid structural elements in bovine rhodopsin but not in bacteriorhodopsin [29]. NMR and EPR studies of SDS-denatured rhodopsin show flexible cytoplasmic and extracellular ends while some residual structure remains in the transmembrane and extracellular regions [30]. The distributions of rigid and flexible segments in rhodopsin identified in these experiments are consistent with the results from simulated thermal unfolding studies using the Floppy Inclusions and Rigid Substructure Topography (FIRST) method. In comparison, simulation with bacteriorhodopsin shows clusters, which align with individual helices, breaking off early in the simulation. The unfolding mechanism of bacteriorhodopsin therefore illustrates better consistency with the two-stage model [31]. The more recent "long-range interactions model" [32] has been proposed in order to emphasise the importance of interactions between residues which are far apart in sequence but come close in space during early stages of protein folding.

Sensory rhodopsin II (pSRII), from the archaeon Natronomonas pharaonis, functions as a repellent phototactic receptor to blue light via isomerisation of its all-trans retinal chromophore to the 13-cis conformation, enabling the archaeon to seek the dark when respiratory substrates are plentiful. Simulated thermal unfolding experiments suggest that pSRII is stabilised by a combination of mechanisms found for rhodopsin and bacteriorhodopsin [31], with a subset of helices breaking off early in the simulation and a 
core involving residues from other helices and loops remaining until late. More experimental studies are needed to understand the folding pathway of membrane proteins, particularly how and when secondary and tertiary structure elements are being formed. Retinal-binding seven transmembrane helical proteins are excellent models for these studies, since the retinal chromophore acts as a natural reporter of the binding pocket and thus, the tertiary structure.

In this paper, we have characterised the SDS-denatured states and kinetics for the unfolding and refolding of pSRII in SDS/diheptanoylphosphatidylcholine (c7-DHPC) mixed detergent micelles. Extensive screening of different denaturants and additives identified SDS as the most suitable denaturant for studying the unfolding of pSRII. Circular dichroism, UV-visible spectroscopy and tryptophan fluorescence show that the SDS-denatured state is characterised by loss of tertiary structure (inter-helical contacts) and little loss in secondary structure, similar to bacteriorhodopsin and hence distinguishing pSRII from the long-range interactions model. The native chromophore can be reformed from a SDS-denatured state with retinal remaining covalently attached and from the apo-protein state, showing that both protein folding and retinal attachment are reversible. Notable differences were found in the kinetics of SDS denaturation and refolding of pSRII compared to bacteriorhodopsin and other membrane proteins which fold via a two-state mechanism (i.e. no intermediates between folded and unfolded states), suggesting marked differences in the unfolding and folding pathways. Overall, this study has established pSRII as a new model protein for membrane protein folding studies.

\section{Results}

\section{Denaturant and additives screening identify SDS as the most suitable denaturant for unfolding pSRII}

To identify the most suitable denaturant for unfolding pSRII, the extent of protein unfolding was determined by monitoring changes in the UV/vis absorbance of the retinal chromophore. The results are summarised in Fig. 1 and Table S1. The exquisite sensitivity of the retinal UV/vis absorbance fine structure enables facile monitoring of structural changes in the retinal binding pocket during pSRII denaturation and detection of unfolding intermediates. In pSRII, all-trans retinal is covalently linked to Lys-205 via a protonated Schiff base (PSB) (Fig. 1a), and gives rise to a main absorbance peak at $498 \mathrm{~nm}$, with vibronic side bands at 
$460 \mathrm{~nm}, 420 \mathrm{~nm}$ and $370 \mathrm{~nm}$ (Fig. 1b) [33]. Retinal PSB devoid of native protein interactions absorbs at $440 \mathrm{~nm}\left(\mathrm{SR}_{440}\right)$ [34]. Schiff base hydrolysis leads to the release of free all-trans retinal, which absorbs at $390 \mathrm{~nm}\left(\mathrm{SO}_{390}\right)$ [35], from the sensory opsin apo-protein.

pSRII was subjected to denaturation for 1 day at room temperature in the presence of different denaturants (Fig. 1C-e). Only sodium dodecyl sulphate (SDS), an anionic detergent, yielded complete unfolding and Schiff base hydrolysis, with only $\mathrm{SO}_{390}$ being observed (Fig. 1d). Like bacteriorhodopsin [36], pSRII cannot be denatured by urea and guanidine hydrochloride, whereas bovine rhodopsin is susceptible to both denaturants [37]. Trifluoroacetic acid led to the formation of $\mathrm{SR}_{440}$ which slowly decayed to form $\mathrm{SO}_{390}$ over the course of two weeks (results not shown), indicating that the rate of Schiff base hydrolysis is reduced considerably in an acidic environment.

Aggregation was assessed by SDS-PAGE without boiling of the samples (for details see Methods and Supplementary Information). Fig. 2 shows a silver-stained SDS-PAGE gel for pSRII in $0 \%, 0.5 \%$ and $3 \%$ SDS in the presence of $0.1 \%$ c7-DHPC for $0-72$ hours. Native pSRII in 0.1\% c7-DHPC ran as a major monomeric band and a minor dimeric band. Sedimentation velocity analytical ultracentrifugation of $4.9 \mu \mathrm{M}$ pSRII in $0.1 \%$ c7-DHPC confirmed that the monomeric species accounts for more than $90 \%$ of the total loading sample while the dimeric species accounts for about $4 \%$ [38], consistent with the relative intensities of the two bands on SDS-PAGE gel. The amount of oligomerisation increased over prolonged unfolding time (Fig. 2), and correlated with the loss of alpha-helical structure and increase in beta-sheet structure over time (Fig. S1b). pSRII showed SDS concentration-dependent oligomerisation (Fig. 2). While dimers and higher-order oligomeric species were observed in both $0.5 \%$ and $3 \%$ SDS, the amount of higher oligomeric states was lower in $3 \%$ SDS. This suggests that $0.5 \%$ SDS induced slow or partial unfolding, and the exposed protein surfaces led to preferential formation of higher oligomers. pSRII remained predominantly monomeric and the amount of aggregation did not show noticeable increase after 2 hours in SDS, which is the typical timeframe for biophysics and kinetics studies reported in this paper. The amounts of dimers and higher-order oligomers steadily increased from 24 hours onwards in both $0.5 \%$ and $3 \%$ SDS.

Further attempts at optimising unfolding conditions (see Supplementary Information and Table S2) in the presence of different additives and solvent conditions (salt concentration, $\mathrm{pH}$ ) identified SDS at $\mathrm{pH} 6.0$ and SDS at $\mathrm{pH} 2.0$ as conditions which yielded maximal unfolding and the least amount of aggregation. Further biophysical studies were therefore pursued in different SDS concentrations at $\mathrm{pH} 6.0$ and $\mathrm{pH} 2.0$ to study structural changes 
and kinetics of pSRII unfolding. Described below are the results obtained at $\mathrm{pH}$ 6.0. The results obtained at $\mathrm{pH} 2.0$ are described in Supplementary Information. The results indicate that protein structural changes and unfolding kinetics at both $\mathrm{pHs}$ are dependent on molar fraction of SDS ( $X_{\mathrm{SDS}}$ ), while Schiff base hydrolysis was found to be much slower at $\mathrm{pH} 2.0$ than at $\mathrm{pH} 6.0$.

Based on the combined criteria of the largest degree of denaturation and the least amount of aggregation, in subsequent sections, structural properties of SDS-denatured pSRII were studied at a maximum of 2 hours after initial exposure to SDS, thus ensuring complete unfolding and negligible amounts of aggregates accumulated across all conditions. Since the extent of membrane protein unfolding correlates with $X_{\text {SDS }}$ [20], high $X_{\text {SDS }}$ in low c7-DHPC concentration $(0.1 \% \mathrm{c} 7-\mathrm{DHPC})$ was used in order to obtain maximally unfolded pSRII for biophysical characterisation of SDS-denatured states. The kinetics of unfolding and refolding reactions were studied in the presence of excess concentrations of SDS/c7-DHPC mixed micelles (SDS $+0.5 \% \mathrm{c} 7$-DHPC) to obtain accurate rate constants while avoiding multiple micelle occupancy. Control experiments show that similar secondary and tertiary structure changes were yielded for SDS-denatured pSRII in the presence of $0.1 \% \mathrm{c} 7$-DHPC vs. $0.5 \%$ c7-DHPC (Fig. S1e-f).

\section{Secondary structure changes}

The loss of secondary structure in pSRII, unfolded for 2 hours at $\mathrm{pH} 6.0$ with $0-0.998 X_{\text {SDS }}$ (0-30\% SDS in $0.1 \%$ c7-DHPC), was monitored by circular dichroism spectroscopy. Little change was observed, suggesting that alpha-helical content was largely preserved in SDS-denatured pSRII at pH 6.0 (Fig. 3a) and at pH 2.0 (Fig. S4). Similar changes in alpha-helical content were observed for pSRII unfolded in SDS + 0.5\% c7-DHPC (Fig. S1e). Changes in mean residue ellipticity (MRE) at $222 \mathrm{~nm}$ from folded pSRII can be divided into two distinct stages: $X_{\text {SDS }}$ up to 0.980 (3\% SDS in $0.1 \%$ c7-DHPC) led to a decrease in MRE magnitude by up to $12.6 \pm 1.0 \%$; further increase in $X_{\text {SDS }}$ reversed this trend, with only $9.3 \pm$ $1.6 \%$ decrease in MRE magnitude observed at $0.998 X_{\text {SDS }}(30 \%$ SDS in $0.1 \%$ c7-DHPC) (Fig. 3b). This slight increase in helicity at high $X_{\text {SDS }}$ could be attributed to SDS favouring the formation of non-native helical structures [39].

Changes in different secondary structure elements calculated using CDPro software [40] are shown in Table S3 and Fig. S5. The fraction of regular helix $(H(r))$ experienced the greatest changes, showing significant decrease in $>0.834 X_{\mathrm{SDS}}$ at $\mathrm{pH} 6.0$ and $\mathrm{pH} 2.0$. The fraction of 
distorted helix $(H(d))$ increased in $>0.893 X_{S D S}$ at $\mathrm{pH} \mathrm{6.0,} \mathrm{but} \mathrm{did} \mathrm{not} \mathrm{show} \mathrm{significant}$ changes at $\mathrm{pH}$ 2.0. The content of other secondary structure elements (regular sheet, distorted sheet, turn and unordered) showed only slight increase in $>0.834 X_{\text {SDS }}$ at $\mathrm{pH} 6.0$ and $\mathrm{pH} 2.0$.

\section{Changes in the Retinal Binding Pocket}

UV/vis spectra of the retinal chromophore were recorded to monitor structural changes at the retinal binding pocket (Fig. $4 a)$. In low $X_{\mathrm{SDS}}\left(\leq 0.893 X_{\mathrm{SDS}} ; 0.5 \%\right.$ SDS in $\left.0.1 \% \mathrm{c} 7-\mathrm{DHPC}\right)$, an isosbestic point at $\sim 420 \mathrm{~nm}$ was observed. A spectral intermediate absorbing at $440 \mathrm{~nm}$ $\left(\mathrm{SR}_{440}\right)$, characteristic of a PSB devoid of native retinal-protein contacts, appeared in $\geq 0.943$ $X_{\text {SDS }}(1 \%$ SDS in $0.1 \%$ c7-DHPC).

To accurately determine the unfolding transition, equilibrium unfolding measurements were performed by unfolding pSRII in SDS in the presence of 0.5\% c7-DHPC and monitoring the loss of absorbance at $498 \mathrm{~nm}$ after 10 minutes (Fig. 4b). 0.5\% c7-DHPC was used to avoid multiple micelle occupancy by ensuring an excess molar ratio of detergent micelles to protein across all $X_{\mathrm{SDS}}$. The transition midpoint was around $0.81 X_{\mathrm{SDS}}$, higher than those reported for bacteriorhodopsin [20,41], indicating that pSRII in c7-DHPC is more stable than bacteriorhodopsin.

Each UV/vis spectrum was deconvoluted by non-linear least squares fitting of the chromophore absorbance peaks to a model of Gaussian peaks (Fig. S10c). Gaussian-fitted $\lambda_{\max }$ of dead-time spectra in different $X_{\mathrm{SDS}}$ (dead-time $=25 \mathrm{~s}$ ) revealed $X_{\mathrm{SDS}}$-dependent red shift of the chromophore absorbance peak which closely matches the increase in unfolded protein fraction obtained from equilibrium unfolding measurements (Fig. 4b). Since the $\lambda_{\max }$ values of protonated retinylidene model Schiff bases are sensitive to the proximity of the counteranion [42], spectral red-shift of the retinal chromophore could suggest an increase in the separation of the Schiff base counterion Asp-75 from the PSB upon denaturation of pSRII in SDS. The fitted $\lambda_{\max }$ remained constant beyond $0.943 X_{\text {SDS }}$ (5\% SDS in $0.5 \%$ c7-DHPC), suggesting that further increases in $X_{\text {SDS }}$ did not yield further separation of Asp-75 from the PSB, and the binding pocket is already fully opened.

\section{Tertiary Structure Changes}


pSRII contains 6 tryptophan residues, most of which are located in close proximity to the retinal chromophore (Fig. 5a). Their intrinsic fluorescence is quenched due to very efficient energy transfer to the retinal by FRET [43]. Fig. 5b shows the emission spectra of pSRII denatured in different $X_{\mathrm{SDS}}$ at $\mathrm{pH} 6.0$ for 2 hours. Increased tryptophan fluorescence intensities were observed, as Schiff base hydrolysis leads to removal of retinal from the binding pocket and away from the tryptophan residues. Tryptophan fluorescence of folded pSRII ( $0 X_{\text {SDS }}$ ) has $\lambda_{\max }$ of $337 \mathrm{~nm}$, which became gradually red-shifted with increasing $X_{\text {SDS }}$ up to $343 \mathrm{~nm}$ in $0.998 X_{\mathrm{SDS}}(30 \%$ SDS in $0.1 \% \mathrm{c} 7-\mathrm{DHPC})$. This indicates that some tryptophan residues have become more solvent-exposed upon protein unfolding. pSRII unfolded in SDS + 0.5\% c7-DHPC (i.e. the conditions chosen for the kinetic studies) yielded similar observations (Fig. S1f).

\section{Solvent Accessibility of the Binding Pocket}

Hydroxylamine serves as a probe for monitoring protein conformational changes near the Schiff base in retinal-binding proteins [44,45]. Hydroxylamine becomes the preferred nucleophile for Schiff base cleavage instead of water, leading to the cleavage of the PSB and formation of retinal oxime, which absorbs at $360 \mathrm{~nm}$ (Fig. 6a) [46]. In the presence of 10 $\mathrm{mM}$ hydroxylamine, pSRII in $0 X_{\mathrm{SDS}}$ at $\mathrm{pH} 6.0$ exhibited slow retinal PSB hydrolysis, suggesting that the retinal binding pocket is relatively solvent-inaccessible (Fig. $6 b(i)$ ). The rate of chromophore bleaching increased significantly in the presence of $0.980 X_{\text {SDS }}(3 \%$ SDS), with complete formation of retinal oxime within 30 minutes (Fig. 6b(ii)). This indicates that SDS leads to an increase in solvent-accessibility of the retinal binding pocket. Tryptophan fluorescence spectra of hydroxylamine-bleached pSRII, both in the absence and presence of SDS, showed increase in fluorescence intensity and $X_{\text {SDS-dependent red-shift of }}$ the peak (Fig. 6c), consistent with the release of retinal oxime and increase in solvent-accessibility of the binding pocket. In contrary to bacteriorhodopsin and bovine rhodopsin where the bleaching of purple membranes and bovine rod outer segment disk membrane in the presence of hydroxylamine did not lead to appreciable changes in the far-UV circular dichroism spectra $[47,48]$, the presence of hydroxylamine led to greater loss of pSRII secondary structure beyond the unfolding transition while the transition point remained the same (Fig. 6d). This suggests that unlike in bacteriorhodopsin and bovine rhodopsin, some secondary structure elements of pSRII might be stabilised by the presence of retinal covalent attachment and native retinal-protein interactions. 


\section{Kinetic measurements of pSRII unfolding}

The results of our biophysical studies show that pSRII unfolds in SDS to form $\mathrm{SO}_{390}$ via a $\mathrm{SR}_{440}$ intermediate, as summarised in Scheme 1:

$$
\mathrm{pSRIII} \underset{k_{1}}{\stackrel{k_{u}}{\rightleftharpoons}} \mathrm{SR}_{4<0} \stackrel{k_{n}}{\rightarrow} \mathrm{SO}_{392}
$$

where $k_{\mathrm{u}}, k_{\mathrm{f}}$ and $k_{\mathrm{h}}$ are the first-order rate constants for unfolding, folding and retinal Schiff base hydrolysis, respectively.

In the $\mathrm{SO}_{390}$ state, most of the secondary structure remains while tertiary structure is disrupted.

Since high $X_{\mathrm{SDS}}$ were used for the unfolding reactions $\left(k_{\mathrm{u}}>>k_{\mathrm{f}}\right)$, the unfolding model could be further simplified to yield Scheme 2:

$$
\mathrm{pSRII} \stackrel{k}{\rightarrow} \mathrm{SR}_{4<0} \stackrel{k_{11}}{\rightarrow} \mathrm{SO}_{393}
$$

To gain further insights into the unfolding pathway, the kinetics of pSRII unfolding were monitored by UV/vis spectroscopy and the time courses fitted using non-linear least squares methods (see Methods). Kinetic studies were done in the presence of excess concentrations of SDS/c7-DHPC mixed micelles (SDS $+0.5 \%$ c7-DHPC) to obtain accurate rate constants and to avoid multiple micelle occupancy.

Two rate constants, $k_{\mathrm{u}}$ and $k_{\mathrm{h}}$, were extracted, describing changes in retinal chromophore absorbance when pSRII was unfolded in SDS at pH 6.0 (no hydroxylamine), as depicted in Scheme 2. $k_{\mathrm{u}}$, the first-order rate constant for the formation of $\mathrm{SR}_{440}$, increases with increasing $X_{\text {SDS }}($ Table 1$) . k_{\mathrm{u}}$ is $(44 \pm 9) \times 10^{-4} \mathrm{~s}^{-1}$ in $0.893 X_{\mathrm{SDS}}(2.5 \%$ SDS in $0.5 \%$ c7-DHPC), about 120-fold smaller than the bacteriorhodopsin unfolding rate constant of 0.54 $\mathrm{s}^{-1}$ in $0.882 X_{\mathrm{SDS}}$ in SDS/DMPC/CHAPSO micelles reported by Curnow and Booth [20]. Bovine rhodopsin unfolds in $0.972 X_{\text {SDS }}(1 \%$ SDS in $0.05 \%$ DDM) with the time constant 0.32 $\pm 0.01 \mathrm{~s}$ [27], corresponding to $k_{\mathrm{u}}$ of $3.125 \mathrm{~s}^{-1}$, which is about 700 -fold larger than for the unfolding of pSRII. Plotting the natural logarithm of $k_{\mathrm{u}}$ against $X_{\mathrm{SDS}}$ yields the unfolding arm 
of the chevron plot (Fig. 7c), where $\ln k_{\mathrm{u}}$ increases linearly against $X_{\mathrm{SDS}}$. Minor non-linearity observed in high $X_{\mathrm{SDS}}$ can be attributed to the formation of cylindrical micelles at high SDS concentrations $[49,50]$.

$k_{\mathrm{h}}$, the rate constant of Schiff base hydrolysis, is independent of SDS concentration (Table 1). $k_{\mathrm{h}}$ is $(4.2 \pm 0.6) \times 10^{-4} \mathrm{~s}^{-1}$ in $0.834-0.971 X_{\mathrm{SDS}}(1.5-10 \%$ SDS in $0.5 \% \mathrm{c} 7-\mathrm{DHPC})$, similar to but slightly smaller than $\sim 9 \times 10^{-4} \mathrm{~s}^{-1}$, the rate constant of free retinal formation from the 440-nm state of bacteriorhodopsin $\left(b_{440}\right)$ reported by Curnow and Booth [20]. This suggests that $\mathrm{SR}_{440}$ and $\mathrm{bR}_{440}$ could have similar solvent accessibility to their binding pockets, but $k_{\mathrm{h}}$ might also be rate-limited by other factors such as the reactivity of the Schiff base, possibly due to different unfolding conditions being used in these studies.

\section{Refolding from $\mathrm{SR}_{440}$}

Reversibility of in vitro unfolding is important for determining whether the denatured states are on-pathway species. Successful refolding was assessed by recovery of the native chromophore absorbance at $498 \mathrm{~nm}$ with its characteristic vibronic side bands at $460 \mathrm{~nm}$, $420 \mathrm{~nm}$ and $370 \mathrm{~nm}$. Unfolded pSRII with retinal remaining covalently attached $\left(\mathrm{SR}_{440}\right)$ was first attained by incubating pSRII in $0.943 X_{\text {SDS }}(5 \%$ SDS $+0.5 \%$ c7-DHPC) for 4 minutes. Refolding was then initiated by 12 -fold dilution of $\mathrm{SR}_{440}$ into refolding buffer containing different $X_{\mathrm{SDS}}$ and monitored by UV/vis spectroscopy. All refolding kinetics were measured in the presence of $0.5 \% \mathrm{c} 7$-DHPC to minimise effects due to multiple micelle occupancy. Refolding buffer included $0.500-0.700 X_{\text {SDS }}(0.30-0.70 \%$ SDS) for measuring refolding rates in $0.698-0.779 X_{\text {SDS }}(0.69-1.06 \%$ SDS $)$.

Absorption spectra were taken at fixed time points during refolding. Fig. $7 a$ shows the spectra recorded in $0.698 X_{\mathrm{SDS}}$, where the rate constant of folding $\left(k_{\mathrm{f}}\right)$ was significantly greater than the rate constant of retinal hydrolysis $\left(k_{\mathrm{h}}\right)$, and in $0.779 X_{\mathrm{SDS}}$, where $k_{\mathrm{f}}$ was similar to $k_{\mathrm{h}}$. In both cases, as the reaction progressed, absorbance at $440 \mathrm{~nm}$ decreased while absorbance at $390 \mathrm{~nm}$ and $\sim 500 \mathrm{~nm}$ increased simultaneously, albeit at different rates. This indicates that formation of folded pSRII is fast whereas formation of $\mathrm{SO}_{390}$, either directly from $\mathrm{SR}_{440}$ or from slow unfolding of refolded pSRII, is slow and observable within 2 hours only in $\geq 0.745 X_{\text {SDS. }}$. For $\leq 0.723 X_{\text {SDS }}$, the time dependence of pSRII recovery was approximated with a single exponential model $\left(k_{\mathrm{f}}>>k_{\mathrm{h}}\right)$ (Fig. S11a-c). For $\geq 0.745 X_{\mathrm{SDS}}$, a double exponential model with the experimental rate constants $R_{1}$ and $R_{2}$ would be required 
(Figs. $7 \mathrm{~b} \& \mathrm{~S} 11 \mathrm{~d}-\mathrm{f}$ ), and $k_{\mathrm{f}}$ and $k_{\mathrm{u}}$ were calculated from the experimentally determined $\mathrm{R}_{1}$, $\mathrm{R}_{2}$ and $k_{\mathrm{h}}$ using Equations 12 and 13 (see Methods). Based on experimental unfolding kinetic measurements, $k_{\mathrm{h}}$ is independent of $X_{\mathrm{SDS}}$ (Table 1$)$, and is taken as $(3.72 \pm 0.17) \times$ $10^{-4} \mathrm{~s}^{-1}$ for $0.834 X_{\mathrm{SDS}}$ in $0.5 \% \mathrm{c} 7$-DHPC. It is noted that values of $k_{\mathrm{u}}$ obtained from refolding kinetic measurements tend to be unreliable, as they are dominated by errors in $k_{\mathrm{f}}$ and $k_{\mathrm{h}}$.

In contrast to the unfolding arm of the chevron plot $\ln \left(k_{\mathrm{f}}\right.$ and $\ln k_{\mathrm{u}}$ against $\left.X_{\mathrm{SDS}}\right)$ which is essentially linear, the folding arm showed clear deviation from a linear trend ('roll-over') (Fig. 7c). This result strongly indicates that formation of tertiary structure packing during refolding of $\mathrm{SR}_{440}$ is more complex than the two-state kinetics that has been observed for the equivalent process in bacteriorhodopsin [20,51]. There is no evidence for aggregation or multiple micelle occupancy being the origin of non-linearity in the folding arm (see Fig. S13 and Supplementary Results), hence further supporting a more complex folding mechanism for pSRII.

\section{Refolding from $\mathrm{SO}_{390}$ state}

Refolding from the $\mathrm{SO}_{390}$ apo-protein state was explored to determine whether retinal Schiff base hydrolysis is reversible. $\mathrm{SO}_{390}$ was first attained by incubating pSRII in $0.893 \mathrm{X}_{\mathrm{SDS}}$ ( $2.5 \%$ SDS in $0.5 \%$ c7-DHPC) for 2 hours at room temperature. Retinal was not removed after unfolding and no additional retinal was introduced. Refolding was initiated by 12-fold dilution of $\mathrm{SO}_{390}$ into refolding buffer and monitored for 2 hours by UV/vis spectroscopy. The refolding buffer included $0-0.294 X_{\text {SDS }}(0-0.125 \%$ SDS in $0.5 \%$ c7-DHPC) to enable measurement of refolding kinetics in $0.410-0.519 X_{\text {SDS }}(0.208-0.323 \%$ SDS in $0.5 \%$ c7-DHPC).

Time-courses of refolding from $\mathrm{SO}_{390}$ showed an isosbestic point at $420 \mathrm{~nm}$ (Fig. 8a), suggesting that the rate-limiting step of the reaction is the reformation of retinal Schiff base, assuming that the reaction proceeds through $\mathrm{SR}_{440}$ (i.e. same pathway as unfolding).

Time-courses of refolding from $\mathrm{SO}_{390}$ were modelled to Scheme 3 as pseudo first-order reactions:

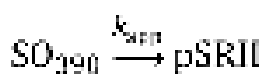


where $k_{\text {app }}$ is the apparent rate constant for the reconstitution of the native chromophore, taking into account both retinal re-attachment (reformation of the Schiff base) and protein folding.

$k_{\text {app }}$ in $0.410-0.519 X_{\text {SDS }}$ ranged from $1.7 \times 10^{-4} \mathrm{~s}^{-1}$ to $12.9 \times 10^{-4} \mathrm{~s}^{-1}$ (Table 3), similar to the rate constant for refolding of bacteriorhodopsin from $\mathrm{bO}_{390}$ of $17 \times 10^{-4} \mathrm{~s}^{-1}$ in $0.472 X_{\mathrm{SDS}}$ reported by Curnow and Booth [20]. This suggests that the rate of refolding from $\mathrm{SO}_{390}$ is limited by Schiff base reformation. The natural logarithm of $k_{\text {app }}$ (In $k_{\text {app }}$ ) appeared to be linearly-dependent against $X_{\mathrm{SDS}}$ in $0.410-0.519 X_{\mathrm{SDS}}$ (Fig. 8b), though the fast $k_{\text {app }}$ in $<0.4$ $X_{\text {SDS }}$ has precluded its measurement by means of manual mixing.

Overnight incubation of the refolding reaction at room temperature led to a substantial increase in the amount of refolded pSRII compared to the recovery yield obtained after two hours (Fig. S14a). This additional slow phase for native chromophore recovery might suggest that there is a small population of misfolded $\mathrm{SO}_{390}$ which requires some structural rearrangements to be capable of rebinding retinal and hence refolds at a much slower timescale.

Refolding yields were estimated by calculating the concentration of free retinal at the projected plateau of the single exponential fitted for the decrease in $\mathrm{SO}_{390}$ over the two-hour refolding time-course (Fig. S12). Refolding yields decreased with increasing amount of time (2-72 hours) during which pSRII remained unfolded in $0.893 X_{\text {SDS }}$, and correlated with the monomeric population left in the unfolded reaction assessed by SDS-PAGE (Fig. S1d: Lanes 6-10) and quantified using Image (Fig. 8c). Hence, only monomeric $\mathrm{SO}_{390}$ was capable of refolding to yield the native chromophore while the oligomeric species were irrecoverable misfolded off-pathway species. The refolding rate constant was shown to be independent of the time spent in the $\mathrm{SO}_{390}$ state and the extent of aggregation (Fig. S14b).

\section{Discussion}

Membrane proteins are notoriously difficult to denature due to their inherent stability once functionally reconstituted into membrane mimetics and their tendency to aggregate if the hydrophobic mimetic environment were perturbed. The studies presented in this paper first employed the screening of different denaturants and additives to simultaneously maximise 
the extent of unfolding in pSRII and minimise aggregation of the unfolding intermediates and unfolded states. SDS was identified as the most suitable condition for pSRII unfolding studies, causing non-specific aggregation only after prolonged exposure of the protein to SDS over several days. Using various biophysical techniques (CD, UV/vis spectroscopy, tryptophan fluorescence), the molecular properties of the denatured states were characterised within 2 hours of SDS exposure, which is the minimum time required to fully denature pSRII across all $X_{\text {SDS }}$ with minimal aggregation. SDS-denatured pSRII preserved much of its helical secondary structure whilst its tertiary structure was largely disrupted, as evidenced by loss of the retinal chromophore and changes in tryptophan fluorescence intensities. The kinetics of unfolding and refolding reactions were characterised, yielding the chevron plot and showing pSRII to be a model protein with complex folding mechanism.

\section{Denaturant screening}

SDS was identified as the most suitable condition for unfolding pSRII. Non-specific aggregation was not observed within the typical timeframe of biophysical and kinetics studies (2 hours), despite being visible in the form of soluble oligomers over much longer timescale of days. While some guidelines are available for minimising aggregation mostly in the contexts of globular proteins, intrinsically disordered proteins, and protein refolding [5254], general recommendations for minimising aggregation of membrane proteins under denaturing conditions are lacking. The motivation behind screening different denaturants and additives is to find conditions which favour unfolding and disfavour aggregation. We explored typical methods for shielding exposed hydrophobic surfaces and balancing surface charges, but the case of SDS-denatured pSRII proved to be more complex. This is a challenging problem because the same driving forces, namely formation of hydrogen bonds and burial of hydrophobic surfaces, are involved in both aggregation and protein folding [52]. A compromise was found in which aggregation was minimised without limiting the extent of unfolding by conducting biophysical and kinetics investigations at shorter time periods ( 2 hours).

Previous unfolding studies of bacteriorhodopsin and bovine rhodopsin also identify SDS as the most suitable denaturant, and give rise to many biophysical and structural studies on the SDS-denatured states of these two proteins. The striking similarities in the properties of bacteriorhodopsin and pSRII in the presence of different denaturants (urea, guanidine hydrochloride, SDS mixed micelles at neutral and acidic $\mathrm{pHs}$ ) suggest that the two proteins might have comparable unfolding mechanisms [36]. In contrast, the effects of denaturants on 
bovine rhodopsin are very different: urea and guanidine hydrochloride both unfold rhodopsin but lead to aggregation, while SDS alone and $3 \%$ SDS $+8 \mathrm{M}$ urea unfold rhodopsin to different extents, both without causing aggregation [37]. These differences in the response to different denaturants and the presence of aggregates in pSRII but not rhodopsin upon denaturation in SDS suggest different folding/unfolding pathways of pSRII vs. rhodopsin.

\section{SDS-denatured states of pSRII}

Overall, structural changes in pSRII upon denaturation in SDS both at $\mathrm{pH} 2.0$ (Supplementary Results) and at pH 6.0 (main text) included an increase in accessibility of the retinal binding pocket with concomitant larger exposure of this region to solvent up to $0.943 X_{\text {SDs. }}$. Most of the transmembrane helical structure was preserved, and the small loss of alpha-helicity was attributed to fraying of helix ends. Beyond $0.994 X_{\mathrm{SDS}}$, non-native helical structure and burial of tryptophan residues were observed, likely because of the helix-inducing effect of the cylindrical SDS micelle environment wrapping around the protein $[23,50]$. The unfolding transition is comparable across the three biophysical techniques (Fig. 3b, Fig. 4b \& Fig. S9). Extensive comparison of unfolding rate constants extracted using different biophysical techniques was done for pSRII unfolding in SDS + 0.1\% c7-DHPC (Fig. S8). For detailed comparisons of pSRII unfolding in $\mathrm{pH} 2.0$ vs. $\mathrm{pH} 6.0$ and for the comparison of unfolding rate constants across different biophysical techniques, see Supplementary Discussion.

It is noticeable that pSRII unfolded much slower than both bacteriorhodopsin and bovine rhodopsin. This appears to contradict existing knowledge on the compactness of the native states of these proteins. Rhodopsin is the most compact and has limited susceptibility to bleaching even in the presence of $50 \mathrm{mM}$ hydroxylamine [55], while micellar suspensions of monomeric bacteriorhodopsin [45] and pSRII (Fig. 6b(i)) showed detectable bleaching. While the utilisation of different detergent mixed micelle systems complicates a direct comparison of studies on the three proteins (c7-DHPC for pSRII, DMPC/CHAPS for bacteriorhodopsin, and DDM for bovine rhodopsin), this factor is unlikely to solely account for the large differences in unfolding rate constants. Preliminary studies of SDS-mediated unfolding of pSRII reconstituted in DDM yielded comparable rate constants for protein unfolding and Schiff base hydrolysis as those for pSRII in SDS/c7-DHPC (Fig. S15). Altogether, comparative studies on the SDS-denatured states of pSRII, bacteriorhodopsin and bovine rhodopsin point towards differences in stability, unfolding mechanisms and protein-SDS interactions amongst the three proteins. More systematic comparisons using comparable 
conditions and membrane mimetics will be necessary to make more assertive conclusions on differences in kinetics and thermodynamic parameters between these proteins. Further investigation into the properties of mixed micelles and high-resolution studies on protein-detergent interactions in SDS-denatured pSRII will provide further valuable insights into the molecular nature of the denatured states and the unfolding/folding pathway.

\section{Folding Model for pSRII}

Although the SDS-denatured apo-protein states of both bacteriorhodopsin and pSRII retain significant amounts of secondary structure and no native retinal-protein interactions, there are fundamental differences in their unfolding/folding pathways and protein-SDS interactions. This was most notably exemplified by non-linearity of the folding arm in the pSRII chevron plot, suggesting either transient aggregation or the presence of 'kinetically-invisible' intermediate(s) in the folding of pSRII but not in bacteriorhodopsin. Given there is no dependence of the refolding rate constant on pSRII or detergent concentration (see Supplementary Results), transient aggregation and multiple micelle occupancy are unlikely, suggesting a complex folding pathway between $\mathrm{SR}_{440}$ and pSRII with on/off-pathway intermediate(s). It is difficult to reach a definitive conclusion about the existence and properties of the putative folding intermediate(s). Even for globular proteins, interpretations of non-linear chevron plots and conclusions about the presence and properties of intermediate states are often elusive and require extensive analyses of phi-values in different mutants. Nevertheless, it is evident that the folding pathway between $\mathrm{SR}_{440}$ and folded pSRII, which predominantly involves the formation of native tertiary packing, shows clear deviations from the two-state model exemplified by bacteriorhodopsin.

Retinal binding was shown to be reversible and the refolding yield from $\mathrm{SO}_{390}$ correlated with the amount of monomeric $\mathrm{SO}_{390}$ left after prolonged incubation in SDS over several days. Reversible unfolding of the apo-protein state can be achieved for bacteriorhodopsin and pSRII, both of which retain significant portions of alpha-helicity in SDS. While refolding of $\mathrm{SO}_{390}$ was not studied in considerable detail here, kinetic studies of refolding apo-bacterioopsin from SDS into lipid/detergent micelles have enabled detection of multiple folding intermediates in the folding pathway [24,56,57]. In comparison, despite extensive efforts, chemically-denatured rhodopsin, which is characterised by largely disrupted secondary and tertiary structures in the apo-protein state, has thus far resisted refolding [58]. Refolding studies on these three proteins thus suggest that a minimal amount of stabilising structural elements might be required for reversible protein folding. Once again, we 
emphasise that it is very challenging to obtain denatured states of membrane proteins which show minimal aggregation and are amenable to both biophysical characterisation and refolding studies.

\section{Summary}

Using various biophysical techniques, we have studied the denatured states of pSRII in SDS mixed micelles and the kinetics of unfolding and refolding from both the unfolded state with a covalently attached chromophore $\left(\mathrm{SR}_{440}\right)$ and the apo-protein state $\left(\mathrm{SO}_{390}\right)$. Similar to SDS-denatured bacteriorhodopsin, SDS-denatured pSRII retains a high alpha-helical content and loses most of the native retinal-protein contacts. The SDS-denatured states of pSRII, bacteriorhodopsin and bovine rhodopsin have very different refolding properties, even though refolding of bacteriorhodopsin and pSRII both involve predominantly the formation of native tertiary packing. Refolding of SDS-denatured bacteriorhodopsin with bound retinal adheres to two-state folding kinetics; refolding kinetics of the equivalent $\mathrm{SR}_{440}$ state showed clear deviations from the two-state folding regime; and refolding of bovine rhodopsin remains elusive even from partially denatured states.

From this study, we present a low resolution model for the unfolding and folding pathways of pSRII. We propose pSRII as a new model protein for membrane protein folding studies that possibly has a unique folding mechanism, distinct from those of bacteriorhodopsin and bovine rhodopsin. Further high-resolution structural studies of SDS-denatured pSRII will be required to obtain residue-specific information on SDS-protein interactions, and to probe for different species in the denatured states ensemble of pSRII.

\section{Materials and Methods}

\section{Materials:}

SDS (electrophoresis grade) and 50\% (w/v) hydroxylamine hydrochloride were purchased from Sigma-Aldrich. Diheptanoylphosphatidylcholine (c7-DHPC) was purchased from Anatrace.

\section{Protein expression and purification:}


Natronomonas pharaonis sensory rhodopsin II (pSRII) was expressed in E. coli Tuner(DE3)Lacl cells (Novagen) and purified in $50 \mathrm{mM}$ sodium phosphate pH 6.0, $50 \mathrm{mM}$ $\mathrm{NaCl}, 0.05 \%(\mathrm{w} / \mathrm{v})$ sodium azide $\left(\mathrm{NaN}_{3}\right)$ and $2 \%(\mathrm{w} / \mathrm{v})$ c7-DHPC as described previously [59]. Protein concentration was measured using a molar extinction coefficient of $48000 \mathrm{M}^{-1} \mathrm{~cm}^{-1}$ for the native retinal chromophore absorbing at $498 \mathrm{~nm}$, or $49390 \mathrm{M}^{-1} \mathrm{~cm}^{-1}$ for absorbance at $280 \mathrm{~nm}[60]$.

\section{Protein Unfolding:}

pSRII at $\sim 450 \mu \mathrm{M}$ was unfolded by a 67 -fold dilution into unfolding buffer containing $50 \mathrm{mM}$ sodium phosphate $\mathrm{pH} 6.0,50 \mathrm{mM} \mathrm{NaCl}, 0.1 \%$ (w/v) c7-DHPC and different concentrations of denaturants. $\mathrm{NaN}_{3}$ was excluded from unfolding buffers for fair comparison across different biophysical techniques, as the strong UV absorbance by $\mathrm{NaN}_{3}$ interferes with far-UV circular dichroism spectroscopy.

\section{Refolding from $\mathrm{SR}_{440}$ :}

pSRII at $\sim 450 \mu \mathrm{M}$ was unfolded at room temperature for 4 minutes by 33 -fold dilution into unfolding buffer containing $50 \mathrm{mM}$ sodium phosphate $\mathrm{pH} 6.0,50 \mathrm{mM} \mathrm{NaCl}$, and $0.943 X_{\mathrm{SDS}}$ ( $5 \%$ SDS $+0.5 \%$ c7-DHPC). The protein was refolded from the $\mathrm{SR}_{440}$ state by 12 -fold dilution into refolding buffer containing $50 \mathrm{mM}$ sodium phosphate $\mathrm{pH} 6.0,50 \mathrm{mM} \mathrm{NaCl}$, and different $X_{\text {SDS }}$ in $0.5 \%$ c7-DHPC. The quoted $X_{\text {SDS }}$ is the final $X_{\text {SDS }}$ remaining in the reaction after 12-fold dilution of the unfolding reaction into refolding buffer. The refolding reaction was monitored by UV/vis spectroscopy for up to 2 hours.

\section{Refolding from $\mathrm{SO}_{390}$ :}

$\mathrm{SO}_{390}$ was obtained by a 2-hour incubation of pSRII diluted 33-fold into unfolding buffer containing $50 \mathrm{mM}$ sodium phosphate $\mathrm{pH} 6.0,50 \mathrm{mM} \mathrm{NaCl}$, and $0.893 X_{\text {SDS }}(2.5 \%$ SDS + $0.5 \% \mathrm{c} 7$-DHPC). Refolding from $\mathrm{SO}_{390}$ was initiated in a similar manner as for refolding from $\mathrm{SR}_{440}$, and the refolding reaction was monitored by UV/vis spectroscopy for up to 2 hours. The final spectrum of the refolded sample was recorded at least 16 hours after refolding was initiated.

\section{SDS-Polyacrylamide Gel Electrophoresis (SDS-PAGE):}

SDS-PAGE analysis of pSRII at different denaturant concentrations was performed on a $12 \%$ polyacrylamide gel at $90 \mathrm{~V}$ for $15 \mathrm{~min}$ followed by $180 \mathrm{~V}$ for $40 \mathrm{~min}$. $2 \times$ Native loading dye containing $62.5 \mathrm{mM}$ Tris- $\mathrm{HCl}, \mathrm{pH} 6.8,25 \%(\mathrm{v} / \mathrm{v})$ glycerol, $0.01 \%(\mathrm{w} / \mathrm{v})$ bromophenol blue [37] was used for the analysis of aggregation states in unfolded protein samples. PageRuler prestained protein ladder (10-180 kDa) (Thermo Fisher Scientific) was used as molecular 
weight markers. Protein bands were visualised using the Pierce ${ }^{\mathrm{TM}}$ Silver Stain Kit (Thermo Fisher Scientific) according to manufacturer's instructions.

\section{UV/Vis Spectroscopy:}

UV/Vis spectra were recorded at room temperature in a $10 \mathrm{~mm}$ path length cuvette using a UV-1800 spectrophotometer (Shimadzu). Spectra were recorded between 250 and $600 \mathrm{~nm}$ with a fast scan rate (accumulation time $=0.05 \mathrm{~s}$ ) and a sampling interval of $1 \mathrm{~nm}$. Each steady-state spectrum was reported as an average of 3 measurements. Time-course measurements were done by recording spectra in $1 \mathrm{~min}$ or $5 \mathrm{~min}$ intervals using the aforementioned settings, with a typical dead-time of 25 seconds before the first spectrum was recorded.

\section{Far-UV Circular Dichroism (CD) Spectroscopy:}

To avoid strong UV absorbance by $\mathrm{NaN}_{3}, \mathrm{NaN}_{3}$ was first removed from small aliquots $(100 \mu \mathrm{l})$ of pSRII stock solutions by concentrating and diluting 3 times in a $0.5 \mathrm{ml}$ centrifugal filter unit (10-kDa cutoff) (Merck Millipore) using a total of $\sim 1.5 \mathrm{ml}$ of $50 \mathrm{mM}$ sodium phosphate $\mathrm{pH}$ 6.0, $50 \mathrm{mM} \mathrm{NaCl}, 0.1 \%$ (w/v) c7-DHPC. CD spectra were recorded at $25{ }^{\circ} \mathrm{C}$ by scanning between 194 and $250 \mathrm{~nm}$ on an Aviv 410 spectrometer (Aviv Biomedical Inc.) using a 1×1 $\mathrm{mm}$ cuvette. Each sample contains $0.17 \mathrm{mg} / \mathrm{ml}$ protein in a volume of $400 \mu \mathrm{l}$, measured 2 hours after exposure to unfolding buffer. CD spectra of the buffer solutions were subtracted from the sample spectra and smoothed using a window width of 11 and a degree of 2 . Spectra were reported as the average of 3 scans. Data for each experimental condition is reported as the average of 3 independent samples.

All CD spectra were analysed using CDPro [40]. The helicity was estimated by taking basis set 10 containing both soluble and membrane proteins [40]. The built-in secondary structure determining algorithms CONTIN/LL [40,61], SELCON3 [62] and CDSSTR [63] were executed, using delta epsilon as the input.

$$
\Delta \varepsilon=\frac{9}{10 \times 1 \times \times \times n \times 3248}
$$

Here, $\theta$ is the observed ellipticity (millidegrees), I the path length $(\mathrm{cm}), c$ the protein concentration (M) and $n$ the number of peptide bonds, taken as 246 for pSRII.

Molar residue ellipticity (MRE) is calculated as:

$$
\lceil 0\rceil=\Delta \longleftarrow \times 3298
$$


To monitor time-dependent changes in alpha-helical content during SDS-mediated unfolding of pSRII, ellipticity was recorded at fixed wavelengths of $222 \mathrm{~nm}$ and $208 \mathrm{~nm}$ at 30 -second intervals for 2 hours. The typical experimental dead-time is $1 \mathrm{~min}$.

\section{Tryptophan Fluorescence:}

Fluorescence spectra were measured at $25{ }^{\circ} \mathrm{C}$ using a LS55 fluorescence spectrometer (Perkin Elmer) (excitation slit: $5.0 \mathrm{~nm}$, emission slit: $17.5 \mathrm{~nm}$ ). Emission scans were recorded from 310 to $500 \mathrm{~nm}$ using an excitation wavelength of $295 \mathrm{~nm}$, at a scan rate of $100 \mathrm{~nm} / \mathrm{min}$ and with a sampling interval of $0.5 \mathrm{~nm}$. For time-course measurements, fluorescence emission at $335 \mathrm{~nm}$ was recorded with an integration time of $3 \mathrm{~s}$. The typical experimental dead-time was $25 \mathrm{~s}$.

\section{Hydroxylamine treatment of pSRII:}

Hydroxylamine hydrochloride was added to unfolding buffers to a final concentration of 10 $\mathrm{mM}$. The relative concentrations of the monobasic and dibasic sodium phosphate salts were adjusted accordingly to ensure that the final $\mathrm{pH}$ values of all unfolding buffers containing hydroxylamine were consistent (within $0.1 \mathrm{pH}$ unit) with $\mathrm{pH}$ values of matching conditions without hydroxylamine.

\section{Calculations:}

Mole fraction of SDS

The mole fraction of SDS ( $\left.X_{\text {SDS }}\right)$ was calculated as the bulk detergent composition in solution.

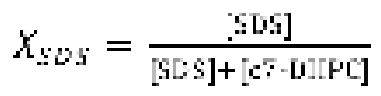

\section{Equilibrium Unfolding}

Absorbance at $498 \mathrm{~nm}$ were plotted against $X_{\mathrm{SDS}}$. Assuming that only the folded and unfolded conformations are present at any given time point of the unfolding reaction, the fraction of protein present in the folded $\left(\delta_{F}\right)$ and unfolded $\left(\delta_{U}\right)$ conformations, respectively, can then be represented as:

$$
f_{\mathrm{P}}+f_{\mathrm{J}}=1
$$

fu was calculated as:

$$
\gamma_{U}=\left(A_{F}-A_{j}\right) /\left(A_{F}-A_{C}\right)
$$

where $A_{5}$ is the $A_{498}$ of completely folded or native protein, $A_{\mathrm{j}}$ is the observed $\mathrm{A}_{498}$ at any point of denaturant concentration, $A_{\mathrm{U}}$ is $\mathrm{A}_{498}$ of the completely denatured or unfolded protein. 


\section{Kinetics fitting:}

UV/vis spectroscopy, CD spectroscopy and tryptophan fluorescence time course data were subjected to Levenberg-Marquardt non-linear least squares fitting using $R$ codes (available upon request).

\section{UV/Vis spectra}

Time courses of absorbance spectra were modelled as the combinations of absorbance peaks varying over time according to a kinetic model. Each peak was treated as a Gaussian peak of the form $e^{-\frac{c^{-}-1^{2}}{2 w^{2}}}$, where $c$ is the wavelength corresponding to the fitted peak centre and $w$ is the half width at half maximum of the fitted Gaussian (Fig. S10c(i)-(iii)).

All absorbance in the region 350-650 $\mathrm{nm}$ was assumed to be due to retinal species varying according to the kinetic model. The kinetic model was described with two consecutive first-order reactions, converting pSRII (absorbing at $498 \mathrm{~nm}$ ) to $\mathrm{SR}_{440}$, which subsequently decays to $\mathrm{SO}_{390}$ :

$$
\operatorname{pSRII~} \stackrel{\dot{k}_{4}}{\rightarrow} \mathrm{SR}_{: 10} \stackrel{\text { int }}{\rightarrow} \mathrm{SO}_{190}
$$

Time courses were unfolded to a three-column matrix with a column for wavelength $(\mathrm{nm})$, time ( $\min$ ), and absorbance for that wavelength at that time. These were then fitted to Equation 6 using the Levenberg-Marquardt least-squares fitting algorithm [64] as implemented in the minpack.LM package [65] for R [66].

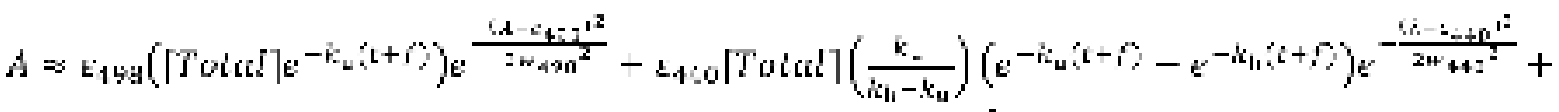

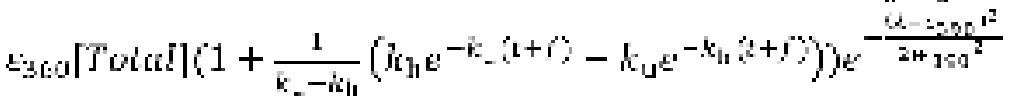

where [Total] is the total protein concentration (see Equation 7), $k_{\mathrm{u}}$ and $k_{\mathrm{h}}$ are the fitted rate constants, and $f$ was an error term that was added to allow for the dead time and the small amount of absorbance at $380 \mathrm{~nm}$ present in native spectra. Rate constants were reported as the mean of three independent measurements, with error bars representing the standard deviation.

As no retinal was added during the experiment, at every point, the Beer-Lambert law was used with a known total concentration to approximate extinction coefficient-like values 
(Equation 7) to convert the amplitudes of the fitted peaks to concentrations while accounting for peak overlap. This allowed peak amplitudes to be replaced in fitting with the concentration terms of the rate equations (Fig. S10c(iv)).

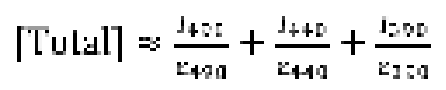

\section{Tryptophan fluorescence and circular dichroism}

Rate equations were used to fit tryptophan fluorescence (intensity at $335 \mathrm{~nm}$ vs. time) and circular dichroism (MRE at $222 \mathrm{~nm}$ vs. time) time courses. Fluorescence increases over time were fit to the sum of the rate equations for $\mathrm{SR}_{440}$ and $\mathrm{SO}_{390}$. The initial fluorescence was subtracted to remove pSRII's contribution and simplify fitting. This yields Equation 8 when $k_{1}$ is sufficiently large.

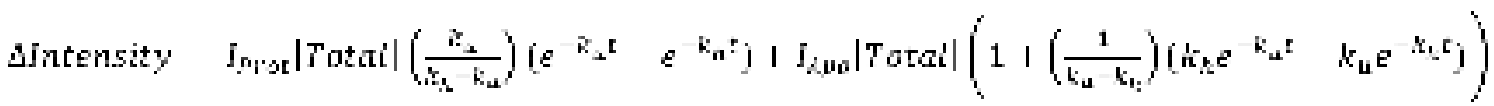

Under conditions where $k_{\mathrm{u}}<k_{\mathrm{h}}$, time courses approximate a single exponential curve. Single and double exponential fits to the data were compared using the Akaike Information Criterion and checked with cross-validation wherever ambiguous (Fig. S10a-b).

Refolding from $\mathrm{SR}_{440}$

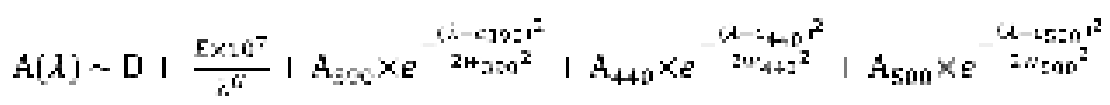

The 350-650 nm region of the absorption spectra of pSRII were fitted to Equation 9. Each time point of the refolding time courses was fitted to a combination of three Gaussian peaks at around $390 \mathrm{~nm}, 440 \mathrm{~nm}$ and $500 \mathrm{~nm}$, and terms for both linear and scattering baseline variation. Changes in the fitted absorbance intensities at $\sim 500 \mathrm{~nm}$ over time were fitted to a double exponential model (Fig. S11d-f):

$$
A_{3}: j^{\sim} A_{1}\left(1-e^{-R_{1} i}\right)+A_{2}\left(e^{-n_{2} i}\right)+c
$$

where $R_{1}$ and $R_{2}$ are the macroscopic rate constants, and $A_{1}$ and $A_{2}$ are the amplitudes of the fast phase and the slow phase, respectively.

$R_{1}$ and $R_{2}$ are the solutions to the following quadratic equation: 


$$
R^{2}-\left(k_{i}+k_{u}+k_{n}\right) R+k_{i} k_{n}=0
$$

Derivation of Equation 11 was done as outlined by Schlebach et al [22].

Based on the quadratic equation, the microscopic rate constants $k_{\mathrm{f}}$ and $k_{\mathrm{u}}$ were extracted using the following relationships and experimentally determined values of $R_{1}, R_{2}$ and $k_{h}$ :

$$
\begin{gathered}
R_{1} k_{2}=k_{1} k_{i} \\
R_{1}-R_{2}=k_{f}+k_{u}+k_{h}
\end{gathered}
$$

Under conditions where the rate of retinal hydrolysis is very slow compared to the rate of folding, changes in the fitted absorbance intensities at $\sim 500 \mathrm{~nm}$ over time were approximated to a single exponential model (Fig. S11a-c):

$$
A_{3}: j^{\sim} \sim A_{1}\left(1-e^{-k ! t}\right)+c
$$

where the observed rate constant was approximated as the de novo refolding rate $k_{\mathrm{f}}$.

\section{Refolding from $\mathrm{SO}_{390}$}

The absorption spectrum of native pSRII contains one main retinal absorption peak at 498 $\mathrm{nm}, 3$ shoulder peaks at $460 \mathrm{~nm}, 420 \mathrm{~nm}$, and $370 \mathrm{~nm}$, and protein absorption peak at 280 $\mathrm{nm}$ [33]. Five native spectra were fitted to these peaks, four of which were symmetrical Gaussian functions, and the $420 \mathrm{~nm}$ peak, the width of which was allowed to vary sigmoidally with wavelength to allow for previously reported skewedness [33]. Retinal peak amplitudes were normalised to have a sum of 1 , and parameters were averaged for the retinal peaks to find a peak profile for native pSRII.

Each time point of the refolding time courses was fitted as a combination of some multiple of the native profile, a peak around $390 \mathrm{~nm}$ for free retinal and terms for both linear and scattering baseline variation (see the first two terms of Equation 15):

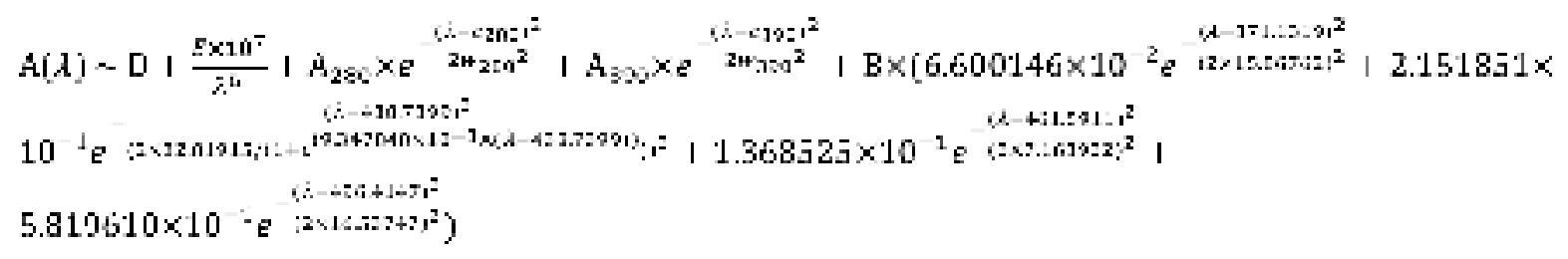


The concentrations of free retinal and native pSRII were extracted from these timepoints. Since the total concentration is constant, and the parameters $B$ and $A_{390}$ were assumed to be proportional to the concentrations of native pSRII and free retinal respectively, the concentrations of these species were estimated by fitting these parameters for each timepoint to Equation 16.

$$
[\text { Total }]=\mathrm{A}_{330} \times \mathrm{SO}_{390}+\mathrm{B} \times \mathrm{pSRII}
$$

where [Total] is the total protein concentration determined from the $A_{280}$ peak, $A_{390}$ and $B$ were fitted values from Equation 15, and $\mathrm{SO}_{390}$ and pSRII were fitted coefficients, later used to calculate concentrations.

The estimated values for $\mathrm{SO}_{390}$ concentration were fitted to an exponential decay model of the form $A \times e^{-k_{u ; v} t}+C$, from which an apparent rate, $k_{\mathrm{app}}$, and an asymptote, $\mathrm{C}$, were obtained (Fig. S12). The asymptote of the curve estimates the fraction of the total concentration that cannot be refolded on the time scale of the experiment ( 2 hours).

\section{Acknowledgements}

This work was funded in part through a BBSRC research grant to D.N. (BB/K01983 X/1) and by an International Incoming Fellowship of the PF7 Marie Curie Actions (to J.K.S.). Y.L.T. is the grateful recipient of a Cambridge Trust Scholarship. We would like to thank Prof. Laura Itzhaki (University of Cambridge) for helpful discussions on protein folding.

\section{References}

[1] M. Uhlen, L. Fagerberg, B.M. Hallstrom, C. Lindskog, P. Oksvold, A. Mardinoglu, A. Sivertsson, C. Kampf, E. Sjostedt, A. Asplund, I. Olsson, K. Edlund, E. Lundberg, S. Navani, C.A.-K. Szigyarto, J. Odeberg, D. Djureinovic, J.O. Takanen, S. Hober, T. Alm, P.-H. Edqvist, H. Berling, H. Tegel, J. Mulder, J. Rockberg, P. Nilsson, J.M. Schwenk, M. Hamsten, K. von Feilitzen, M. Forsberg, L. Persson, F. Johansson, M. Zwahlen, G. von Heijne, J. Nielsen, F. Ponten, Tissue-based map of the human proteome, Science (80-. ). 347 (2015) 1260419-1260419. doi:10.1126/science.1260419.

[2] N.G. Bednarska, J. Schymkowitz, F. Rousseau, J. Van Eldere, Protein aggregation in bacteria: The thin boundary between functionality and toxicity, Microbiol. (United Kingdom). 159 (2013) 1795-1806. doi:10.1099/mic.0.069575-0. 
[3] R. V. Rao, D.E. Bredesen, Misfolded proteins, endoplasmic reticulum stress and neurodegeneration, Curr. Opin. Cell Biol. 16 (2004) 653-662.

doi:10.1016/j.ceb.2004.09.012.

[4] A. Ulloa-Aguirre, P. Michael Conn, Pharmacoperones: a new therapeutic approach for diseases caused by misfolded $\mathrm{G}$ protein-coupled receptors., Recent Pat. Endocr. Metab. Immune Drug Discov. 5 (2011) 13-24. doi:10.2174/187221411794351851.

[5] A.I. Bartlett, S.E. Radford, An expanding arsenal of experimental methods yields an explosion of insights into protein folding mechanisms, Nat. Struct. Mol. Biol. 16 (2009) 582-588. doi:10.1038/nsmb.1592.

[6] J.U. Bowie, Solving the membrane protein folding problem, Nature. 438 (2005) $581-$ 589. doi:10.1038/nature04395.

[7] A.M. Stanley, K.G. Fleming, The process of folding proteins into membranes: Challenges and progress, Arch. Biochem. Biophys. 469 (2008) 46-66. doi:10.1016/j.abb.2007.09.024.

[8] K.S. Huang, H. Bayley, M.J. Liao, E. London, H.G. Khorana, Refolding of an integral membrane protein. Denaturation, renaturation, and reconstitution of intact bacteriorhodopsin and two proteolytic fragments., J. Biol. Chem. 256 (1981) 38023809.

[9] J.K. Nagy, W.L. Lonzer, C.R. Sanders, Kinetic study of folding and misfolding of diacylglycerol kinase in model membranes, Biochemistry. 40 (2001) 8971-8980. doi:10.1021/bi010202n.

[10] D.E. Otzen, Folding of DsbB in mixed micelles: a kinetic analysis of the stability of a bacterial membrane protein., J. Mol. Biol. 330 (2003) 641-649. doi:10.1016/S00222836(03)00624-7.

[11] N. Di Bartolo, E.L.R. Compton, T. Warne, P.C. Edwards, C.G. Tate, G.F.X. Schertler, P.J. Booth, Complete reversible refolding of a G-protein coupled receptor on a solid support, PLoS One. 11 (2016). doi:10.1371/journal.pone.0151582.

[12] M.R. Sanders, H.E. Findlay, P.J. Booth, Lipid bilayer composition modulates the unfolding free energy of a knotted $\alpha$-helical membrane protein, Proc. Natl. Acad. Sci. 115 (2018) E1799-E1808. doi:10.1073/pnas.1714668115.

[13] F.W. Lau, J.U. Bowie, A method for assessing the stability of a membrane protein, Biochemistry. 36 (1997) 5884-5892. doi:10.1021/bi963095j.

[14] F.N. Barrera, M.L. Renart, M.L. Molina, J.A. Poveda, J.A. Encinar, A.M. Fernández, J.L. Neira, J.M. González-Ros, Unfolding and refolding in vitro of a tetrameric, $\alpha-$ helical membrane protein: The prokaryotic potassium channel KcsA, Biochemistry. 44 (2005) 14344-14352. doi:10.1021/bi050845t.

[15] H.E. Findlay, N.G. Rutherford, P.J.F. Henderson, P.J. Booth, Unfolding free energy of a two-domain transmembrane sugar transport protein, Proc. Natl. Acad. Sci. 107 (2010) 18451-18456. doi:10.1073/pnas.1005729107.

[16] D. Miller, K. Charalambous, D. Rotem, S. Schuldiner, P. Curnow, P.J. Booth, In vitro Unfolding and Refolding of the Small Multidrug Transporter EmrE, J. Mol. Biol. 393 (2009) 815-832. doi:10.1016/j.jmb.2009.08.039.

[17] E.A. Roman, J.M. Argüello, F.L. González Flecha, Reversible Unfolding of a Thermophilic Membrane Protein in Phospholipid/Detergent Mixed Micelles, J. Mol. Biol. 397 (2010) 550-559. doi:10.1016/j.jmb.2010.01.045.

[18] E. London, H.G. Khorana, Denaturation and renaturation of bacteriorhodopsin in detergents and lipid-detergent mixtures., J. Biol. Chem. 257 (1982) 7003-7011.

[19] C. Yang, R. Horn, H. Paulsen, The light-harvesting chlorophyll a/b complex can be reconstituted in vitro from its completely unfolded apoprotein, Biochemistry. 42 (2003) 4527-4533. doi:10.1021/bi0273157.

[20] P. Curnow, P.J. Booth, Combined kinetic and thermodynamic analysis of $\alpha$-helical membrane protein unfolding, Proc. Natl. Acad. Sci. 104 (2007) 18970-18975. doi:10.1073/pnas.0705067104.

[21] P. Curnow, P.J. Booth, The transition state for integral membrane protein folding., Proc. Natl. Acad. Sci. U. S. A. 106 (2009) 773-778. doi:10.1073/pnas.0806953106. 
[22] J.P. Schlebach, N.B. Woodall, J.U. Bowie, C. Park, Bacteriorhodopsin folds through a poorly organized transition state, J. Am. Chem. Soc. 136 (2014) 16574-16581. doi:10.1021/ja508359n.

[23] D.E. Otzen, Protein Unfolding in Detergents: Effect of Micelle Structure, lonic Strength, pH, and Temperature, Biophys. J. 83 (2002) 2219-2230. doi:10.1016/S00063495(02)73982-9.

[24] P.J. Booth, S.L. Flitsch, L.J. Stern, D.A. Greenhalgh, P.S. Kim, H.G. Khorana, Intermediates in the folding of the membrane protein bacteriorhodopsin, Nat. Struct. Biol. 2 (1995) 139-143. doi:10.1038/nsb0295-139.

[25] J.L. Popot, D.M. Engelman, Membrane Protein Folding and Oligomerization: The Two-Stage Model, Biochemistry. 29 (1990) 4031-4037. doi:10.1021/bi00469a001.

[26] J.F. Hunt, T.N. Earnest, O. Bousche, K. Kalghatgi, K. Reilly, C. Horvath, K.J. Rothschild, D.M. Engelman, A biophysical study of integral membrane protein folding, Biochemistry. 36 (1997) 15156-15176. doi:10.1021/bi970146jlrbi970146j [pii].

[27] A. Dutta, T.Y. Kim, M. Moeller, J. Wu, U. Alexiev, J. Klein-Seetharaman, Characterization of membrane protein non-native states. 2. the SDS-unfolded states of rhodopsin, Biochemistry. 49 (2010) 6329-6340. doi:10.1021/bi100339x.

[28] K.D. Ridge, S.S. Lee, L.L. Yao, In vivo assembly of rhodopsin from expressed polypeptide fragments., Proc. Natl. Acad. Sci. U. S. A. 92 (1995) 3204-3208. doi:10.1073/pnas.92.8.3204.

[29] K.T. Sapra, P.S.H. Park, K. Palczewski, D.J. Muller, Mechanical properties of bovine rhodopsin and bacteriorhodopsin: Possible roles in folding and function, Langmuir. 24 (2008) 1330-1337. doi:10.1021/la702299z.

[30] A. Dutta, C. Altenbach, S. Mangahas, N. Yanamala, E. Gardner, W.L. Hubbell, J. Klein-Seetharaman, Differential dynamics of extracellular and cytoplasmic domains in denatured states of rhodopsin, Biochemistry. 53 (2014) 7160-7169. doi:10.1021/bi401557e.

[31] O. Tastan, E. Yu, M. Ganapathiraju, A. Aref, a J. Rader, J. Klein-Seetharaman, Comparison of stability predictions and simulated unfolding of rhodopsin structures., Photochem. Photobiol. 83 (2007) 351-362. doi:10.1562/2006-06-20-RA-942.

[32] J. Klein-Seetharaman, Dual role of interactions between membranous and soluble portions of helical membrane receptors for folding and signaling, Trends Pharmacol. Sci. 26 (2005) 183-189. doi:10.1016/j.tips.2005.02.009.

[33] I. Chizhov, G. Schmies, R. Seidel, J.R. Sydor, B. Luttenberg, M. Engelhard, The photophobic receptor from Natronobacterium pharaonis: temperature and $\mathrm{pH}$ dependencies of the photocycle of sensory rhodopsin II, Biophys. J. 75 (1998) 9991009. doi:10.1016/s0006-3495(98)77588-5.

[34] B. Honig, a D. Greenberg, U. Dinur, T.G. Ebrey, Visual-pigment spectra: implications of the protonation of the retinal Schiff base., Biochemistry. 15 (1976) 4593-9. doi:10.1021/bi00666a008.

[35] J. Horwitz, J. Heller, Interactions of All-trans-, 9-, 11-, and 13-cis-retinal, All-transretinyl Acetate, and Retinoic Acid with Human Retinol-binding Protein and Prealbumin, J. Biol. Chem. 248 (1973) 6317-6324. http://www.jbc.org.ezproxy.library.wisc.edu/content/248/18/6317.

[36] G.Q. Chen, E. Gouaux, Probing the folding and unfolding of wild-type and mutant forms of bacteriorhodopsin in micellar solutions: Evaluation of reversible unfolding conditions, Biochemistry. 38 (1999) 15380-15387. doi:10.1021/bi9909039.

[37] A. Dutta, K.C. Tirupula, U. Alexiev, J. Klein-Seetharaman, Characterization of membrane protein non-native states. 1. Extent of unfolding and aggregation of rhodopsin in the presence of chemical denaturants, Biochemistry. 49 (2010) 63176328. doi:10.1021/bi100338e.

[38] Y. Yu, Characterisation of the membrane protein complex formed by Natronomonas pharaonis sensory rhodopsin pSRII and its cognate transducer pHtrll (Ph.D. thesis), University of Cambridge, 2015.

[39] K. Takeda, Y. Moriyama, Circular dichroism studies on helical structure preferences of 
amino acid residues of proteins caused by sodium dodecyl sulfate., J. Protein Chem. 9 (1990) 573-82. http://www.ncbi.nlm.nih.gov/pubmed/2085382.

[40] N. Sreerama, R.W. Woody, Estimation of protein secondary structure from circular dichroism spectra: comparison of CONTIN, SELCON, and CDSSTR methods with an expanded reference set., Anal. Biochem. 287 (2000) 252-260.

doi:10.1006/abio.2000.4880.

[41] S. Faham, D. Yang, E. Bare, S. Yohannan, J.P. Whitelegge, J.U. Bowie, Side-chain contributions to membrane protein structure and stability, J Mol Biol. 335 (2004) 297305. doi:S0022283603013196 [pii].

[42] P.E. Blatz, J.H. Mohler, H. V Navangul, Anion-induced wavelength regulation of absorption maxima of Schiff bases of retinal, Biochemistry. 11 (1972) 848-855. doi:10.1021/bi00755a026.

[43] T.G. Ebrey, Energy Transfer in Rhodopsin, N-Retinyl-Opsin, and Rod Outer Segments, Proc. Natl. Acad. Sci. U. S. A. 68 (1971) 713-716. doi:10.1073/pnas.68.4.713.

[44] R.G. Adams, D. Kennedy, V.J. Wulff, H. V. Zonana, Rhodopsin bleaching in the presence of hydroxylamine, Arch. Biochem. Biophys. 75 (1958) 534-536. doi:10.1016/0003-9861(58)90451-X.

[45] S. Subramaniam, T. Marti, S.J. Rosselet, K.J. Rothschild, H.G. Khorana, The reaction of hydroxylamine with bacteriorhodopsin studied with mutants that have altered photocycles: selective reactivity of different photointermediates, Proc Natl Acad Sci U S A. 88 (1991) 2583-2587.

http://www.ncbi.nlm.nih.gov/entrez/query.fcgi?cmd=Retrieve\&db=PubMed\&dopt=Citat ion\&list_uids=2006195\%5Cnhttp://www.pnas.org/content/88/6/2583.full.pdf.

[46] U. Zadok, J.P. Klare, M. Engelhard, M. Sheves, The hydroxylamine reaction of sensory rhodopsin II: light-induced conformational alterations with $\mathrm{C} 13=\mathrm{C} 14$ nonisomerizable pigment, Biophys J. 89 (2005) 2610-2617.

doi:10.1529/biophysj.105.065631.

[47] B. Becher, J.Y. Cassim, Effects of Bleaching and Regeneration on the Purple Membrane Structure of Halobacterium halobium, Biophys. J. 19 (1977) 285-297. doi:10.1016/S0006-3495(77)85588-4.

[48] C.N. Rafferty, J.Y. Cassim, D.G. McConnell, Circular dichroism, optical rotatory dispersion, and absorption studies on the conformation of bovine rhodopsin in situ and solubilized with detergent, Biophys. Struct. Mech. 2 (1977) 277-320. doi:10.1007/BF00537501.

[49] Y. Croonen, E. Gelade, M. Van Der Zegel, H. M. Van der Auweraer, Vandendriessche, F.C. De Schryver, M. Almgren, Influence of Salt, Detergent Concentration, and Temperature on the Fluorescence Quenching of I-Methylpyrene in Sodium Dodecyl Sulfate with m-Dicyanobenzene, J. Phys. Chem. 87 (1983) 1426-1431.

[50] J.H. Clint, Surfactant Aggregation, Surfactant Aggreg. 61 (1992) 271. doi:10.1007/978-94-011-2272-6.

[51] J.P. Schlebach, Z. Cao, J.U. Bowie, C. Park, Revisiting the folding kinetics of bacteriorhodopsin, Protein Sci. 21 (2012) 97-106. doi:10.1002/pro.766.

[52] E.Y. Chi, S. Krishnan, T.W. Randolph, J.F. Carpenter, Physical stability of proteins in aqueous solution: Mechanism and driving forces in nonnative protein aggregation, Pharm. Res. 20 (2003) 1325-1336. doi:10.1023/A:1025771421906.

[53] H. Hamada, T. Arakawa, K. Shiraki, Effect of additives on protein aggregation., Curr. Pharm. Biotechnol. 10 (2009) 400-407. doi:10.2174/138920109788488941.

[54] M. Lebendiker, T. Danieli, Production of prone-to-aggregate proteins, FEBS Lett. 588 (2014) 236-246. doi:10.1016/j.febslet.2013.10.044.

[55] S. Kawamura, S. Yokoyama, Functional characterization of visual and nonvisual pigments of American chameleon (Anolis carolinensis), Vision Res. 38 (1998) 37-44. doi:10.1016/S0042-6989(97)00160-0.

[56] P.J. Booth, A. Farooq, S.L. Flitsch, Retinal binding during folding and assembly of the membrane protein bacteriorhodopsin, Biochemistry. 35 (1996) 5902-5909. 
doi:10.1021/bi960129e.

[57] M.L. Riley, B.A. Wallace, S.L. Flitsch, P.J. Booth, Slow a helix formation during folding of a membrane protein, Biochemistry. 36 (1997) 192-196. doi:10.1021/bi962199r.

[58] O. Tastan, A. Dutta, P. Booth, J. Klein-Seetharaman, Retinal proteins as model systems for membrane protein folding, Biochim. Biophys. Acta - Bioenerg. 1837 (2014) 656-663. doi:10.1016/j.bbabio.2013.11.021.

[59] A. Gautier, H.R. Mott, M.J. Bostock, J.P. Kirkpatrick, D. Nietlispach, Structure determination of the seven-helix transmembrane receptor sensory rhodopsin II by solution NMR spectroscopy., Nat. Struct. Mol. Biol. 17 (2010) 768-774. doi:10.1038/nsmb.1807.

[60] B. Scharf, B. Hess, M. Engelhard, Chromophore of sensory rhodopsin II from Halobacterium halobium., Biochemistry. 31 (1992) 12486-92. doi:10.1021/bi00065a045.

[61] S.W. Provencher, J. Glockner, Estimation of globular protein secondary structure from circular dichroism, Biochemistry. 20 (1981) 33-37. doi:10.1021/bi00504a006.

[62] N. Sreerama, R.W. Woody, A Self-Consistent Method for the Analysis of Protein Secondary Structure from Circular Dichroism, Anal. Biochem. 209 (1993) 32-44. doi:10.1006/abio.1993.1079.

[63] P. Manavalan, W.C. Johnson, Variable selection method improves the prediction of protein secondary structure from circular dichroism spectra, Anal. Biochem. 167 (1987) 76-85. doi:10.1016/0003-2697(87)90135-7.

[64] D.W. Marquardt, An Algorithm for Least-Squares Estimation of Nonlinear Parameters, J. Soc. Ind. Appl. Math. 11 (1963) 431-441. doi:10.1137/0111030.

[65] T. V Elzhov, K.M. Mullen, A.-N. Spiess, B. Bolker, Package "minpack.Im": R Interface to the Levenberg-Marquardt Nonlinear Least-Squares Algorithm Found in MINPACK, Plus Support for Bounds, 2016. https://cran.rproject.org/web/packages/minpack.Im/minpack.Im.pdf.

[66] R Development Core Team, R: A Language and Environment for Statistical Computing, 2011. doi:10.1007/978-3-540-74686-7. 


\begin{tabular}{|c|cc|}
\hline \multirow{2}{*}{$\boldsymbol{X}_{\mathrm{SDS}}$} & \multicolumn{2}{|c|}{ Rate constants $\left(\times 1 \mathbf{1 0}^{-4} \mathbf{s}^{-1}\right)$} \\
\cline { 2 - 3 } & $\boldsymbol{k}_{\mathrm{u}}$ & $\boldsymbol{k}_{\mathrm{h}}$ \\
\hline 0.834 & $18 \pm 3$ & $3.7 \pm 0.2$ \\
0.870 & $36 \pm 11$ & $3.9 \pm 0.3$ \\
0.893 & $44 \pm 9$ & $4.5 \pm 0.3$ \\
0.909 & $62 \pm 12$ & $3.8 \pm 0.4$ \\
0.930 & $73 \pm 4$ & $4.1 \pm 0.9$ \\
0.943 & $106 \pm 8$ & $4.2 \pm 0.9$ \\
0.962 & $98 \pm 17$ & $4.5 \pm 0.8$ \\
0.971 & $111 \pm 24$ & $4.4 \pm 0.6$ \\
\hline
\end{tabular}

Table 1. Rate constants for pSRII unfolding in SDS/c7-DHPC mixed micelles. $k_{\mathrm{u}}$ is the first-order rate constant for the formation of $\mathrm{SR}_{440}$ and $k_{\mathrm{h}}$ is the rate constant for Schiff base hydrolysis. Errors are reported as standard deviations of 3 independent measurements.

\begin{tabular}{|c|cccc|}
\hline \multirow{2}{*}{$\boldsymbol{X}_{\mathrm{SDS}}$} & \multicolumn{4}{|c|}{ Rate constants $\times \mathbf{1 0}^{-4}\left(\mathbf{s}^{-1}\right)$} \\
\cline { 2 - 5 } & $\mathbf{R}_{\mathbf{1}}$ & $\mathbf{R}_{\mathbf{2}}$ & $\boldsymbol{k}_{\boldsymbol{f}}$ & $\boldsymbol{k}_{\boldsymbol{u}}$ \\
\hline 0.698 & - & - & $199 \pm 52$ & - \\
0.723 & - & - & $110 \pm 10$ & - \\
0.745 & $81 \pm 18$ & $0.16 \pm 0.05$ & $74 \pm 18$ & $3.5 \pm 1.4$ \\
0.755 & $46 \pm 7$ & $0.32 \pm 0.01$ & $39 \pm 7$ & $4.0 \pm 0.6$ \\
0.763 & $31 \pm 10$ & $0.41 \pm 0.18$ & $25 \pm 10$ & $3.3 \pm 1.8$ \\
0.772 & $23 \pm 3$ & $0.76 \pm 0.09$ & $15 \pm 3$ & $4.7 \pm 0.8$ \\
0.779 & $19 \pm 5$ & $0.87 \pm 0.62$ & $12 \pm 6$ & $3.9 \pm 2.9$ \\
\hline
\end{tabular}

Table 2. Rate constants extracted for refolding pSRII from $\mathbf{S R}_{440}$. Under conditions where the rate of retinal hydrolysis is very slow compared to the rate of folding $(0.698-0.723$ $X_{\mathrm{SDS}}$ ), changes in the fitted absorbance at $\sim 500 \mathrm{~nm}$ over time were approximated to a single exponential model (Equation 14). In higher $X_{\mathrm{SDS}}$, changes in the fitted absorbance at $\sim 500$ $\mathrm{nm}$ over time were fitted to a double exponential model to yield the macroscopic rate constants $\mathrm{R}_{1}$ and $\mathrm{R}_{2}$ (Equation 10). The microscopic rate constants $k_{\mathrm{f}}$ and $k_{\mathrm{u}}$ were extracted using experimentally determined values of $R_{1}, R_{2}$ and $k_{h}$ (Equations 11-13). Errors are reported as standard deviations of 3 independent measurements.

\begin{tabular}{|c|c|}
\hline $\boldsymbol{X}_{\mathrm{SDS}}$ & $\boldsymbol{k}_{\mathrm{app}} \times 10^{-4}\left(\mathbf{s}^{-1}\right)$ \\
\hline 0.410 & $12.9 \pm 2.0$ \\
\hline 0.436 & $5.1 \pm 1.5$ \\
\hline 0.459 & $4.6 \pm 1.2$ \\
\hline 0.480 & $3.0 \pm 0.7$ \\
\hline 0.500 & $2.0 \pm 0.6$ \\
\hline 0.519 & $1.7 \pm 0.2$ \\
\hline
\end{tabular}

Table 3. Apparent rate constants $\left(k_{\mathrm{app}}\right)$ for refolding $\mathrm{pSRII}$ from $\mathbf{S O}_{390} . k_{\text {app }}$ takes into account both retinal re-attachment (reformation of the Schiff base) and protein folding. Errors are reported as standard deviations of 3 independent measurements. 


\section{Figure Legends}

Figure 1. Denaturant screening for unfolding pSRII. (a) Retinal binding pocket of pSRII, where the all-trans retinal chromophore is covalently linked to Lys-205 via a protonated Schiff base. (b) Changes in the UV/vis absorbance of the retinal chromophore during denaturation of pSRII. The insets show schematic representations of the binding pocket and the corresponding photos of $90 \mu \mathrm{M}$ pSRII in different spectral states. (c-e) Loss of retinal binding, determined by monitoring changes to the retinal absorbance peak by UV/Vis spectroscopy, is taken as the hallmark of pSRII unfolding. All spectra are scaled to the 280 $\mathrm{nm}$ peak of the native spectrum (red). Only the 300-600 $\mathrm{nm}$ spectral range is displayed to illustrate changes in the retinal absorbance peak. pSRII was exposed to denaturation for 1 day in water-soluble denaturants (c), anionic and cationic detergents (d), and zwitterionic and non-ionic detergents (e). Only SDS, an anionic detergent, led to complete loss of native retinal-protein contacts $\left(\lambda_{\max }=390 \mathrm{~nm}\right)$.

Figure 2. Detection of aggregates for pSRII unfolded in SDS + 0.1\% c7-DHPC at pH 6.0. Silver-stained $12 \%$ SDS-PAGE gel showing increased aggregation over prolonged unfolding time of pSRII in $0 \%$ (lanes 2-5), $0.5 \%$ (lanes 6-9) and 3\% SDS (lanes 10-13) at pH 6.0 for 0 min, $2 \mathrm{~h}, 24 \mathrm{~h}$ and $72 \mathrm{~h}$ at $25^{\circ} \mathrm{C}$. Lanes 1 and 14, molecular weight marker; lane 15, pSRII boiled in $3 \%$ SDS $+9 \mathrm{M}$ urea (3S9U) for 5 minutes at $95^{\circ} \mathrm{C}$.

Figure 3. Secondary structure changes in SDS-denatured pSRII at pH 6.0. (a) CD spectra and (b) MRE at $222 \mathrm{~nm}$ for $7 \mu \mathrm{M}(0.2 \mathrm{mg} / \mathrm{ml})$ pSRII in $0-0.998 X_{\text {SDs }}(0-30 \%$ SDS in $0.1 \%$ c7-DHPC). Inset in panel (b) shows expanded view for the $0.75-1.00 X_{\text {SDs }}$ region. Error bars represent the standard deviation of 3 independent measurements.

Figure 4. Loosening of the retinal binding pocket in SDS-denatured pSRII at pH 6.0. (a) Time-resolved UV/vis spectra of pSRII unfolded in $0.980 X_{\text {SDS }}$ (3\% SDS in $0.1 \%$ c7-DHPC). (b) SDS-induced equilibrium unfolding of pSRII in the presence of $0.5 \%$ c7-DHPC monitored by UV/vis spectroscopy. $\lambda_{\max }$ of the retinal chromophore at $t=0 \mathrm{~min}$, obtained by nonlinear least squares fitting, is also plotted against $X_{\text {SDS }}$, showing that the chromophore $\lambda_{\max }$ is dependent on $X_{\text {SDS }}$. Error bars represent the standard deviation of 3 independent measurements.

Figure 5. Tryptophan fluorescence of SDS-denatured pSRII at pH 6.0. (a) Solution-state NMR structure of pSRII (PDB 2KSY), with tryptophan residues shown as pink spheres. (b) Tryptophan fluorescence emission spectra of $7 \mu \mathrm{M}$ pSRII unfolded for 2 hours in 0-0.998 $X_{\text {SDS }}\left(0-30 \%\right.$ SDS in $0.1 \%$ c7-DHPC). Emission $\lambda_{\max }(337 \mathrm{~nm})$ of $p S R I I$ in $0 X_{\text {SDS }}$ is shown as dotted line.

Figure 6. Assessing solvent accessibility of the retinal binding pocket using hydroxylamine at pH 6.0. (a) Reaction mechanism of hydroxylamine with pSRII protonated Schiff base to form retinal oxime. Structures were drawn using MolView. (b) Time-resolved UV/vis spectra of pSRII unfolded in (i) $10 \mathrm{mM}$ hydroxylamine $\left(0 X_{\mathrm{SDS}}\right)$ and (ii) $0.980 X_{\mathrm{SDS}}$ (3\% SDS) $+10 \mathrm{mM}$ hydroxylamine in $0.1 \%$ c7-DHPC. (c) Emission spectra of $7 \mu \mathrm{M}$ pSRII unfolded for 2 hours in $0-0.998 X_{\mathrm{SDS}}+10 \mathrm{mM}$ hydroxylamine in $0.1 \% \mathrm{c} 7-\mathrm{DHPC}$. Emission $\lambda_{\max }(338.5 \mathrm{~nm})$ of pSRII in $0 X_{\text {SDS }}+10 \mathrm{mM}$ hydroxylamine is shown as dotted line. (d) MRE at $222 \mathrm{~nm}$ for pSRII in 0-0.998 $X_{\mathrm{SDS}}+10 \mathrm{mM}$ hydroxylamine. Inset shows expanded view for the $0.75-1.00 X_{S D S}$ region. Error bars represent the standard deviation of 3 independent measurements.

Figure 7. Refolding of pSRII from the unfolded state $\left(\mathbf{S R}_{\mathbf{4 4 0}}\right)$. (a) Absorption spectra for refolding from $\mathrm{SR}_{440}$ in (i) $0.698 X_{\mathrm{SDS}}$ and (ii) $0.779 X_{\mathrm{SDS}}$, both in $0.5 \% \mathrm{c} 7-\mathrm{DHPC}$. Both regeneration of native chromophore $(498 \mathrm{~nm})$ and formation of $\mathrm{SO}_{390}$ can be observed, with the rate of refolding being dependent on $X_{\text {SDS }}$. (b) Changes in absorbance at $\sim 500 \mathrm{~nm}$ in 
high $X_{\text {SDS }}$ can be fitted to a double exponential to yield two macroscopic rate constants, $R_{1}$ and $R_{2}$. (c) Chevron plot summarising the extracted microscopic unfolding and refolding rates, $k_{\mathrm{u}}$ and $k_{\mathrm{f}}$. Error bars in panels (b) and (c) represent the standard deviation of 3 independent measurements.

Figure 8. Refolding of pSRII from the apo-protein state $\left(\mathbf{S O}_{390}\right)$. (a) Absorption spectra for refolding from $\mathrm{SO}_{390}$ in (i) $0.410 X_{\mathrm{SDS}}$ and (ii) $0.519 X_{\mathrm{SDS}}$, both in $0.5 \% \mathrm{c} 7$-DHPC. (b) Natural logarithm of the apparent refolding rate (In $k_{\text {app }}$ ) from $\mathrm{SO}_{390}$ is linearly dependent on $X_{\text {SDS. }}$ (c) Refolding yield of $\mathrm{SO}_{390}$ decreases with increasing amount of time spent in the unfolded state, suggesting that only the monomeric species might be capable of rebinding retinal and regenerating the native chromophore. Error bars in panels (b) and (c) represent the standard deviation of 3 independent measurements. Results from one representative gel is shown for estimating the monomeric content by SDS-PAGE (see Fig. S1d). 
(a)

(b)
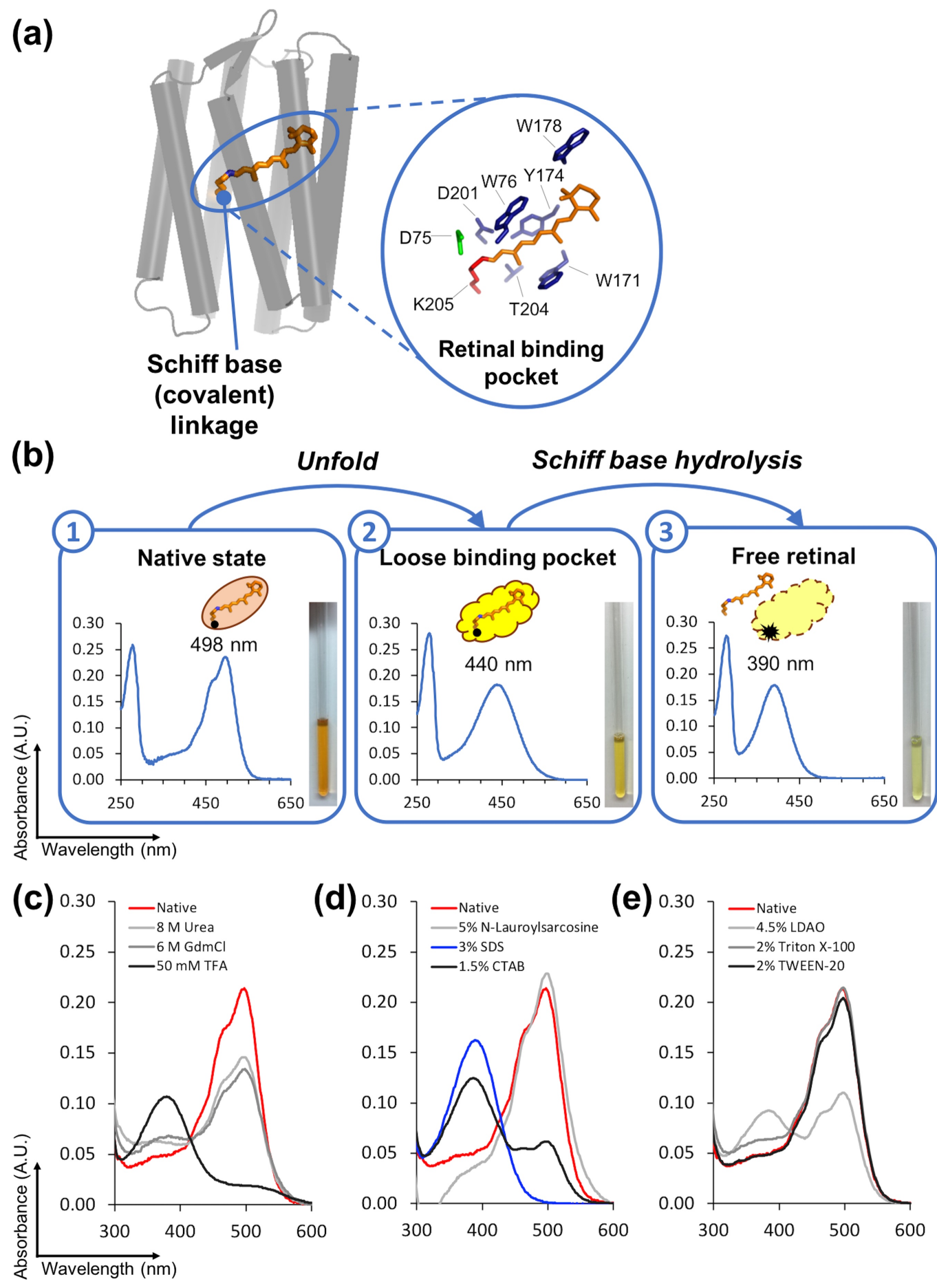
[SDS] (\%)
Time (h) $\quad M \frac{0 \% \text { SDS }}{022472} \frac{0.5 \% \text { SDS }}{022472} \frac{3 \% \text { SDS }}{022472} M_{3} S^{9}$

(kDa) 140

80

65

50

40

30

25

15

10 

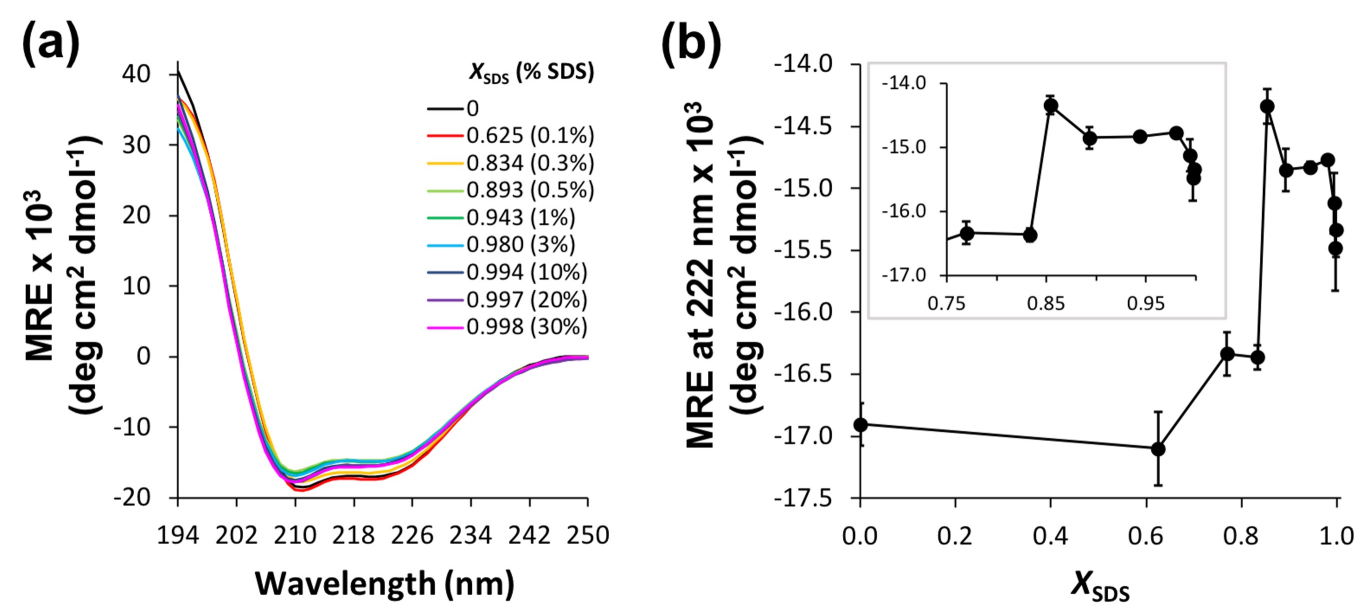


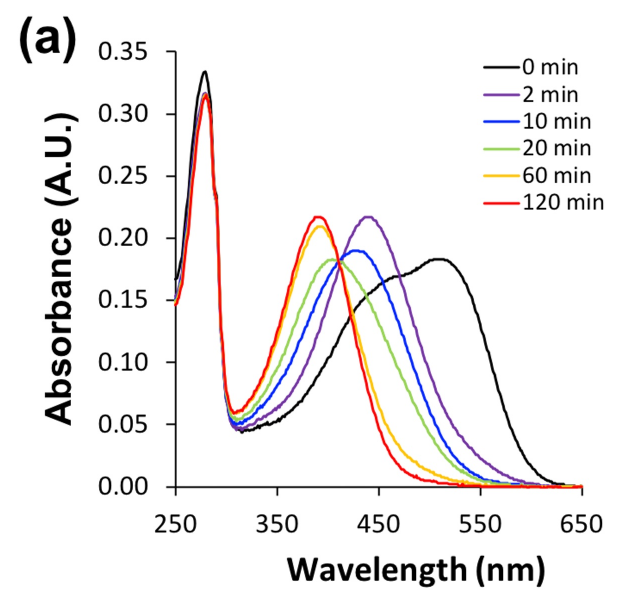

(b)

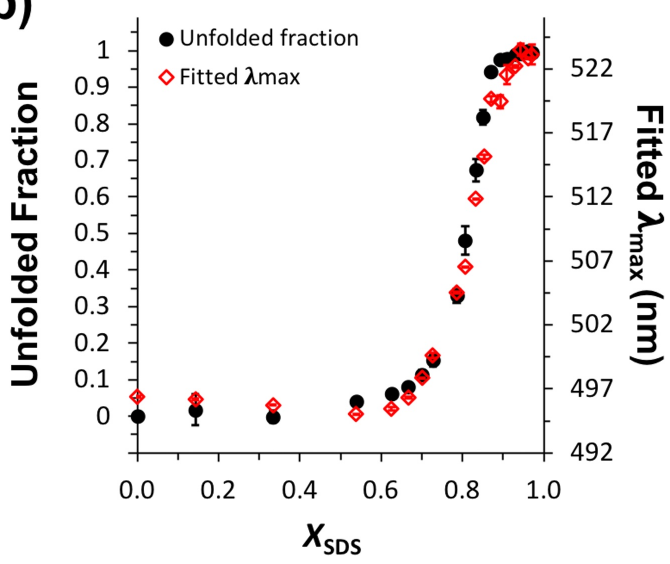


(a)

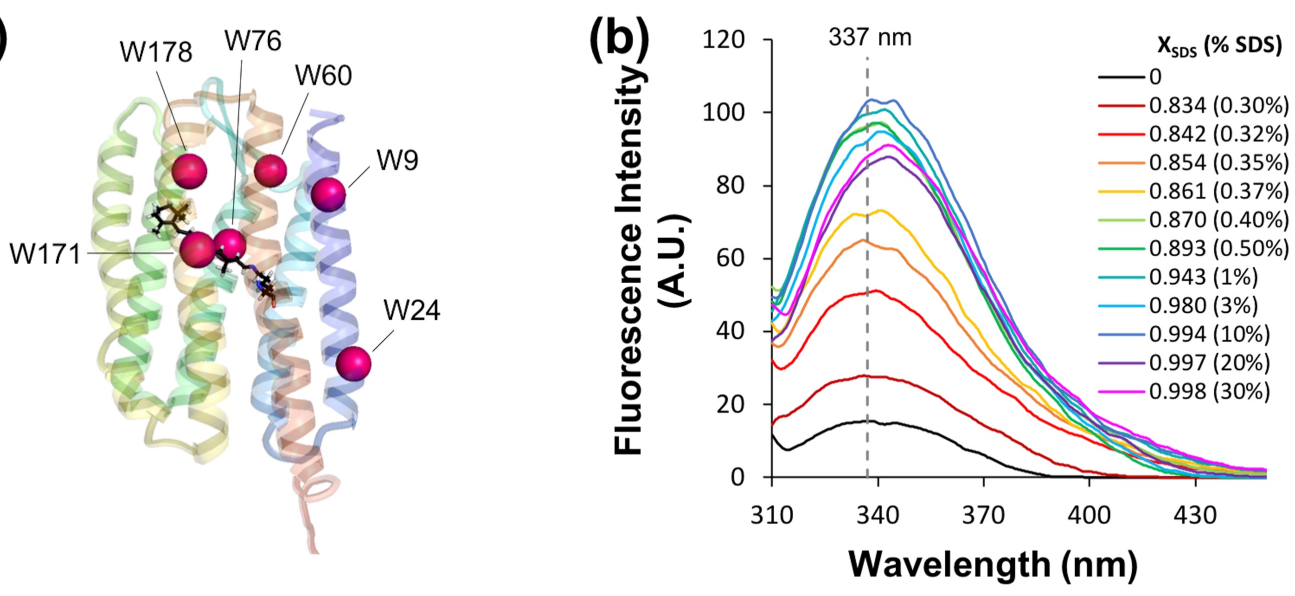




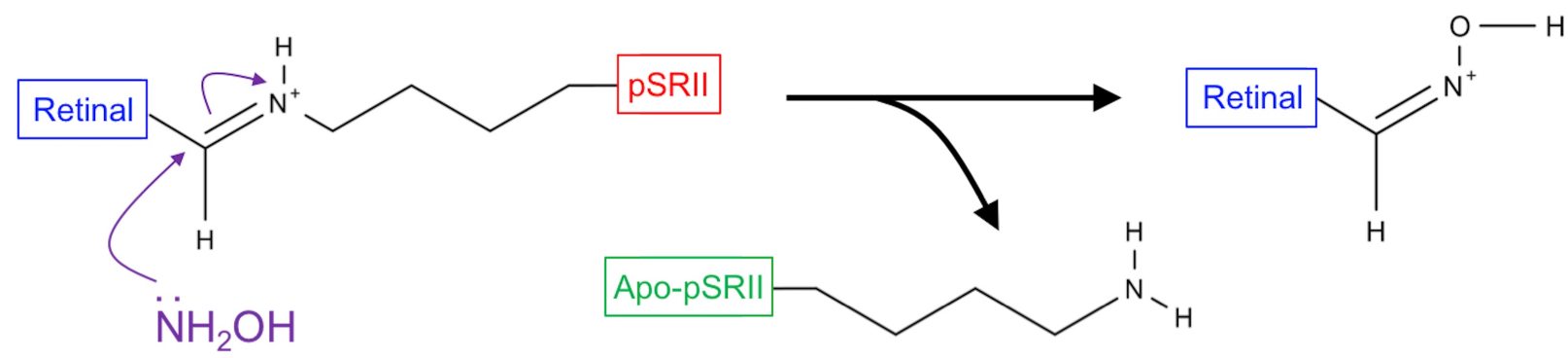

(b)
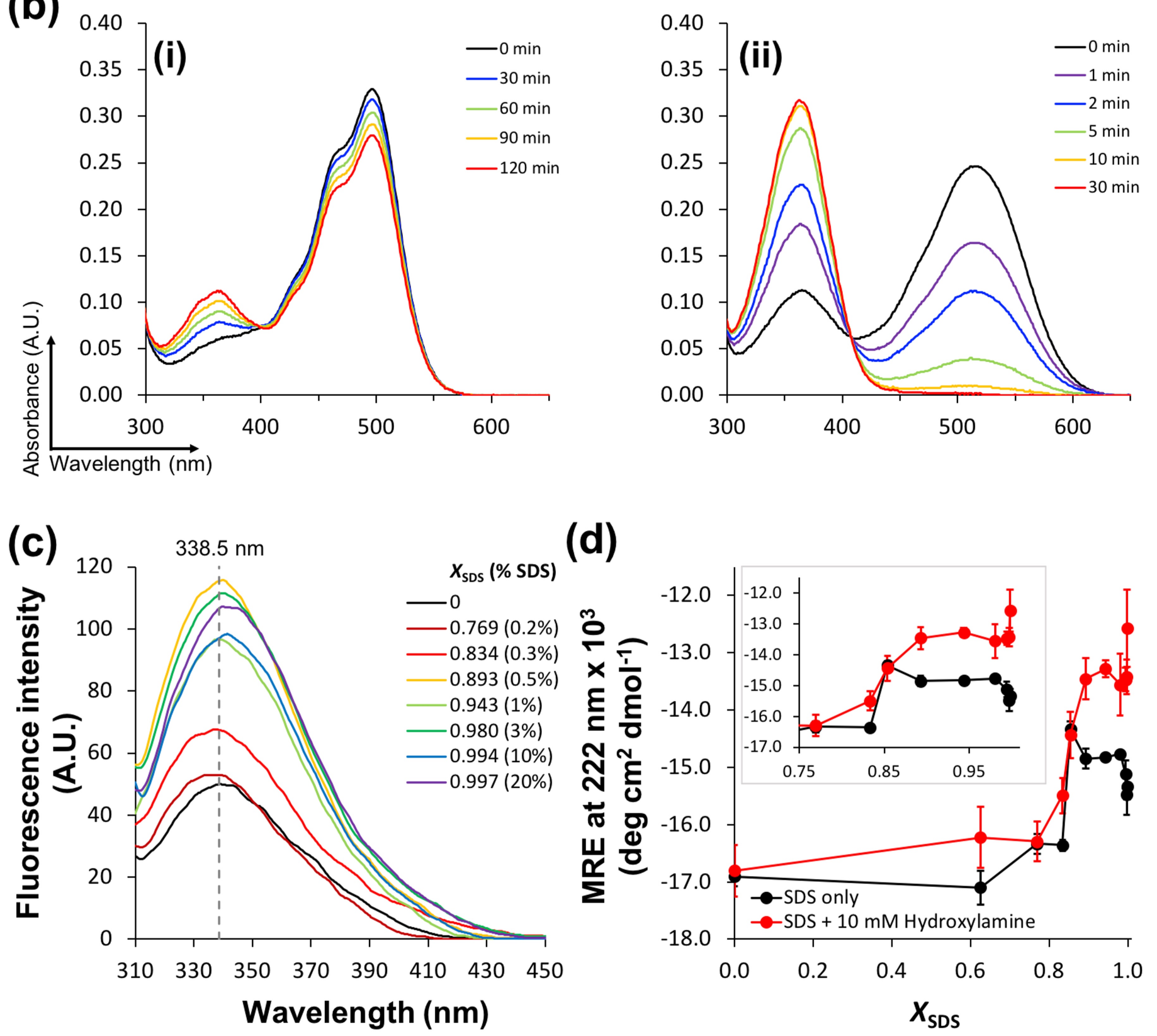
(a)
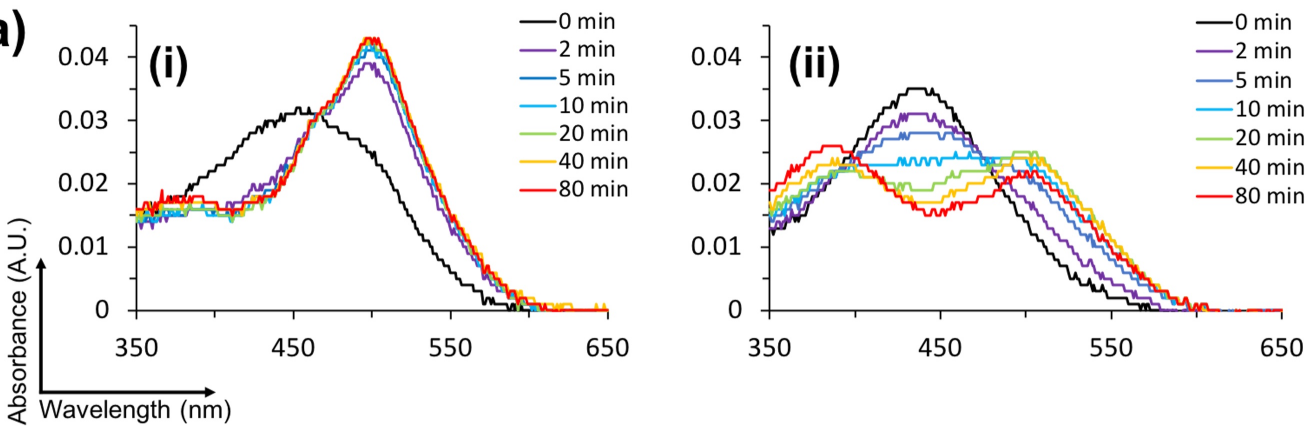

(b)
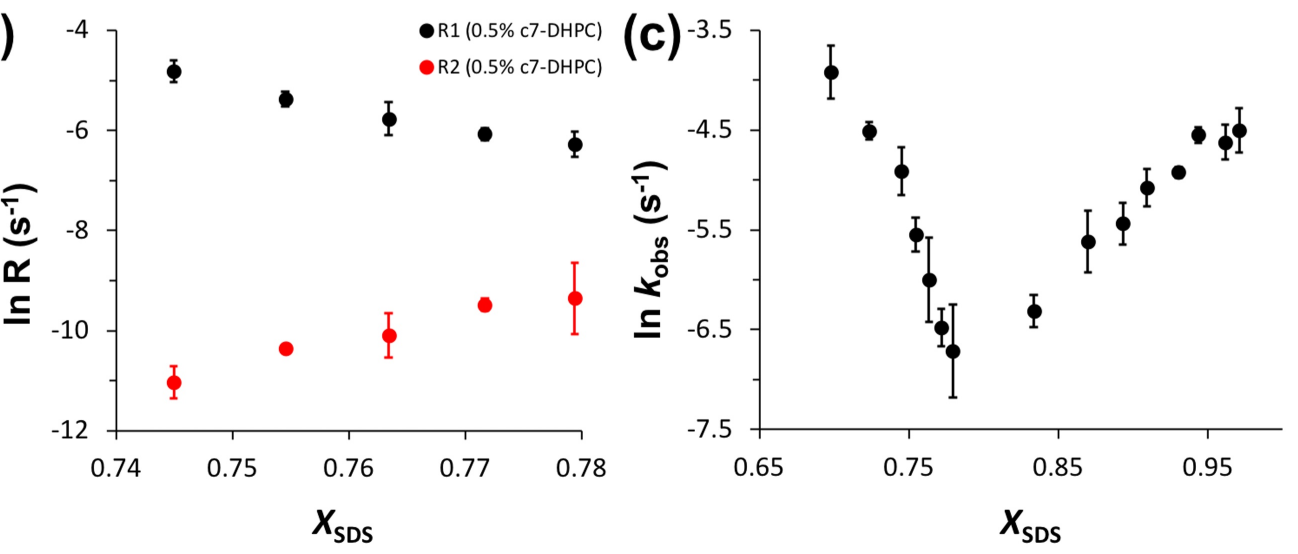

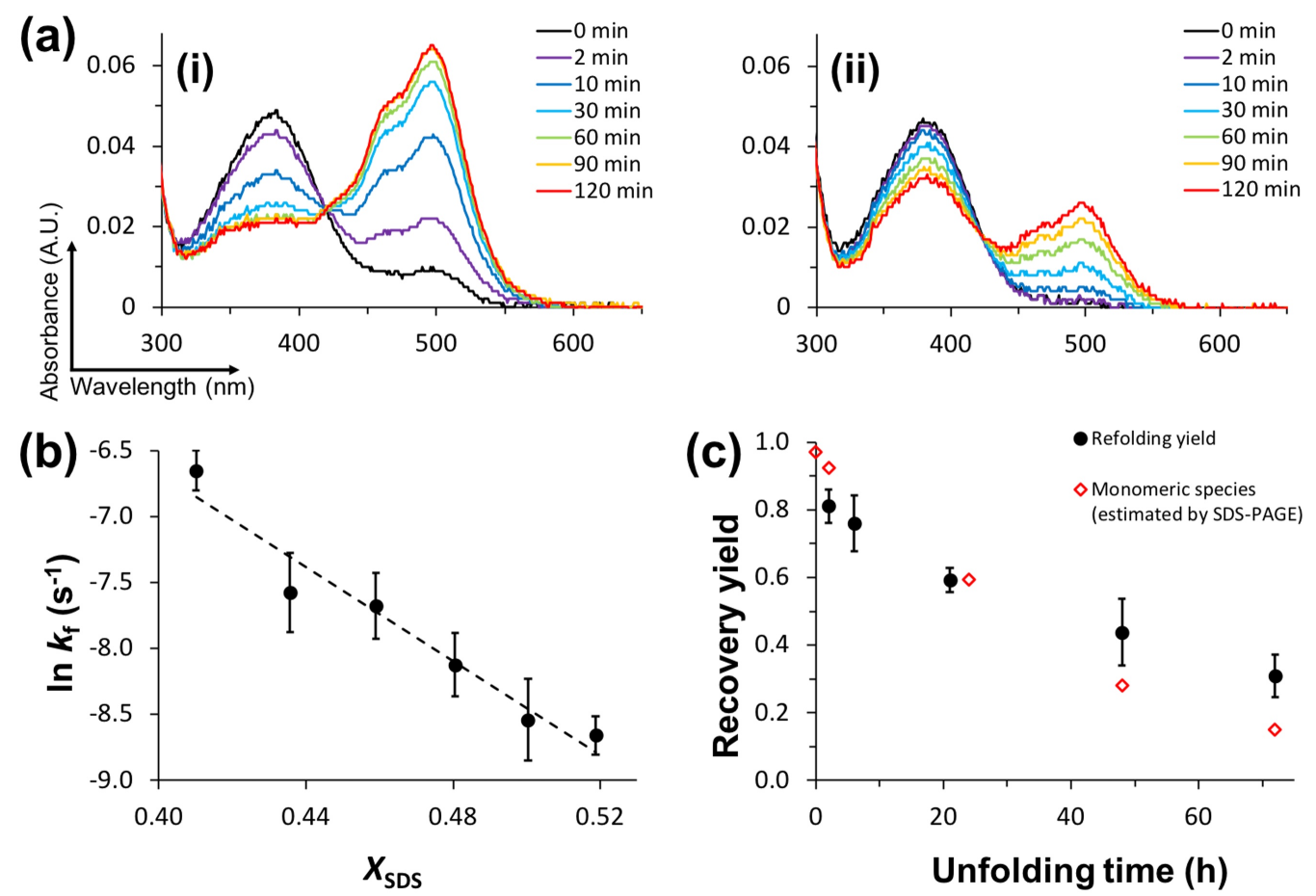


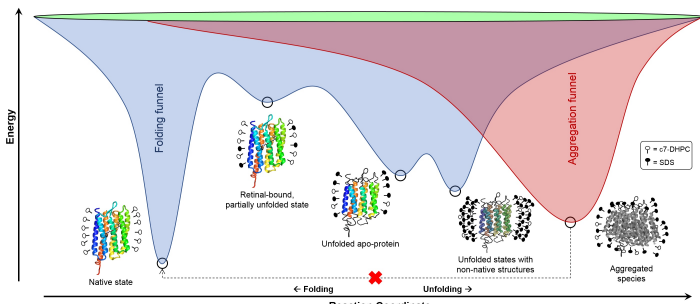




\section{Supplementary Information}

\section{Characterisation of Denatured States and Reversible Unfolding of Sensory Rhodopsin II}

Yi Lei $\operatorname{Tan}^{1}$, James Mitchell ${ }^{2}$, Judith Klein-Seetharaman ${ }^{2}$, Daniel Nietlispach ${ }^{1 *}$

${ }^{1}$ Department of Biochemistry, 80 Tennis Court Road, University of Cambridge, CB2 1GA, United Kingdom

${ }^{2}$ Biomedical Sciences Division, Warwick Medical School, University of Warwick, Coventry, CV4 7AL, United Kingdom

*To whom correspondence should be addressed: Daniel Nietlispach, Department of Biochemistry, 80 Tennis Court Road, University of Cambridge, CB2 1GA, United Kingdom. Phone: +44 1223766023 , Fax: +44 1223 766002, Email: dn206@cam.ac.uk 


\section{SUPPLEMENTARY RESULTS}

\section{Aggregation under different denaturing conditions}

Native loading dye (see Methods) was used so that no additional SDS was introduced from the sample buffer. It is unlikely for SDS present in the gel and running buffer (each containing $0.1 \%$ SDS) to re-dissolve aggregates, as it was subsequently determined that $0.1 \%$ SDS was insufficient to unfold pSRII, and denaturant concentrations in the unfolding buffers were usually much higher than the SDS concentration present in the gel and running buffer.

A detailed analysis of SDS concentration-dependent oligomerisation of pSRII denatured in $0.1-30 \%$ SDS is shown in Fig. S1a. The oligomerised species were hypothesised to represent off-pathway species that cannot be refolded to restore the native chromophore. To optimise the unfolding condition, different additives were explored with the goal of preventing or reducing aggregation, and the results are summarised in Table S2. Protein aggregation was monitored by SDS-PAGE and the extent of protein unfolding was determined by UV/vis spectroscopy (Fig. S2). Additives commonly used for reducing protein aggregation [1] were added to unfolding buffers containing $3 \%$ SDS. Non-detergent sulfobetaines NDSB-201 (3-(1-Pyridinio)-1-propanesulfonate) and NDSB-256 (3-(Benzyldimethylammonio)propanesulfonate), L-Arginine and polyethylene glycol PEG-8000 were explored for stabilising partially unfolded intermediates or sterically interfering with aggregation. LDAO (Lauryldimethylamine-N-oxide), urea and different solvent conditions including salt concentration (0-500 $\mathrm{mM} \mathrm{NaCl})$ and $\mathrm{pH}(\mathrm{pH}$ 1.0-6.0, adjusted using TFA) were also attempted to optimise electrostatic and hydrophobic interactions. Further controls against possible modifications such as trifluorination by TFA were accounted for by unfolding pSRII at $\mathrm{pH} 2.0$, adjusted using phosphoric acid, both in the absence and presence of SDS (Fig. S3). As shown in Fig. S3, the formation of oligomers in SDS at pH 2.0 showed similar dependence on time and SDS concentration as in SDS at pH 6.0 (Fig. 2, main text). Despite these extensive efforts, none of the tested conditions offered decreased oligomerisation without compromising the unfolding rate. For example, $3 \%$ SDS + $0.5 \mathrm{M}$ L-arginine led to minimal amount of aggregation, but the protein did not unfold $\left(\lambda_{\max }=498\right.$ $\mathrm{nm}$; Fig. S2d).

\section{Secondary structure changes in SDS at pH 2.0}

pSRII unfolded in SDS at pH 2.0 also showed little loss in alpha-helical content (Fig. S4). Essentially no difference in MRE at $222 \mathrm{~nm}$ was observed even after exposure of pSRII to acidic $\mathrm{pH}$ for 19 hours, suggesting slow unfolding in the absence of SDS (Fig. S4a(i)). $0.998 X_{\text {SDS }}(30 \%$ SDS in $0.1 \%$ c7-DHPC) at 
$\mathrm{pH} 2.0$ led to fast loss of alpha-helicity within seconds (Fig. S4a(ii)), contrasting against pSRII unfolding in SDS at pH 6.0, where the loss of secondary structure occurs at timescales ranging from seconds to hours, with the rate constant being dependent on $X_{\text {SDS }}$ (Fig. S8). Further loss of alpha-helicity beyond 20 hours in SDS at $\mathrm{pH} 2.0$ is attributed to aggregation, as evidenced SDS-PAGE (Fig. S3) and appearance of local minima at $218 \mathrm{~nm}$ characteristic of increase in beta-sheet content (Fig. S4a).

Thus, similar to the results obtained in SDS at pH 6.0 (Fig. 3a, main text), pSRII unfolded in SDS at pH 2.0 showed little loss in alpha-helical content (Fig. S4), although this occurs in the timescale of seconds rather than timescales ranging from seconds to hours as in SDS at pH 6.0.

\section{Changes in retinal binding pocket in SDS at $\mathrm{pH} 2.0$}

Acid denaturation of pSRII at pH 2.0 (without SDS) led to very slow protein unfolding and Schiff base hydrolysis, with the reaction taking between 5 and 24 hours to reach completion (Fig. S6a(i)). pSRII in $\geq 0.625 X_{\text {SDS }}(0.1 \%$ SDS in $0.1 \% \mathrm{c} 7-\mathrm{DHPC})$ at $\mathrm{pH} 2.0$ led to the formation of $\mathrm{SR}_{440}$ within the dead-time of 25 seconds, followed by very slow release of free retinal over a period of more than 6 days (Fig. S6a(ii)). This indicates that in an acidic environment, the retinal binding pocket is structurally disrupted within seconds after initial exposure to SDS but Schiff base hydrolysis is significantly slowed down. These observations are consistent with previous reports of bacteriorhodopsin in DMPC/CHAPSO micelles being unfolded at $\mathrm{pH} 1.2$ in the presence of $6 \mathrm{mM}$ SDS [2]. pSRII unfolded in SDS at pH 2.0 also showed less aggregation than at pH 6.0 (Figs. 2, S1 \& S3). Altogether, these observations suggest that the attached retinal chromophore might be exerting a stabilising effect against protein aggregation.

Hence, in comparison with pH 6.0 (Fig. 4, main text), acid denaturation of pSRII at pH 2.0 (without SDS) led to very slow protein unfolding and Schiff base hydrolysis. Addition of SDS led to disruption of the retinal binding pocket within seconds after initial exposure to SDS but Schiff base hydrolysis is significantly slowed down.

\section{Tertiary Structure Changes in SDS at pH 2.0}

Tryptophan fluorescence of pSRII in the absence of SDS at pH 2.0 also has $\lambda_{\max }$ of $337 \mathrm{~nm}$, indicative of a similar environment surrounding the tryptophan residues at both $\mathrm{pHs}$. The presence of 0.769 $X_{\mathrm{SDS}}$ led to burial of tryptophan residues into a more hydrophobic environment, as evidenced by the spectral blue-shift to $332 \mathrm{~nm}$. Further addition of SDS led to a spectral red-shift to $340 \mathrm{~nm}$ at 
$0.998 X_{\text {SDS }}(30 \%$ SDS in $0.1 \%$ c7-DHPC) (Fig. S7a(i)), similar to pSRII unfolded in SDS at pH 6.0, as the tryptophan residues become more solvent-exposed. Akin to the results at pH 6.0 (Fig. 5, main text), pSRII in SDS at pH 2.0 also showed higher fluorescence intensities and solvent exposure with increasing $X_{\text {SDS. }}$. Fluorescence intensities remained constant over 30 minutes (Fig. S7a(ii)), consistent with the lack of tertiary structure changes over the same timescale observed by UV/vis spectroscopy. On the other hand, fluorescence intensities increased slowly in $0 X_{S D S}$ at $\mathrm{pH} 2.0$, consistent with the very slow protein unfolding and Schiff base hydrolysis in $0 X_{\text {SDS }}$ observed by UV/vis spectroscopy (Fig. S6a).

\section{Solvent Accessibility of the Binding Pocket in SDS at pH 2.0}

$10 \mathrm{mM}$ hydroxylamine at $\mathrm{pH} 2.0$ led to near-complete formation of retinal oxime after 5 hours (Fig. $\mathrm{S6b}(\mathrm{i})$ ), indicating that the acidic $\mathrm{pH}$ enabled greater solvent accessibility of the retinal binding pocket than at $\mathrm{pH}$ 6.0. The loss of native chromophore in the presence of hydroxylamine at $\mathrm{pH} 2.0$ was faster than in the absence of hydroxylamine (Fig. S6c). Addition of SDS also led to fast denaturation such that only the $\mathrm{SR}_{440}$ intermediate was observed within the experimental dead-time (Fig. S6b(ii)). However, the reaction did not reach completion even after 90 hours (cf. complete reaction within 30 minutes at $\mathrm{pH} 6.0$ shown in Fig. $6 \mathrm{~b}(\mathrm{ii})$ ), as both retinal oxime and retinal PSB (440 $\mathrm{nm}$ ) were visible, manifesting as a very broad absorbance peak at $~ 410 \mathrm{~nm}$. This suggests that the reaction of hydroxylamine with retinal Schiff base is significantly slowed down at $\mathrm{pH}$ 2.0. The presence of hydroxylamine at $\mathrm{pH} 2.0$ led to smaller loss of secondary structure within 30 minutes (Fig. S4b), possibly due to incomplete Schiff base cleavage by hydroxylamine at $\mathrm{pH} 2.0$ within this time frame, hence resulting in partial stabilisation of some elements of pSRII secondary structure as hypothesised above for pSRII molecules which still harbour PSB. The same unfolding transition point was observed, regardless of the absence or presence of hydroxylamine (Fig. S4b). Fluorescence intensities increased very slowly over time, with slower rates being observed at higher $X_{\text {SDS }}$ (Fig. S7b(ii)), suggesting that SDS might create a 'shielding' effect at $\mathrm{pH} 2.0$, either by occluding access of hydroxylamine to the retinal binding pocket or by causing a decrease in the reactivity of hydroxylamine.

In summary, the reaction of hydroxylamine with retinal Schiff base at both pH 6.0 and pH 2.0 were dependent on accessibility to the binding pocket, which increased in the presence of SDS. However, this reaction was significantly slowed down at $\mathrm{pH}$ 2.0, leading to incomplete Schiff base cleavage and smaller secondary and tertiary structure changes. 


\section{Non-linearities in the Folding Arm of the Chevron Plot}

Non-linearities in chevron plots could be attributed to multiple micelle occupancy with a protein that has tendency to oligomerise, transient aggregates or kinetic intermediates [3]. To determine whether non-linearity in the folding arm is due to any of these factors, unfolding and refolding time-courses were recorded in increased detergent-to-protein ratio [4], obtained by increasing detergent concentration and/or decreasing protein concentration. The unfolding (Fig. S13a) and refolding (Fig. S13b) rate constants obtained were superimposable with those in Fig. 7c. SDS-PAGE of samples taken at various time points up to 2 hours of the refolding reaction in different $X_{\text {SDS }}$ also showed no increase in the amount of stably-formed oligomers compared to folded pSRII (Fig. S13c). These results indicate that there is no evidence for aggregation or multiple micelle occupancy being the origin of non-linearity in the chevron plot, suggesting a more complex refolding mechanism for pSRII. 


\section{Supplementary Discussion}

\section{Comparison across different biophysical techniques}

To compare structural insights across different conditions and biophysical techniques, changes over time of MRE at $222 \mathrm{~nm}$, retinal UV/vis absorbance, and tryptophan fluorescence emission at $335 \mathrm{~nm}$ were recorded for pSRII unfolded in different $X_{\text {SDS }}$ in $0.1 \%$ c7-DHPC at pH 6.0, both in the absence and presence of $10 \mathrm{mM}$ hydroxylamine. The time courses were fitted to the appropriate models using non-linear least squares fitting (see Methods).

These rate constants differ slightly from those reported for pSRII unfolding in SDS + 0.5\% c7-DHPC (see Table 1, main text) due to possible complications with multiple micelle occupancy under low c7-DHPC concentrations. Nevertheless, good consistency was obtained across the three techniques, indicating that rate constants extracted by UV/vis spectroscopy for pSRII unfolding in SDS $+0.5 \%$ c7-DHPC would have been comparable to those determined by other techniques. Moreover, comparable unfolding transitions were obtained across the three biophysical techniques (cf. Figs. 3b, $4 \mathrm{~b}$ and 59$)$.

Secondary structure changes occur as a one-step process, as evidenced by good monoexponential fits of changes in MRE at $222 \mathrm{~nm}$ (Fig. S10a). Similar rate constants were obtained regardless of the absence or presence of hydroxylamine (Fig. S8). Tertiary structure changes, monitored by UV/vis spectroscopy and tryptophan fluorescence time-courses, occur in two steps: protein unfolding to form $\mathrm{SR}_{440}$ and Schiff base hydrolysis to yield $\mathrm{SO}_{390}$, respectively. The rate constants extracted from tryptophan fluorescence time-courses were similar to those from UV/vis time-courses for most $X_{\mathrm{SDS}}$, hence confirming that the increase in fluorescence intensity during unfolding was primarily due to un-quenching of tryptophan fluorescence while retinal was being removed from its binding pocket (Fig. S8a). At $0.893 X_{\text {SDS }}\left(0.5 \%\right.$ SDS) and $0.943 X_{\text {SDS }}(1 \%$ SDS), the unfolding rate constant measured by tryptophan fluorescence was smaller than that measured by UV/vis spectroscopy, suggesting that fluorescence remains partially quenched for one or more tryptophan residues near the partially loosened retinal binding pocket. Schiff base hydrolysis was fast in the presence of hydroxylamine and SDS at $\mathrm{pH}$ 6.0, such that retinal oxime formed rapidly without accumulation of the $\mathrm{SR}_{440}$ intermediate, and only one rate constant was extracted across all biophysical techniques (Fig. S8b).

In summary, exposure of pSRII to SDS led to $X_{\text {SDS }}$-dependent protein unfolding followed by $X_{\mathrm{SDS}}$-independent retinal Schiff base hydrolysis. The rate limiting step depends on the relative rates of these two processes, and governs the rate constant(s) that can be extracted from the time-courses. 


\section{SDS-denatured states of pSRII at pH 2.0}

Acid denaturation of pSRII in $0 X_{\text {SDS }}$ led to small structural changes, as evidenced by the small increase in solvent accessibilities of retinal and tryptophan residues and also the slow bleaching of pSRII in the presence of hydroxylamine. pSRII in SDS at pH 2.0 showed very fast loosening of the retinal binding pocket within seconds, but extremely slow retinal Schiff base hydrolysis on the timescale of days, rather than minutes to hours as in $\mathrm{pH} 6.0$. Increase in solvent accessibility of tryptophan residues and bound retinal were also slowed down to timescales matching that of retinal Schiff base hydrolysis, whereas loss in alpha-helicity was accelerated to within minutes. In comparison to $\mathrm{pH}$ 6.0, a greater extent of alpha-helical content was preserved at $\mathrm{pH}$ 2.0. A detailed comparison between the apo-protein states $\left(\mathrm{SO}_{390}\right)$ in SDS at $\mathrm{pH} 2.0$ and $\mathrm{pH} 6.0$, however, would be complicated by changes in Schiff base reactivity at acidic $\mathrm{pH}$ and aggregation over prolonged time.

Although the presence of retinal in retinal-binding proteins enables convenient monitoring of unfolding and refolding by spectroscopic techniques, this also leads to complications with further studies of $\mathrm{SR}_{440}$ (denatured pSRII with covalently-bound retinal) due to fast protein conformational changes and very slow retinal Schiff base hydrolysis occurring simultaneously. SR 440 $_{\text {in }}$ SDS at pH 2.0 has caught our attention because its decay due to retinal Schiff base hydrolysis was significantly slowed down. Further structural studies are required to understand to what extent $\mathrm{SR}_{440}$ at $\mathrm{pH} 6.0$ and at $\mathrm{pH} 2.0$ are structurally similar to each other, and whether $\mathrm{SR}_{440}$ at $\mathrm{pH} 2.0$ could be used as a substitute for further structural characterisation.

We also note that pSRII in SDS at $\mathrm{pH} 2.0$, which is predominantly $\mathrm{SR}_{440}$, aggregated slower than pSRII in SDS at $\mathrm{pH}$ 6.0, which is predominantly $\mathrm{SO}_{390}$. This suggests possible roles of the bound retinal in preventing exposure of aggregation-prone surfaces and in the folding of pSRII. More detailed structural studies will hopefully clarify whether there are protein conformational changes during retinal Schiff base formation in pSRII.

\section{Assessment of pSRII aggregation by SDS-PAGE}

In our study of pSRII aggregation by SDS-PAGE (Fig. S1a), a broadening of SDS-PAGE bands was observed for protein samples with high SDS concentrations. The broadening of bands for samples with high SDS concentrations indicates that these bands fail to be focused into one tight band in the 
stacking gel, consistent with previously published observation of gel band broadening in proportion to SDS load [5].

The irregular migration could be due to the much higher SDS-to-pSRII ratio, resulting in changes in charge and electrophoretic mobility. The SDS concentrations used in SDS-PAGE ranged from $1 \mathrm{mg} / \mathrm{ml}$ $(0.1 \% \mathrm{SDS})$ to $300 \mathrm{mg} / \mathrm{ml}$ (30\% SDS). These concentrations span from sub-CMC concentrations to concentrations which allow the formation of spherical micelles $(0.2-3 \%$ SDS) and/or cylindrical micelles (> 3\% SDS). Micellar SDS has also been proposed to migrate differently to monomeric SDS [5], hence contributing to band broadening. Moreover, the formation of cylindrical micelles in samples with high SDS concentrations could also contribute towards anomalous migration due to the much bigger hydrodynamic radius. Anomalous electrophoretic behaviour due to big hydrodynamic radii was previously reported for the SDS/histone H5 complex [6]. 


\section{SUPPLEMENTARY FIGURES}

(a) $\quad$ pSRIII $0.2 \quad 0.5 \quad 2 \quad 7 \quad 7 \quad 20$ [SDS] (\%) M $|0.1| 0.3|1| 3|10| 30$

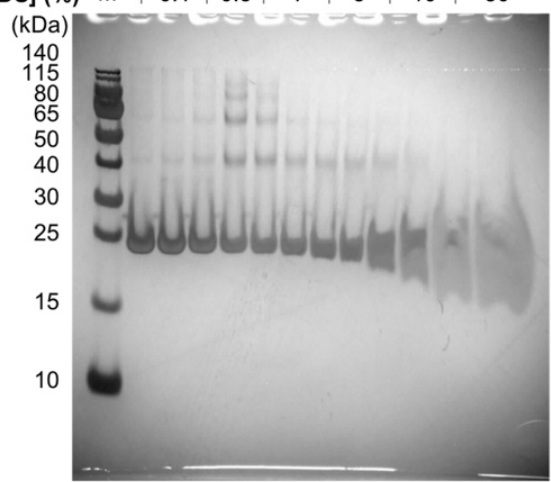

(c)

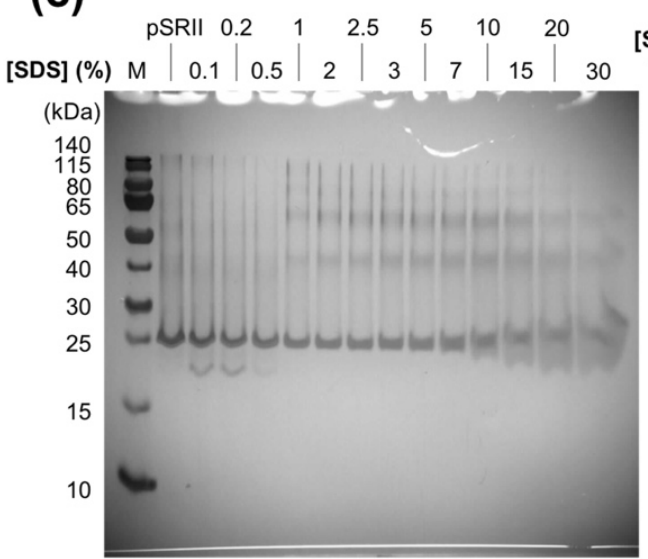

(e)

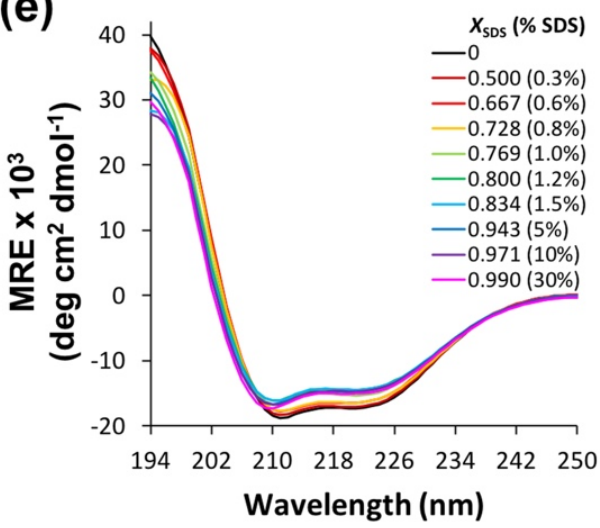

(b)

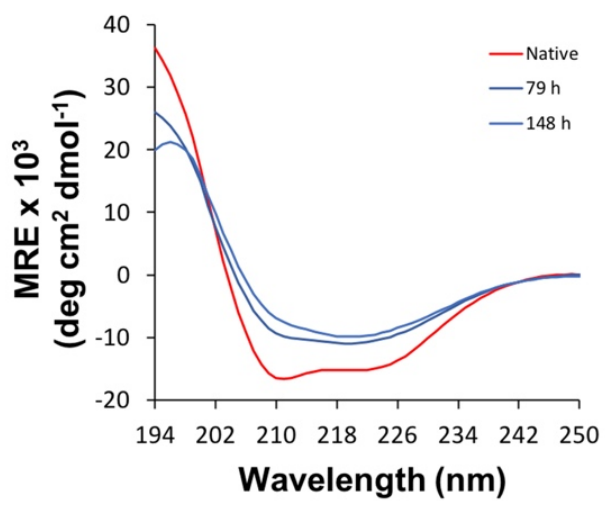

(d) [SDS] (\%)
Time (h) $\quad M \frac{0 \% \text { SDS }}{0222472} \frac{2.5 \% \text { SDS }}{02244872} \frac{15 \% \text { SDS }}{02244872}$

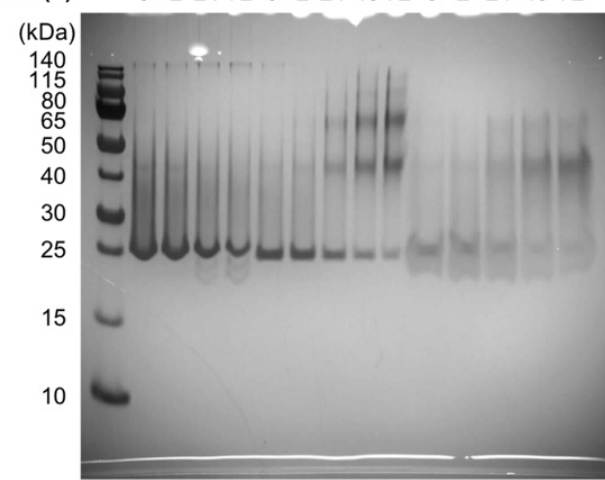

(f)

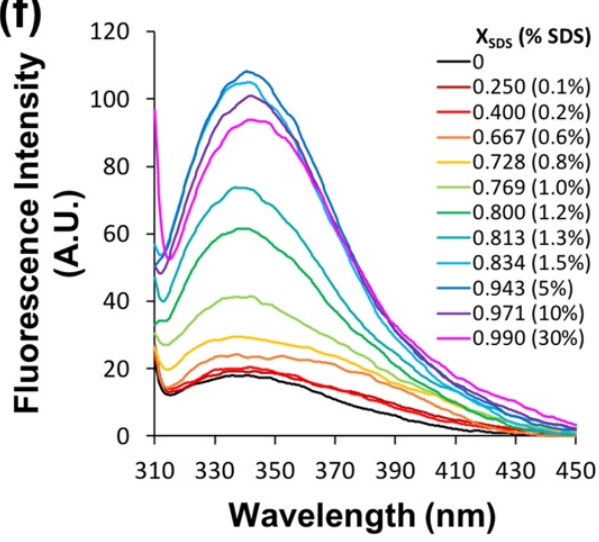

Figure S1. (a) Silver-stained 12\% SDS-PAGE gel of pSRII unfolded in different concentrations of SDS + $0.1 \% \mathrm{c7}$-DHPC at pH 6.0 for $14 \mathrm{~h}$ at $25^{\circ} \mathrm{C}$. Lane 1, molecular weight marker; lane 2, pSRII; lanes 3-13, pSRII in $0.1-30 \%$ SDS. (b) CD spectra of $7 \mu \mathrm{M}(0.2 \mathrm{mg} / \mathrm{ml})$ native pSRII and pSRII unfolded in 0.998 $X_{\text {SDS }}(30 \%$ SDS in $0.1 \%$ c7-DHPC), pH 6.0 for up to $148 \mathrm{~h}$, showing loss of alpha-helical structure and gain of beta-sheet structure over time. (c) Silver-stained 12\% SDS-PAGE gel of pSRIl unfolded in different concentrations of SDS $+0.5 \% \mathrm{c} 7$-DHPC at pH 6.0 for $14 \mathrm{~h}$ at $25^{\circ} \mathrm{C}$. Lane 1 , molecular weight marker; lane 2, pSRII; lanes 3-13, pSRII in 0.1-30\% SDS. (d) Silver-stained 12\% SDS-PAGE gel showing increased aggregation over prolonged unfolding time of pSRII in $0 \%$ (lanes 2-5), 2.5\% (lanes 6-10) and $15 \%$ SDS (lanes 11-15) + 0.5\% c7-DHPC at pH 6.0 for $0-72 \mathrm{~h}$ at $25^{\circ} \mathrm{C}$. (e-f) CD spectra (e) and tryptophan fluorescence emission spectra (f) for $7 \mu \mathrm{M}(0.2 \mathrm{mg} / \mathrm{ml})$ pSRII in $0-0.990 X_{\text {SDS }}(0-30 \%$ SDS in $0.5 \%$ c7-DHPC). 


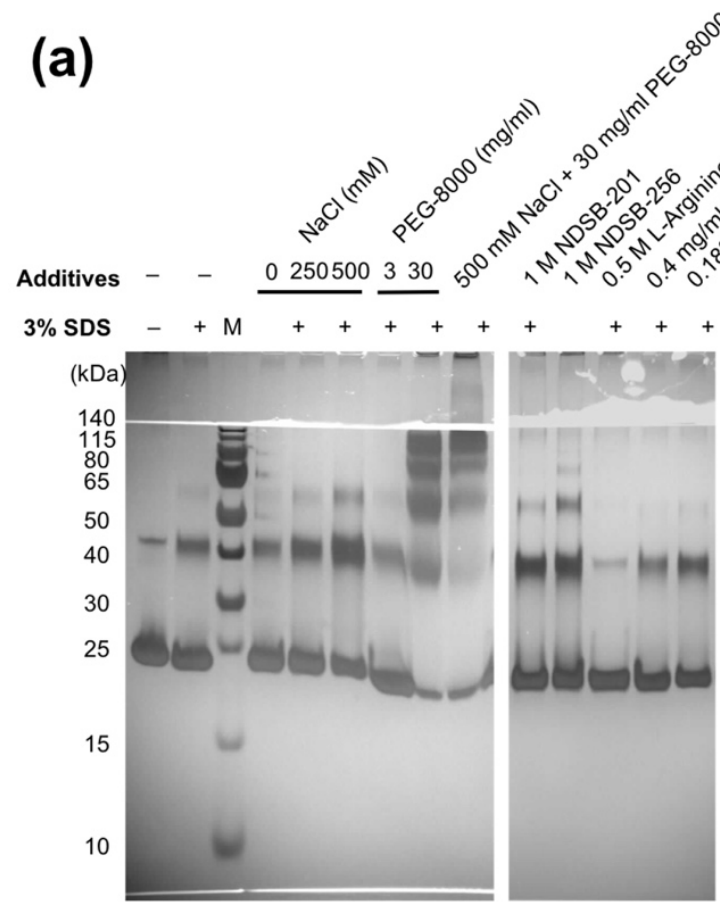

(b)

(c)

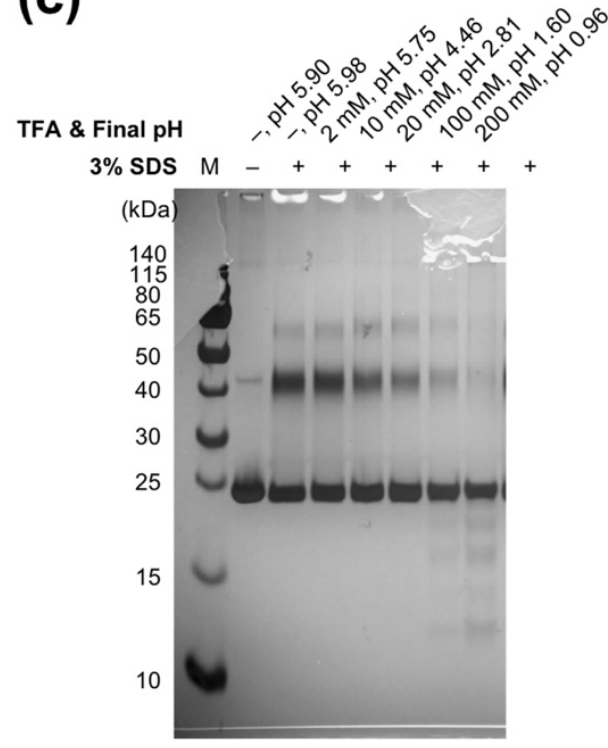

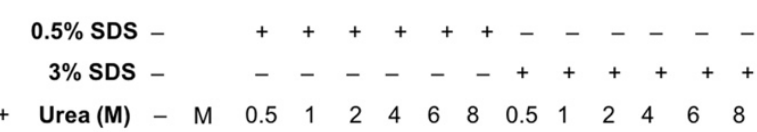

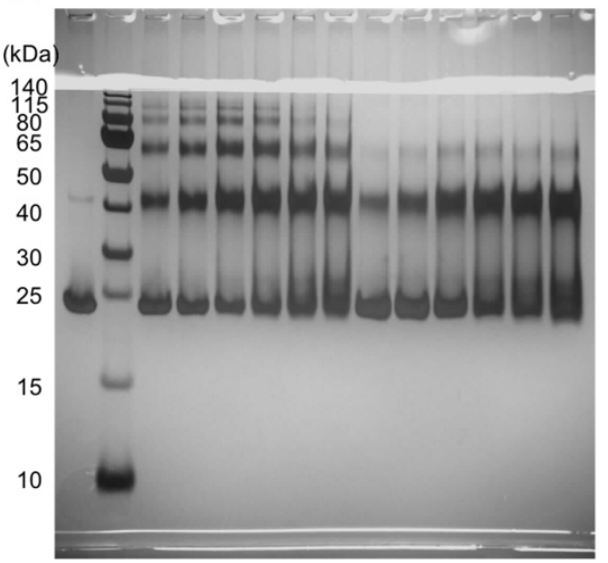

(d)

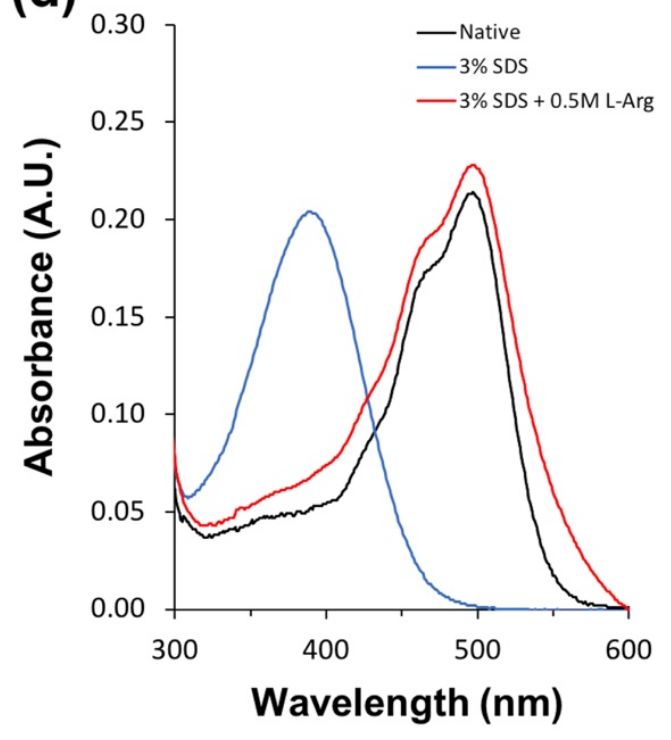

Figure S2. Denaturation of pSRII in SDS leads to some extent of aggregation over time. (a) Silver-stained $12 \%$ SDS-PAGE gel of pSRII unfolded for 16 hours in SDS in the presence of different additives, in attempt to decrease oligomerisation. (b) SDS-PAGE of pSRII unfolded in SDS + Urea after 14.5 hours. (c) SDS-PAGE of pSRII in 3\% SDS + varied concentrations of TFA after 62.5 hours shows $\mathrm{pH}$-dependent unfolding, aggregation and degradation. (d) UV/vis spectra of the retinal chromophore of pSRII in the native condition (black trace), 3\% SDS (blue trace) and 3\% SDS + $0.5 \mathrm{M}$ L-arginine (red trace) show that L-arginine has prevented the protein from unfolding. 
(a)

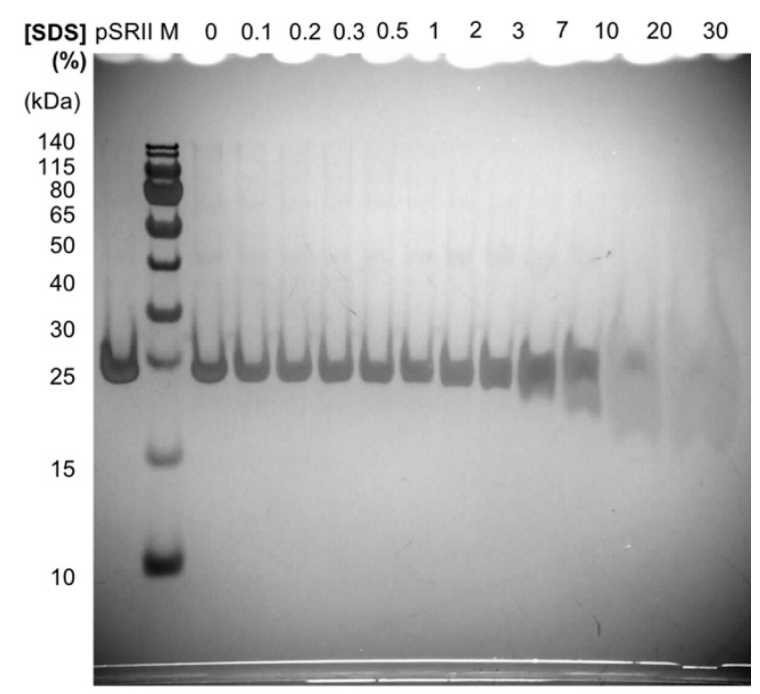

(b)

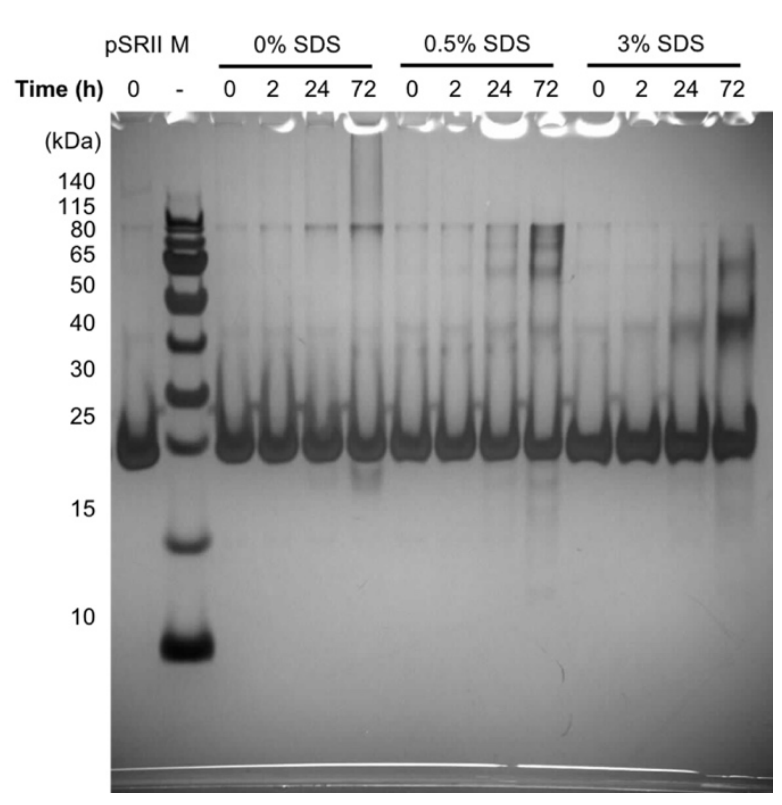

Figure S3. (a) SDS-PAGE of pSRII unfolded in different concentrations of SDS + 0.1\% c7-DHPC at pH 2.0 for $14 \mathrm{~h}$ at $25^{\circ} \mathrm{C}$. Lane $1, \mathrm{pSRII}$ at $\mathrm{pH} 6.0$; lane 2, molecular weight marker; lanes 3-14, pSRII in $0-$ $30 \%$ SDS at $\mathrm{pH}$ 2.0. (b) SDS-PAGE showing time-dependent aggregation of pSRII unfolded in SDS at $\mathrm{pH}$ 2.0. Lane 1, pSRII at pH 6.0; lane 2, molecular weight marker; lanes 3-14, pSRII unfolded for 0 min, $2 \mathrm{~h}, 24 \mathrm{~h}$ and $72 \mathrm{~h}$ in $0 \%$ (lanes $2-5$ ), 0.5\% (lanes 6-9) and 3\% SDS (lanes 10-13), pH 2.0. 

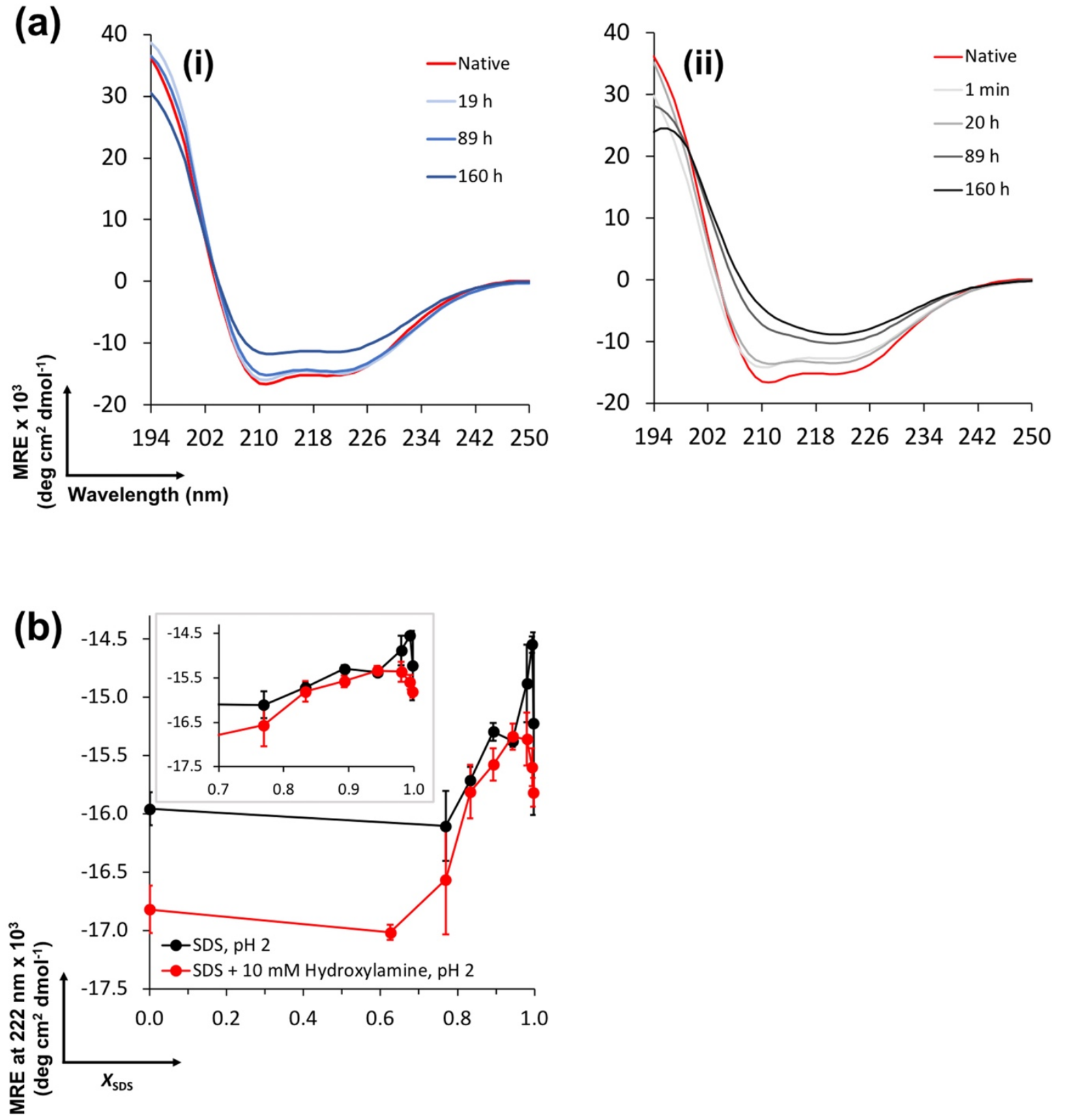

Figure S4. Circular dichroism of pSRII in SDS + 0.1\% c7-DHPC at pH 2.0. (a) CD spectra of $7 \mu \mathrm{M}(0.2$ $\mathrm{mg} / \mathrm{ml}$ ) native $\mathrm{pSRII}$ and pSRII unfolded in (i) $0 X_{\text {SDS }}$ and (ii) $0.998 X_{\text {SDS }}(30 \% \mathrm{SDS}$ ) at $\mathrm{pH} 2.0$ for up to $160 \mathrm{~h}$. (b) MRE at $222 \mathrm{~nm}$ for pSRII in 0-0.998 $X_{\text {SDS }}(0-30 \%$ SDS) at pH 2.0 in the absence (black trace) and presence (red trace) of $10 \mathrm{mM}$ hydroxylamine. Inset shows expanded view of the $0.7-1.0 X_{S D S}$ region. Error bars represent the standard deviation of 3 independent measurements. 
$H(r)$

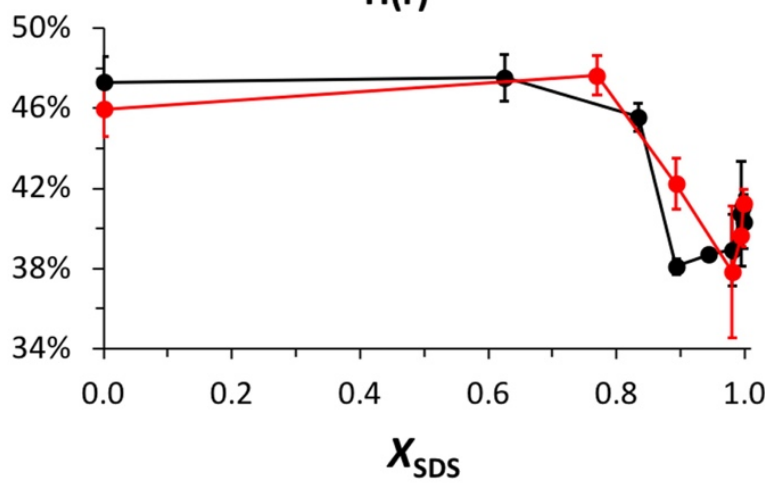

$S(r)$

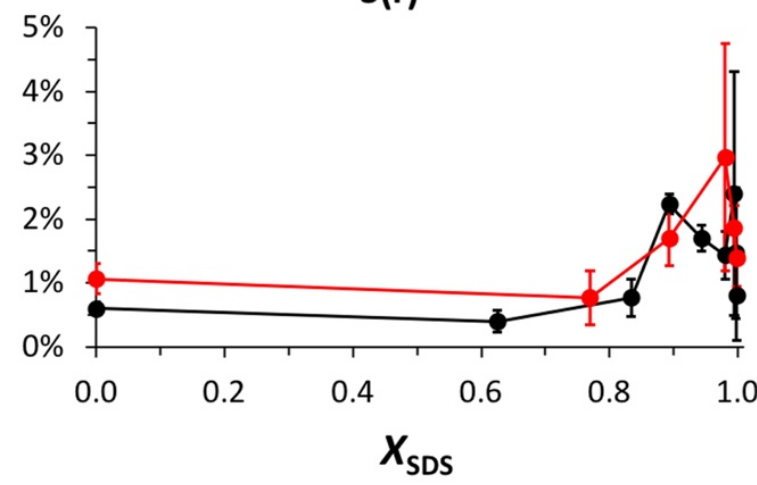

Turn

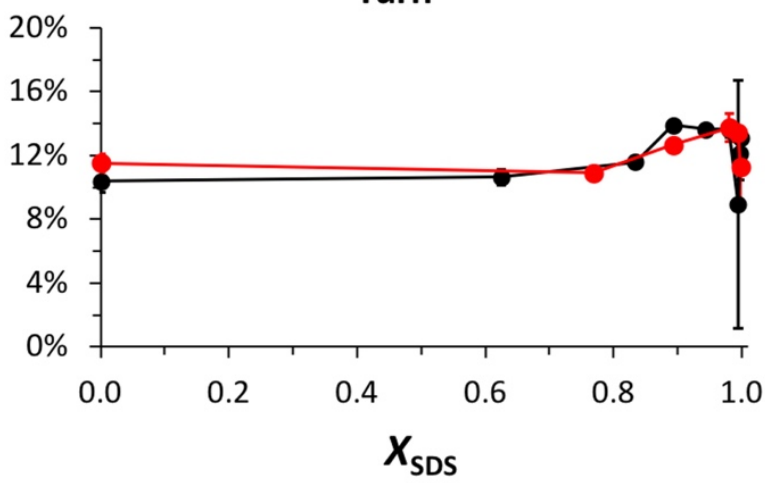

$H(d)$

$\rightarrow-\mathrm{pH} 6.0$

- pH 2.0

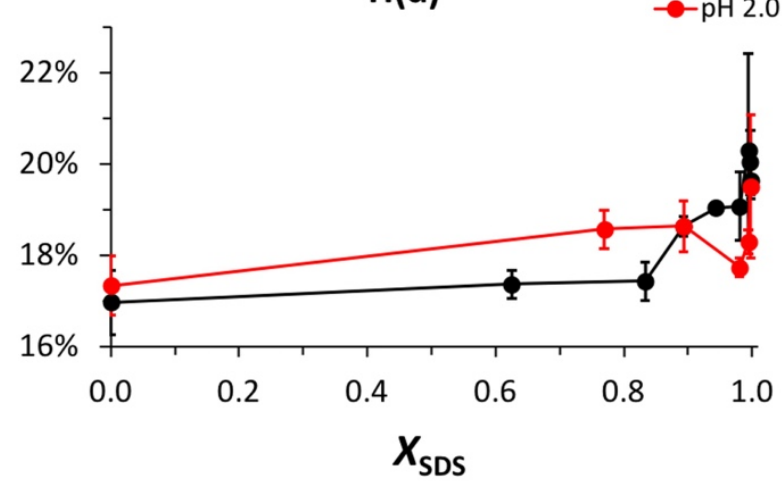

$S(d)$

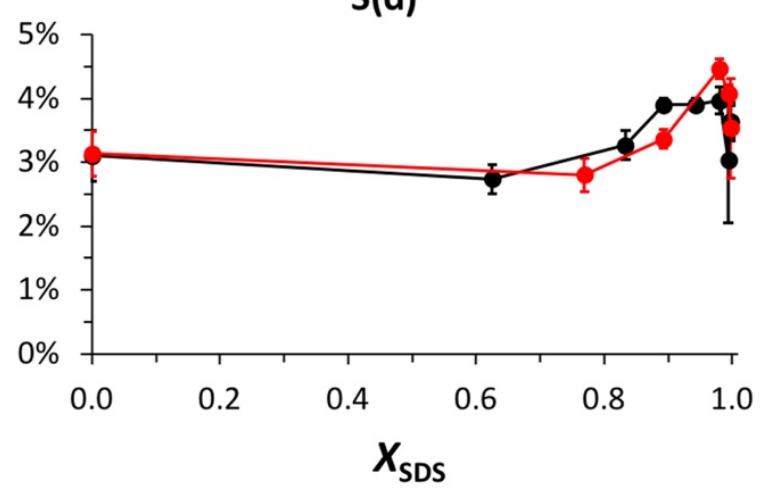

Unrd

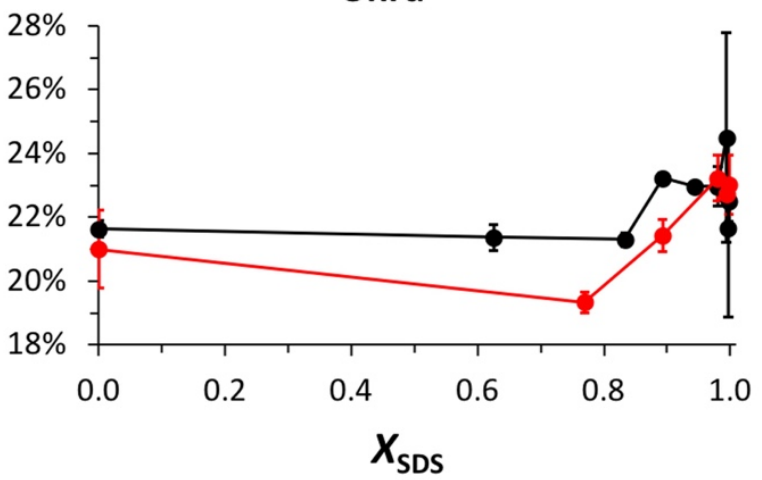

Figure S5. Changes in secondary structure composition of pSRII in SDS at pH 6.0 (black traces) and at pH 2.0 (red traces). Circular dichroism spectra were deconvoluted using CDPro with CONTINLL algorithm and basis set no. 10 (contains soluble and membrane proteins). $H(r)$, regular helix; $H(d)$, distorted helix; $S(r)$, regular strand; $S(d)$, distorted strand; $T$, turns; $U$, unordered. Errors are reported as standard deviations of 3 independent measurements. 

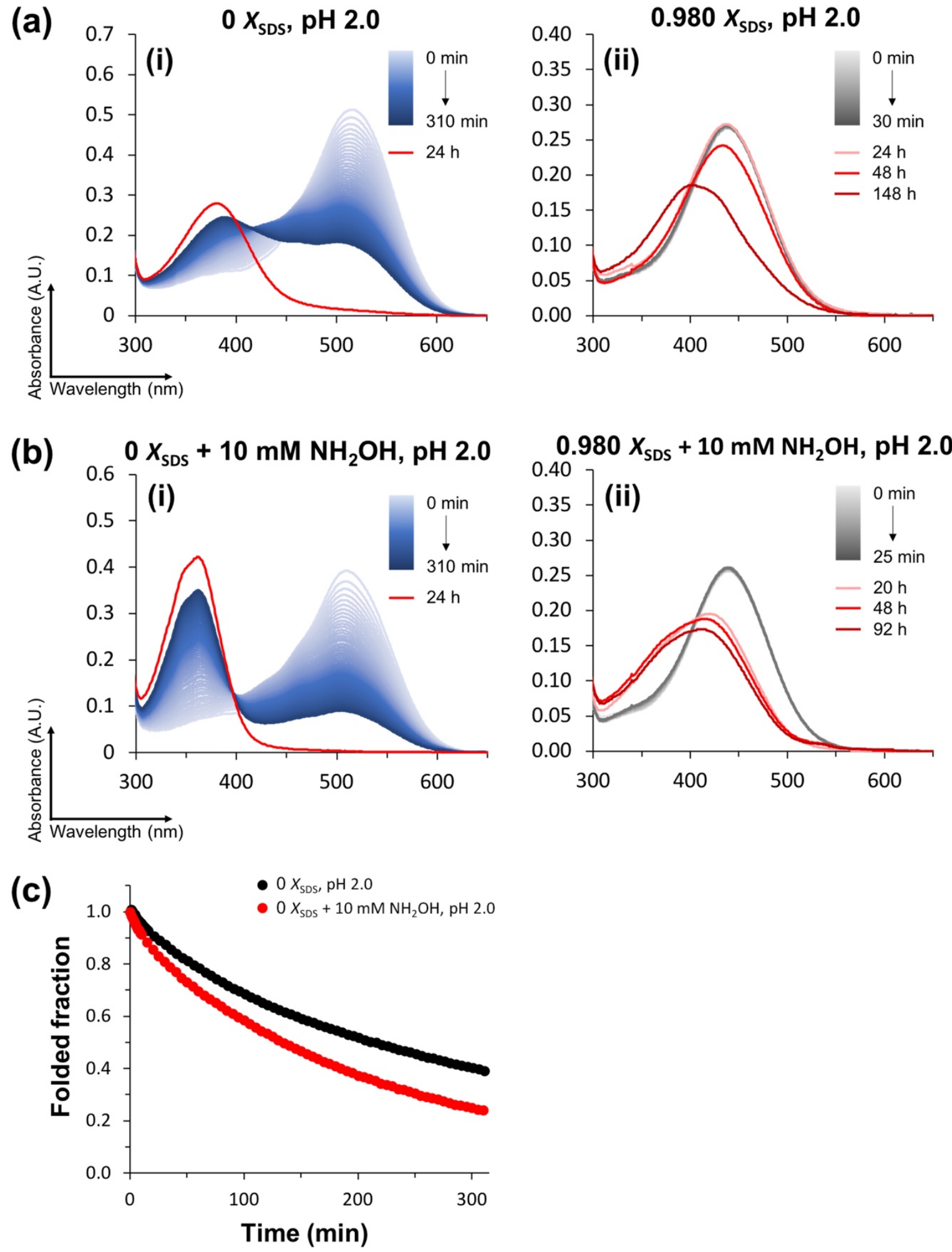

Figure S6. Time-resolved UV/vis spectra of pSRII unfolded at pH 2.0 in $0 X_{\text {SDS }}(a(i), b(i))$ or $0.980 X_{\text {SDS }}$ (3\% SDS) (a(ii), b(ii)), in the absence (a) or presence (b) of $10 \mathrm{mM}$ hydroxylamine. Spectra in panels $\mathbf{a}(\mathbf{i})$ and $\mathbf{b}$ (i) were recorded at 5 -minute intervals and spectra in panels a(ii) and $\mathbf{b}$ (ii) were recorded at 1-minute intervals. Spectra recorded after prolonged periods of unfolding ( $\geq 20 \mathrm{~h}$ ) are plotted in different shades of red. (c) Time-course for the decrease in folded fraction for pSRII in $0 X_{\text {SDS }}$ in the absence or presence of $10 \mathrm{mM}$ hydroxylamine monitored by decay in absorbance at $498 \mathrm{~nm}$. 

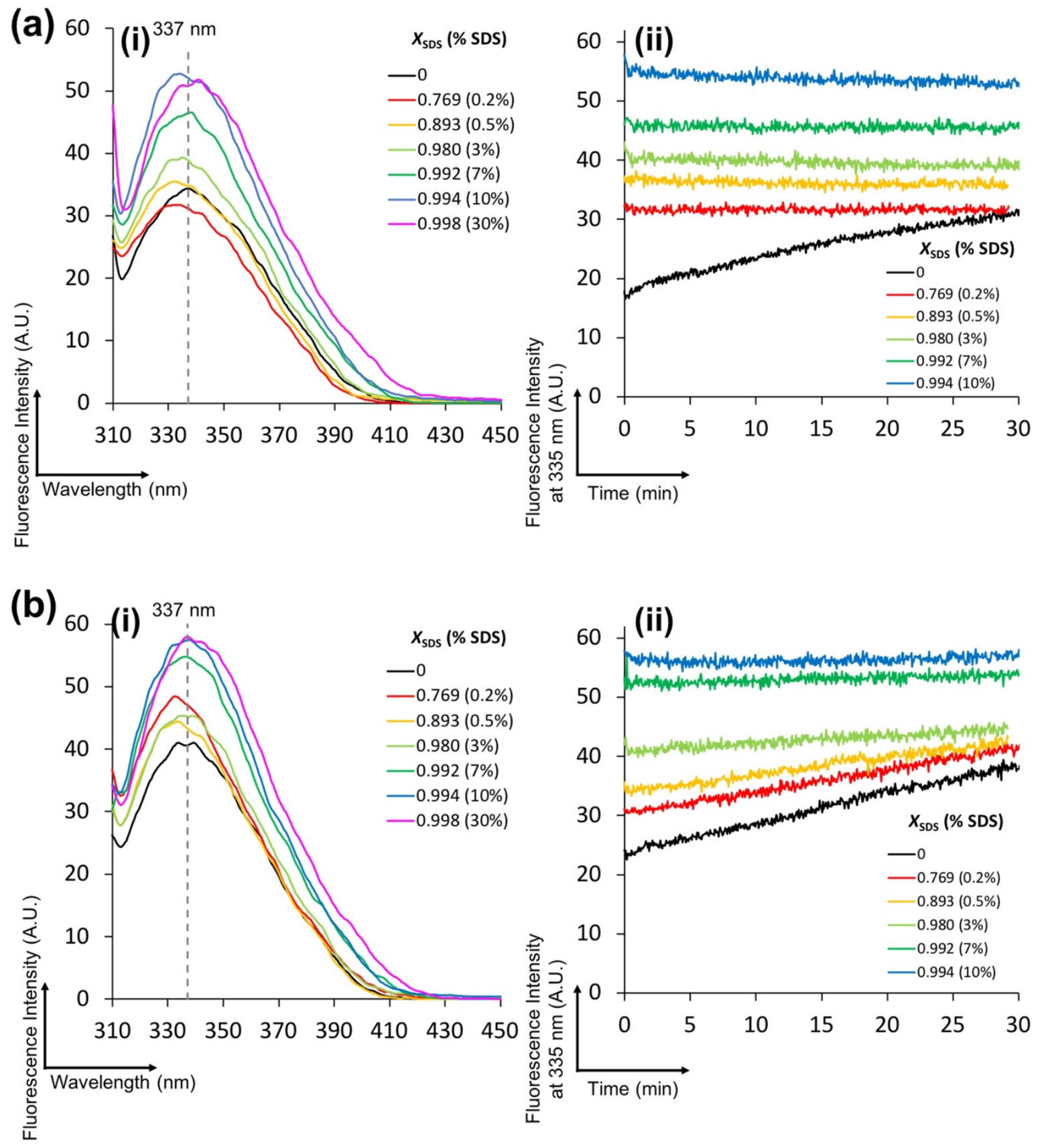

Figure S7. Emission spectra (a(i), $\mathbf{b}(\mathbf{i}))$ and time-courses (a(ii), $\mathbf{b}(\mathbf{i i}))$ of $7 \mu \mathrm{M}$ pSRII unfolded for $30 \mathrm{~min}$ in $0-0.998 X_{\mathrm{SDS}}(0-30 \% \mathrm{SDS})$ at $\mathrm{pH} 2.0$, in the absence (a) or presence (b) of $10 \mathrm{mM}$ hydroxylamine. Emission $\lambda_{\max }(337 \mathrm{~nm})$ of pSRII in 0\% SDS and 0\% SDS $+10 \mathrm{mM}$ hydroxylamine at pH 2.0 are shown as dotted lines. 
(a)

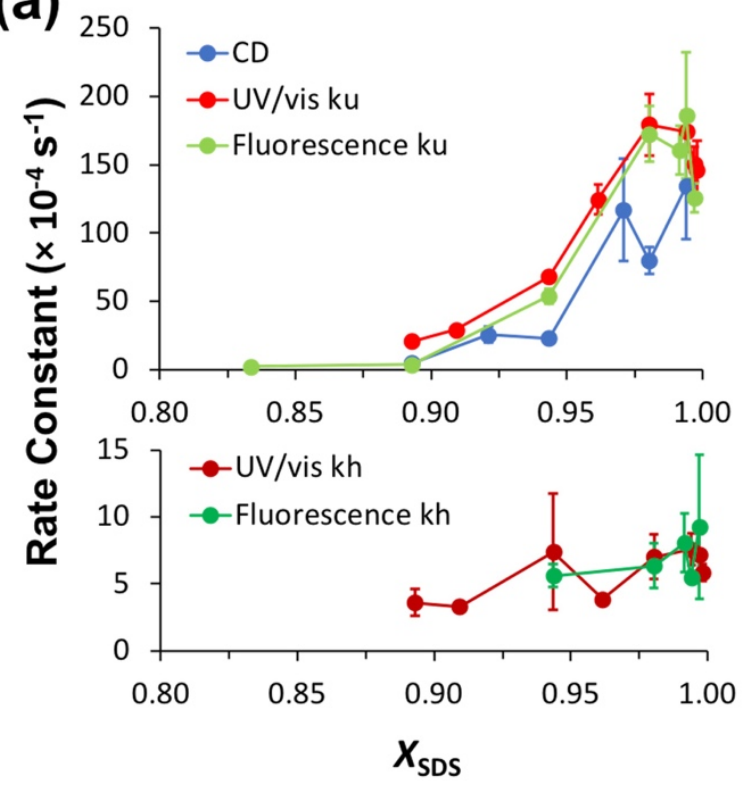

(b)

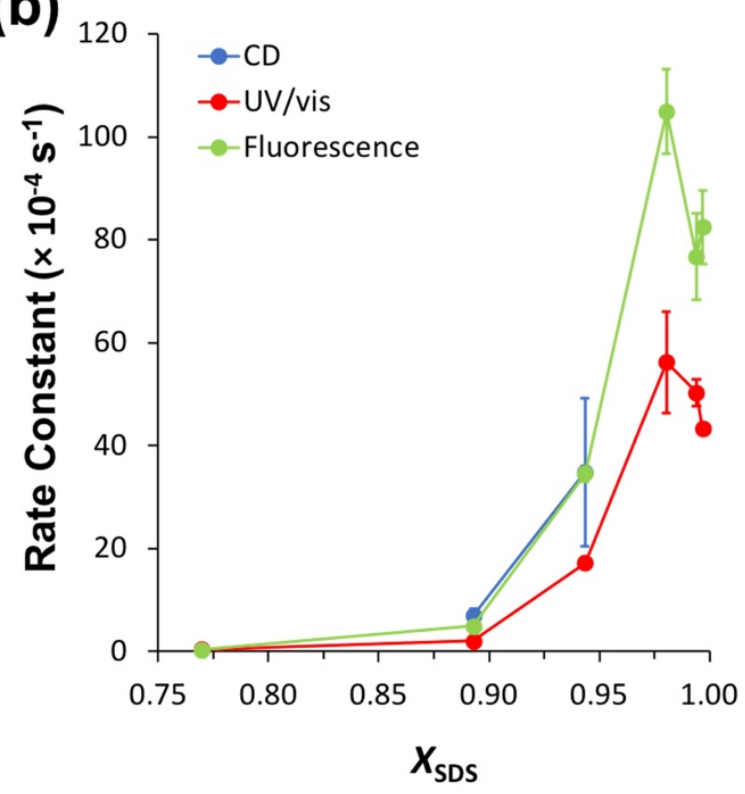

Figure S8. Dependence of rate constants of pSRII unfolding on $\boldsymbol{X}_{\text {SDS. }}$ (a) In the presence of SDS only (no hydroxylamine), two rate constants, $k_{\mathrm{u}}$ and $k_{\mathrm{h}}$, were extracted to describe protein unfolding and retinal Schiff base hydrolysis, respectively. (b) In the presence of $10 \mathrm{mM}$ hydroxylamine, only one rate constant was extracted. Error bars in both panels represent the standard deviation of 3 independent measurements. 

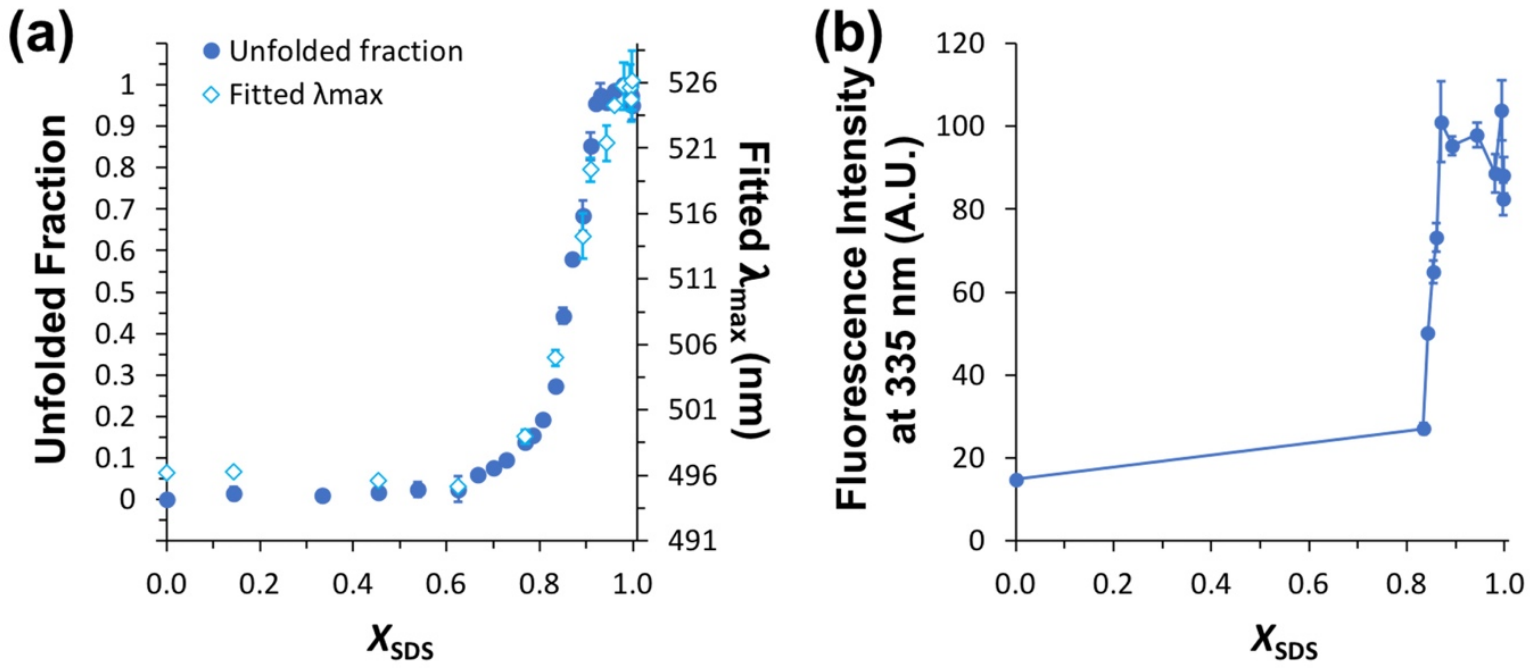

Figure S9. Comparison of the unfolding transition of pSRII in $0-0.998 X_{\text {SDS }}(0-30 \%$ SDS $)+0.1 \%$ c7-DHPC at pH 6.0 across different biophysical techniques. (a) SDS-induced equilibrium unfolding of pSRII monitored by UV/vis spectroscopy. $\lambda_{\max }$ of the retinal chromophore at $t=0 \mathrm{~min}$, obtained by non-linear least squares fitting, is also plotted against $X_{\mathrm{SDS}}$, showing that the chromophore $\lambda_{\max }$ is dependent on $X_{\text {SDS }}$. (b) Tryptophan fluorescence emission at $335 \mathrm{~nm}$. Error bars in all panels represent the standard deviation of 3 independent measurements. 

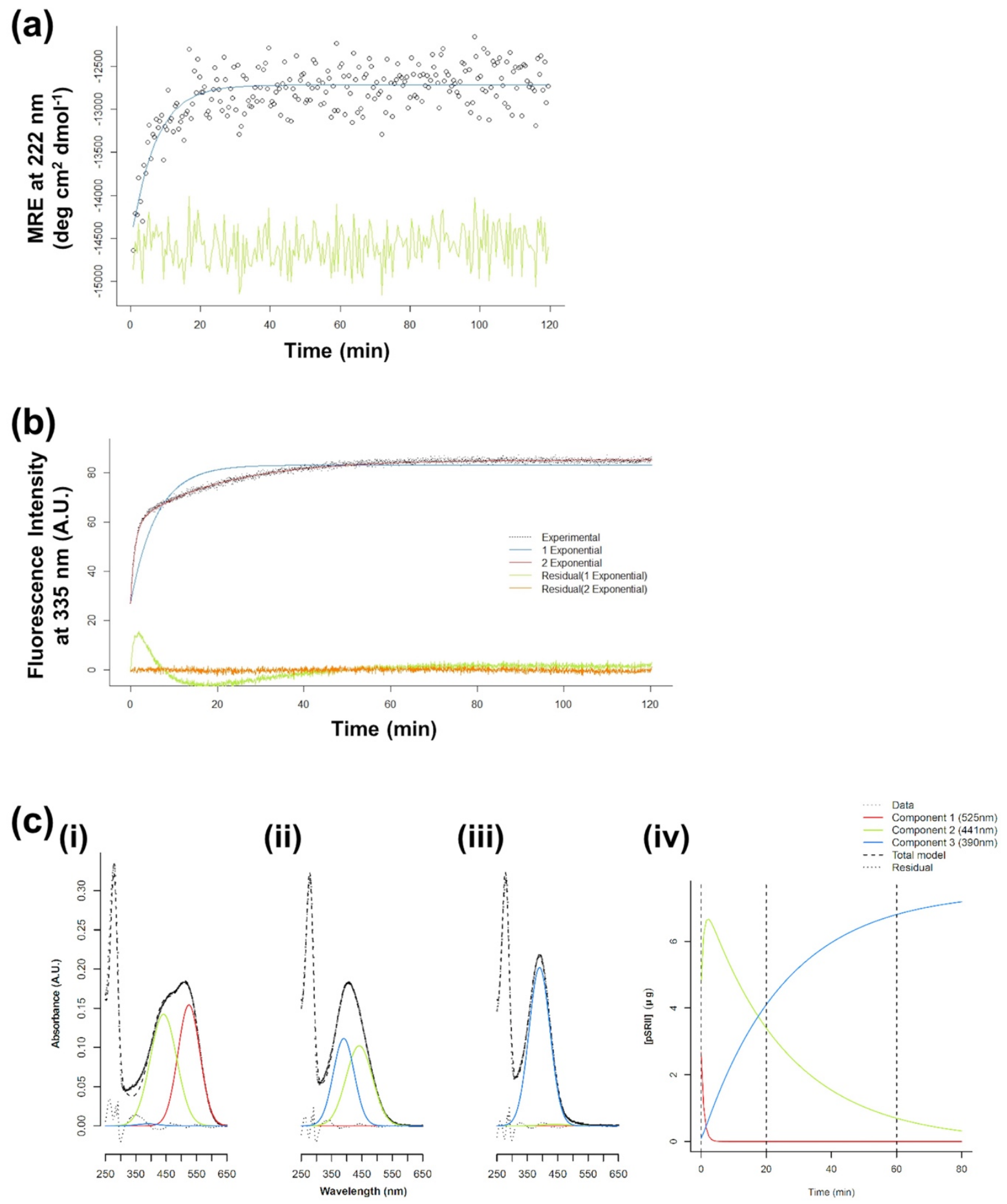

Figure S10. Examples of non-linear least squares fitting for time-courses recorded by (a) far-UV circular dichroism, (b) tryptophan fluorescence and (c) UV/vis spectroscopy. In panel c, time-resolved UV/vis spectra of pSRII unfolded in $0.980 X_{\text {SDS }}$ (3\% SDS) are shown for (i) $t=0$ min (dead-time $=25 \mathrm{~s}$ ), (ii) $t=20 \mathrm{~min}$, and (iii) $t=60 \mathrm{~min}$. Nonlinear least squares fitting was used to deconvolute the spectra into three spectral components (red, lime and blue lines). Goodness-of-fit can be assessed from the residual and the total model taken as sum of the three spectral components. The concentration of each component is plotted against time in panel c(iv). 
(a)

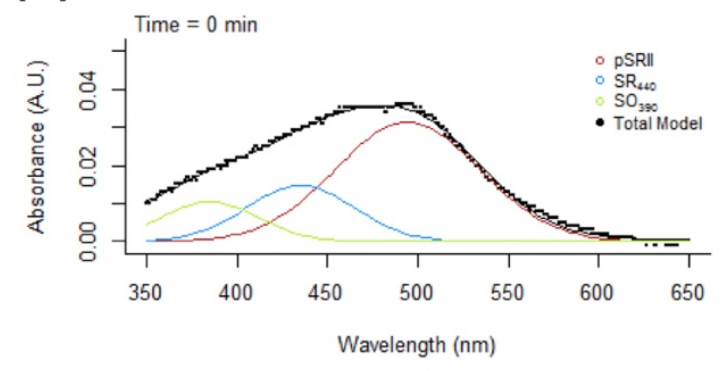

(b)

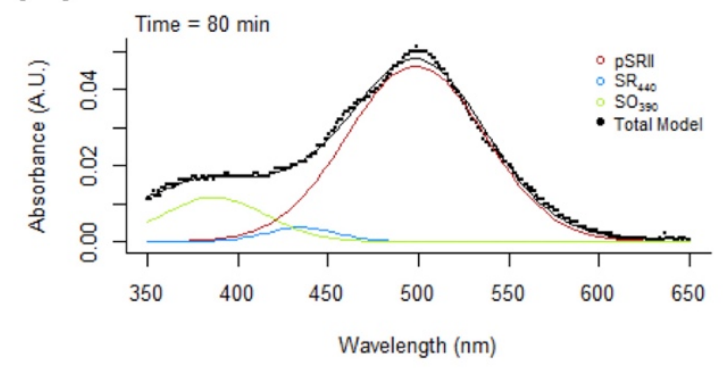

(d)

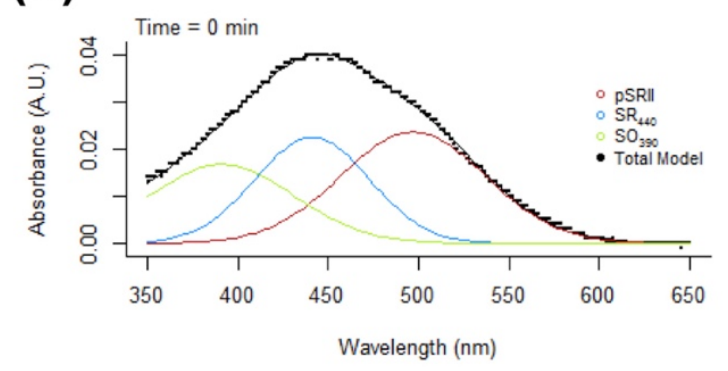

(e)

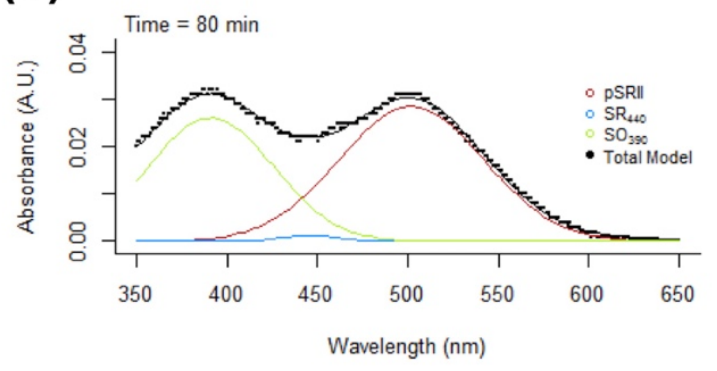

(c)

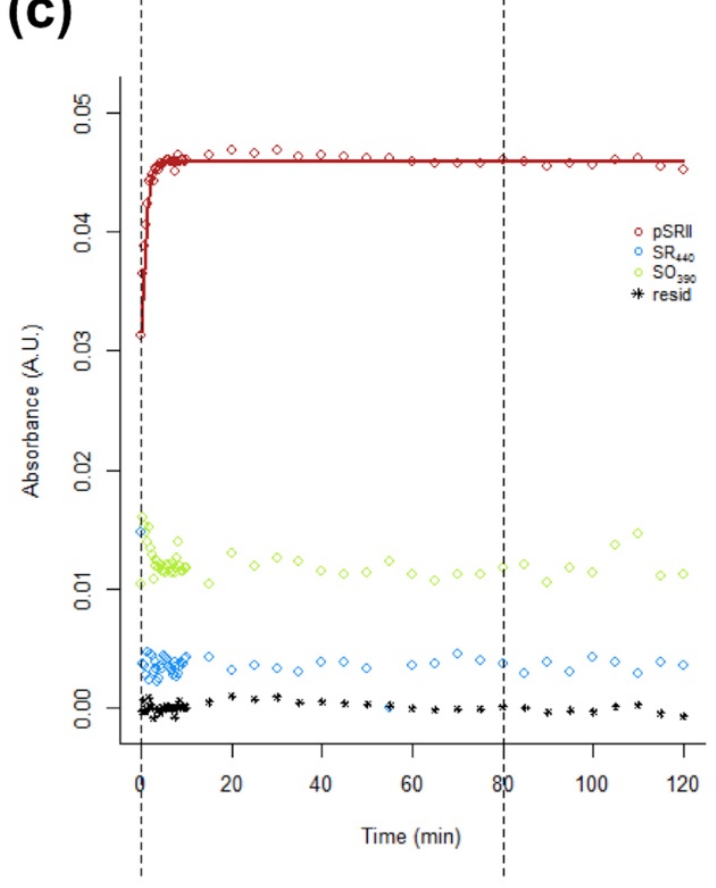

(f)

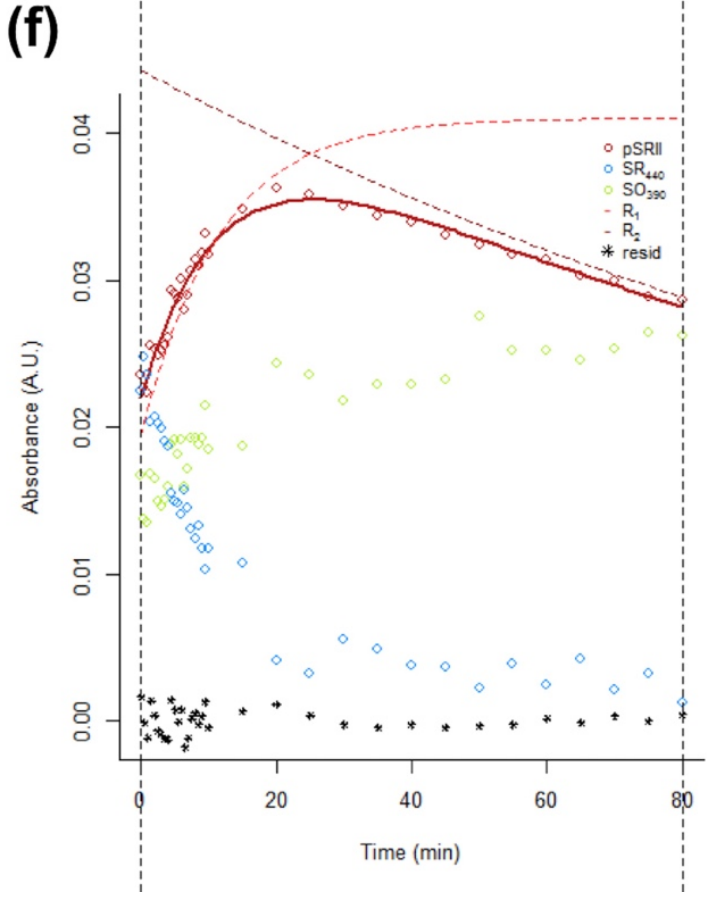

Figure S11: Examples of non-linear least squares fitting for UV/vis time-courses of refolding from $\mathrm{SR}_{440}$ in $\mathbf{0 . 7 2 3} X_{\text {SDS }}(\mathrm{a}-\mathrm{c})$ and $\mathbf{0 . 8 0 6} X_{\text {SDS }}(\mathrm{d}-\mathrm{f})$. Time-resolved UV/vis spectra of refolding from $\mathrm{SR}_{440}$ are shown for $t=0 \mathrm{~min}$ (dead-time $=20 \mathrm{~s})(\mathbf{a}, \mathbf{d})$ ), and $t=80 \mathrm{~min}(\mathbf{b}, \mathbf{f})$. The spectra were deconvoluted by non-linear least squares (NLS) fitting to yield three spectral components (red, lime and blue lines). Goodness of fit can be assessed from the total model taken as sum of the three spectral components. The absorbance of each spectral component is plotted in panels $\mathbf{c}$ and $\mathbf{f}$. Changes in absorbance at $\sim 500 \mathrm{~nm}$ during refolding in $0.723 X_{\text {SDS }}$ was fitted to a mono-exponential model to yield one rate constant for the recovery of the native chromophore. A double exponential model was used to describe refolding in $0.806 X_{\text {SDs }}$ to yield two rate constants $\left(R_{1}\right.$ and $\left.R_{2}\right)$ for the fast recovery of the native chromophore and slow decay to form $\mathrm{SO}_{390}$, respectively. 
(a)

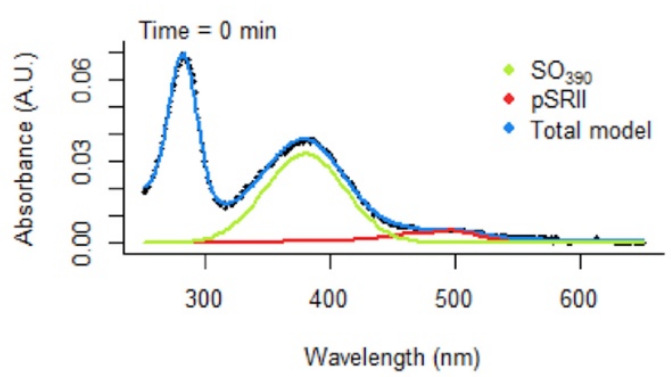

(b)

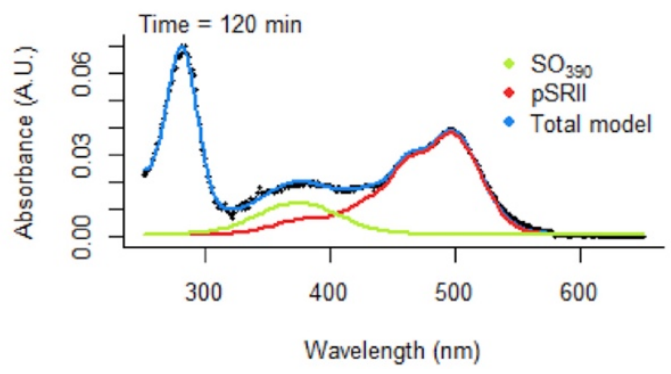

(c)

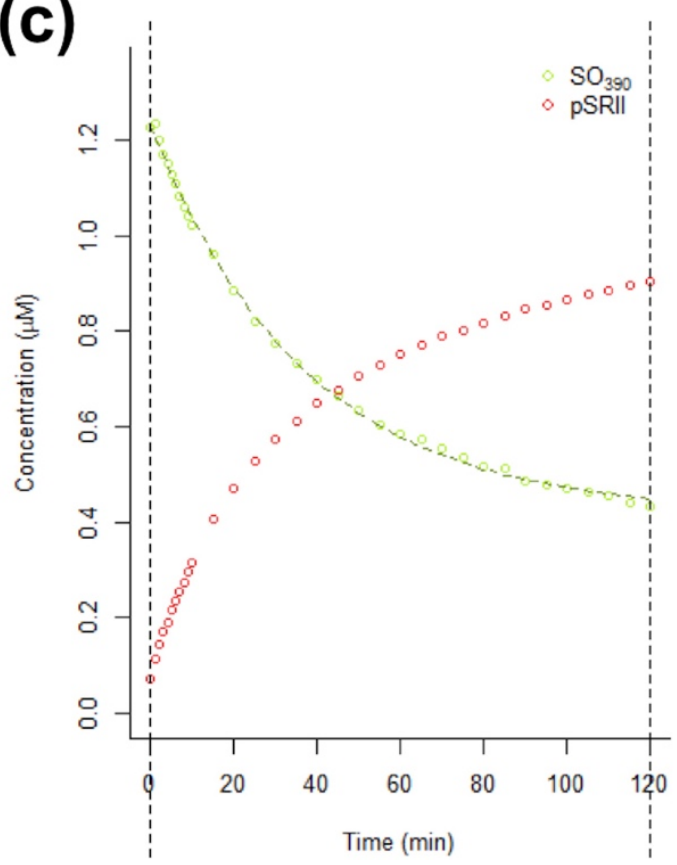

Figure S12: Example of non-linear least squares fitting for UV/vis time-courses of refolding from $\mathrm{SO}_{390}$ in $\mathbf{0 . 7 4 5} X_{\mathrm{SDS}}(\mathrm{a}-\mathrm{c})$ and $\mathbf{0 . 8 0 6} X_{\mathrm{SDS}}(\mathrm{d}-\mathrm{f})$. Time-resolved UV/vis spectra of refolding from $\mathrm{SR}_{390}$ are shown for $t=0 \mathrm{~min}$ (dead-time $=20 \mathrm{~s}$ ) (a), and $t=120 \mathrm{~min}(\mathbf{b})$. The spectra were deconvoluted by non-linear least squares (NLS) fitting to yield two spectral components (red and lime lines). Goodness of fit can be assessed from the total model taken as sum of the three spectral components. The absorbance of each spectral component is plotted in panel c. Changes in absorbance at $\sim 390 \mathrm{~nm}$ during refolding was fitted to a mono-exponential model to yield one apparent rate constant for the decay of $\mathrm{SO}_{390}$. 
(a)

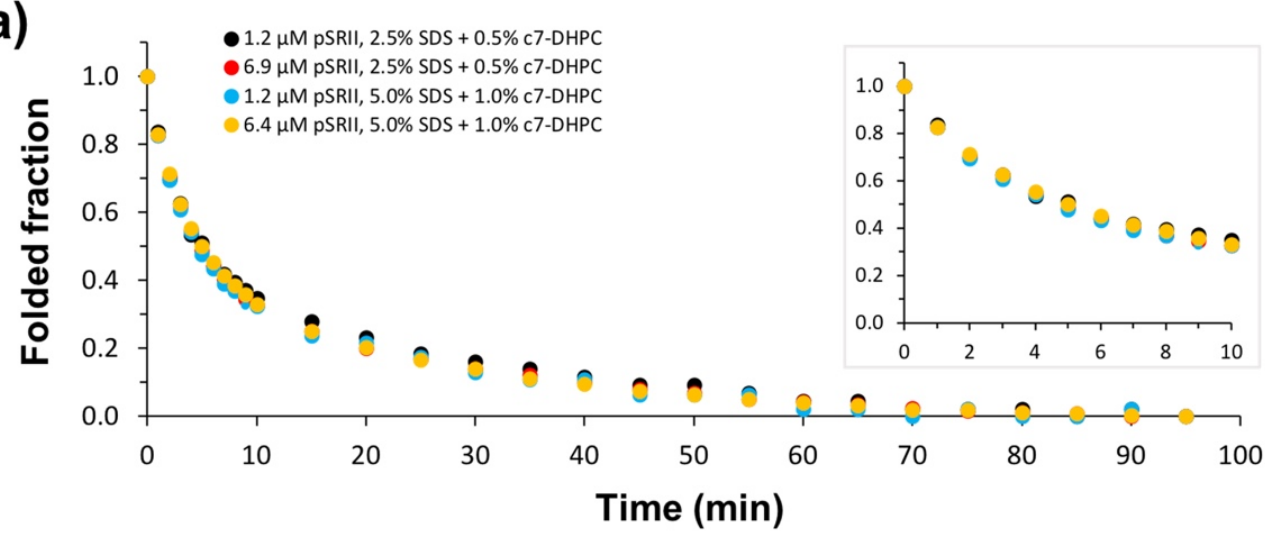

(b)

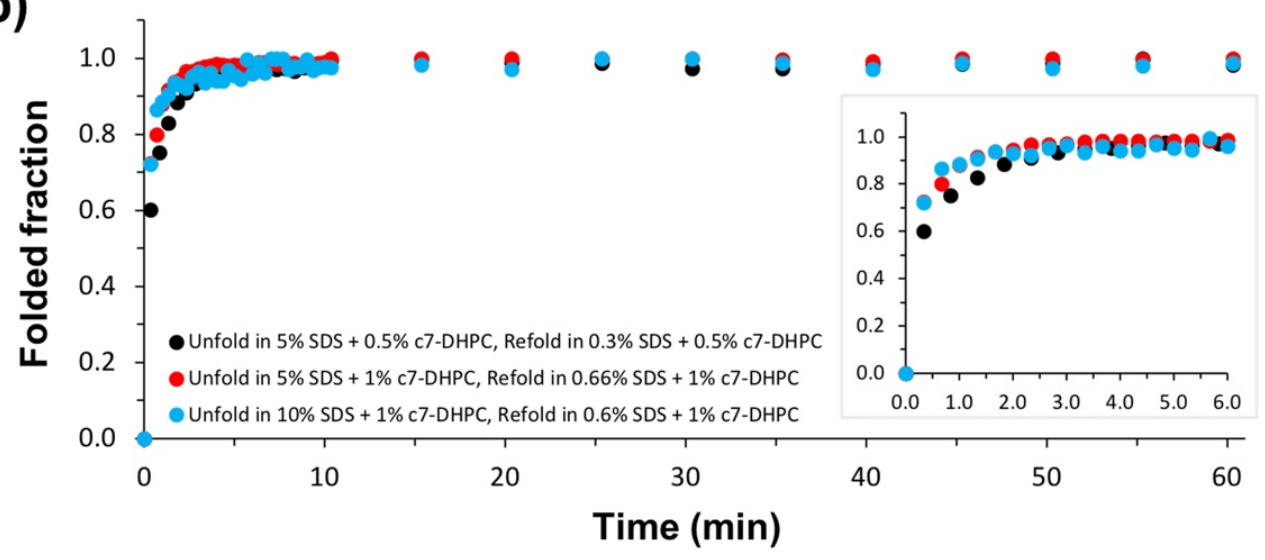

(c)

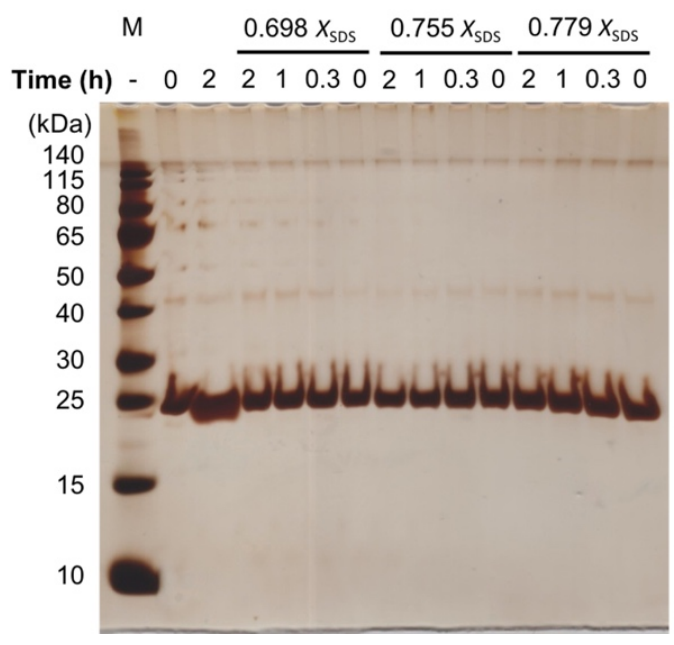

Figure S13. Time-courses for unfolding pSRII in $0.893 X_{S D S}(a)$ and refolding of $S_{440}$ in $0.698 X_{S D S}(b)$ at different protein concentrations and/or detergent concentrations. Normalised changes in $A_{498}$ (a) or nonlinear least squares fitted $A_{500}$ (b) are plotted against time, showing that unfolding and refolding rates are not dependent on protein concentration or detergent concentration. $1.2 \mu \mathrm{M}$ pSRII was used in refolding experiments shown in panel (b). Insets in both panels show expanded view of the earliest time points. (c) SDS-PAGE for refolding from $\mathrm{SR}_{440}$ for different durations $(0 \mathrm{~min}$, $20 \mathrm{~min}, 1 \mathrm{~h}, 2 \mathrm{~h}$ ) in the presence of different $X_{\mathrm{SDS}}$ in $0.5 \% \mathrm{c7}-\mathrm{DHPC}$, showing no evidence for the formation of stable aggregates. Lane 1, molecular weight marker; lanes 2-3, pSRII unfolded in 0.893 $X_{\mathrm{SDS}}$ for 0 min and $2 \mathrm{~h}$, respectively; lanes $4-15, \mathrm{pSRII}$ was first unfolded for $4 \mathrm{~min}$ in $0.893 X_{\mathrm{SDS}}$ to obtain $\mathrm{SR}_{440}$, followed by refolding for $0 \mathrm{~min}, 20 \mathrm{~min}, 1 \mathrm{~h}, 2 \mathrm{~h}$ in $0.698 X_{\text {SDS }}$ (lanes 4-7), $0.755 X_{\text {SDS }}$ (lanes 8-11) and $0.779 X_{\text {SDS }}$ (lanes 12-15). 

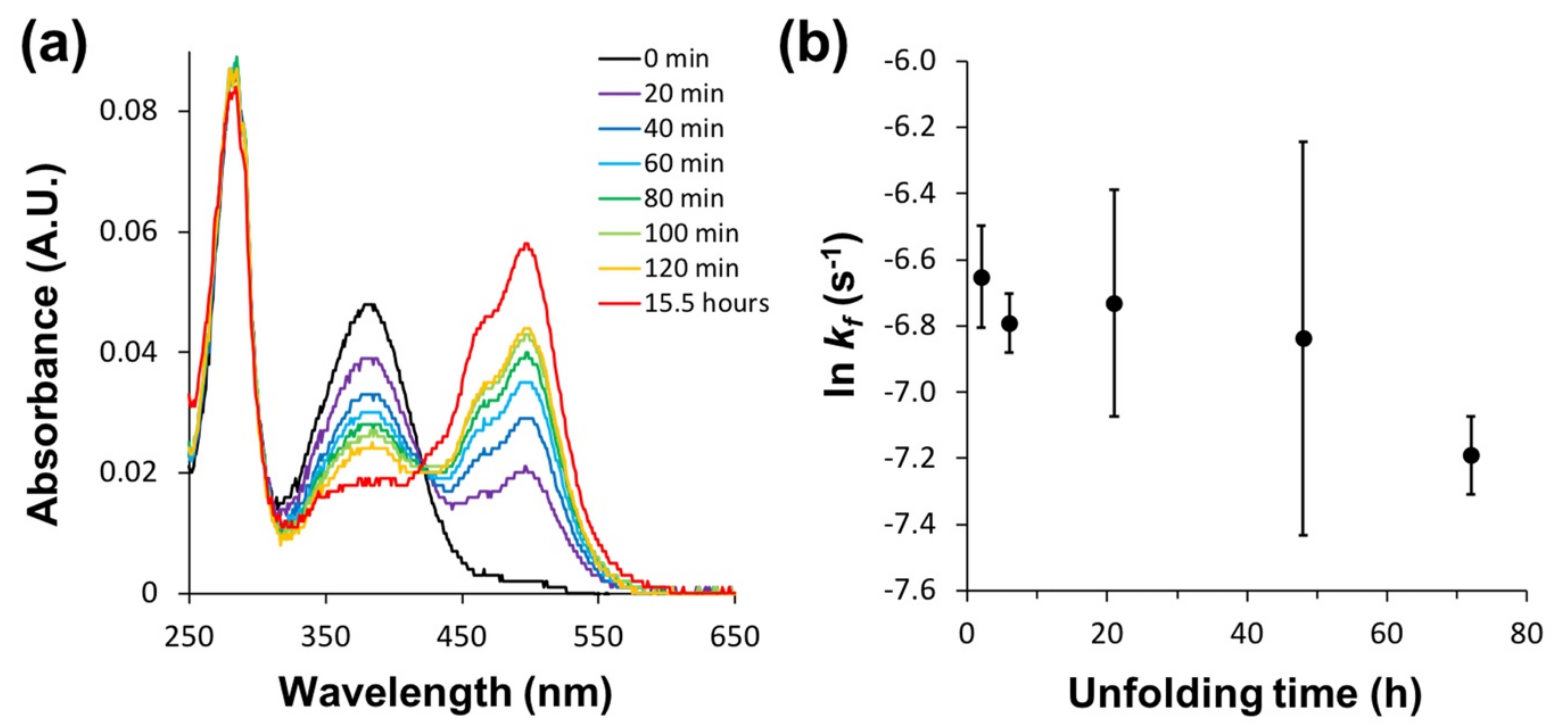

Figure S14. (a) Example time-course of refolding of $\mathrm{SO}_{390}$ in $0.480 \mathrm{X}_{\mathrm{SDS}}$ in $0.5 \% \mathrm{c} 7-\mathrm{DHPC}$, showing additional recovery of the native chromophore (red trace) after overnight refolding at room temperature. (b) The rate constant for refolding remains the same regardless of the time spent in the $\mathrm{SO}_{390}$ state. Error bars represent the standard deviation of 3 independent measurements. 
(a)

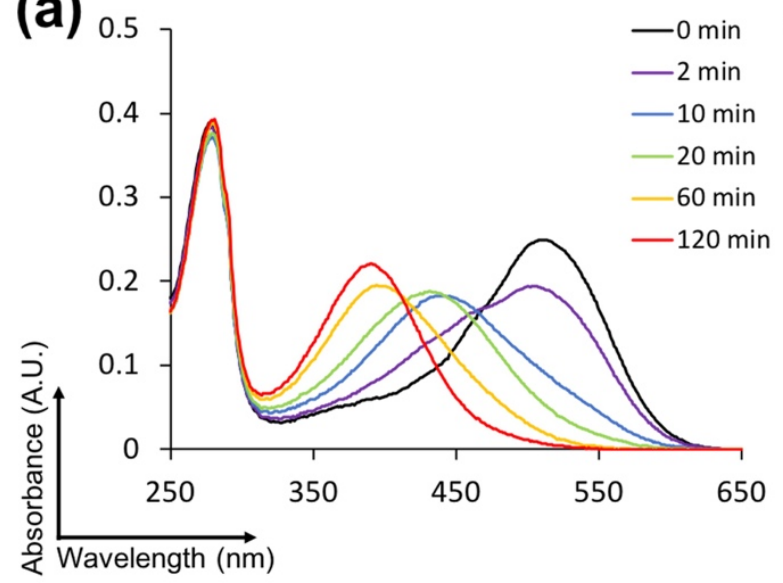

(b)

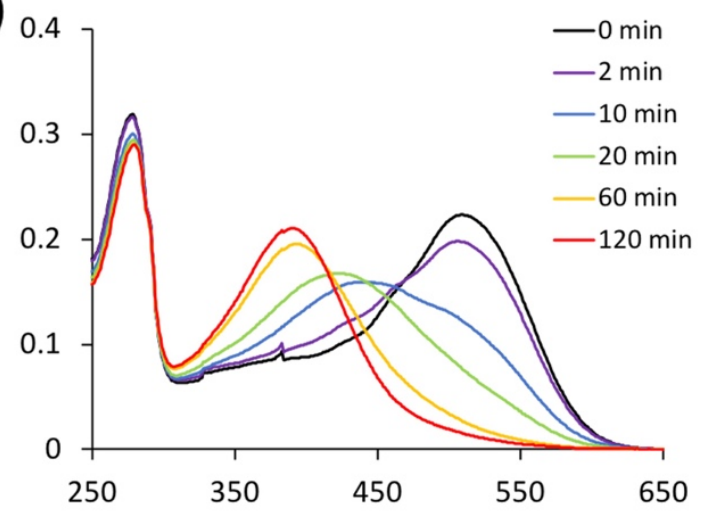

Figure S15. Time-resolved UV/vis spectra of pSRII unfolded in (a) $0.6 \%$ SDS + 0.1\% c7-DHPC (0.909 $\left.X_{\text {SDS }}\right)$ and (b) $0.3 \%$ SDS + 0.05\% DDM (0.914 $\left.X_{\text {SDS }}\right)$. 


\section{Supplementary Tables}

\begin{tabular}{|c|c|c|c|c|c|c|}
\hline & Denaturant & Example & Ref. & Absorbance & $\begin{array}{c}\text { Visual } \\
\text { Inspection }\end{array}$ & Conclusion \\
\hline \multirow{4}{*}{$\begin{array}{l}\text { Water-soluble } \\
\text { denaturants }\end{array}$} & Urea & Bovine rhodopsin & [7] & $\begin{array}{l}\text { No change } \\
\text { (Native-like } \\
\text { major peak at } \\
498 \mathrm{~nm} \text { with } \\
\text { side bands at } \\
460 \mathrm{~nm}, 420 \mathrm{~nm} \\
\text { and } 370 \mathrm{~nm} \text { ) }\end{array}$ & Orange; clear & $\begin{array}{l}\text { Does not } \\
\text { unfold }\end{array}$ \\
\hline & $\begin{array}{c}\text { Guanidine } \\
\text { hydrochloride }\end{array}$ & Bovine rhodopsin & [7] & No change & Orange; clear & $\begin{array}{l}\text { Does not } \\
\text { unfold }\end{array}$ \\
\hline & TFE & $\begin{array}{l}\text { KcsA potassium } \\
\text { channel }\end{array}$ & {$[8,9]$} & $\begin{array}{l}\text { N/A } \\
\text { (aggregation } \\
\text { observable by } \\
\text { eye) }\end{array}$ & $\begin{array}{l}\text { Pale yellow; } \\
\text { cloudy }\end{array}$ & $\begin{array}{l}\text { Unfolds } \\
\text { but } \\
\text { aggregates }\end{array}$ \\
\hline & TFA & Bovine rhodopsin & [7] & $\begin{array}{l}\text { Major peak at } \\
390 \mathrm{~nm} \text {; minor } \\
\text { peak at } 498 \mathrm{~nm}\end{array}$ & $\begin{array}{l}\text { Bright yellow; } \\
\text { clear }\end{array}$ & $\begin{array}{l}\text { Unfolds } \\
\text { with slow } \\
\text { kinetics }\end{array}$ \\
\hline \multirow[b]{2}{*}{$\begin{array}{c}\text { Anionic } \\
\text { detergents }\end{array}$} & SDS & $\begin{array}{l}\text { Bovine rhodopsin; } \\
\text { Bacteriorhodopsin }\end{array}$ & {$[7,10]$} & $\begin{array}{l}\text { Single peak at } \\
390 \mathrm{~nm}\end{array}$ & $\begin{array}{l}\text { Pale yellow; } \\
\text { clear }\end{array}$ & Unfolds \\
\hline & $\mathrm{N}$-lauroylsarcosine & $\begin{array}{l}\text { Olfactory receptor } \\
\text { OR5; Peripheral } \\
\text { myelin protein } 22 \\
\text { (PMP22) }\end{array}$ & {$[11,12]$} & No change & Orange; clear & $\begin{array}{l}\text { Does not } \\
\text { unfold }\end{array}$ \\
\hline $\begin{array}{l}\text { Cationic } \\
\text { detergent }\end{array}$ & CTAB & $\begin{array}{l}\text { Cytochrome c } \\
\text { oxidase }\end{array}$ & [13] & $\begin{array}{l}\text { Major peak at } \\
390 \mathrm{~nm} \text {; minor } \\
\text { peak at } 498 \mathrm{~nm}\end{array}$ & $\begin{array}{l}\text { Pale yellow; } \\
\text { clear }\end{array}$ & $\begin{array}{l}\text { Unfolds } \\
\text { with slow } \\
\text { kinetics }\end{array}$ \\
\hline $\begin{array}{l}\text { Zwitterionic } \\
\text { detergent }\end{array}$ & LDAO & $\begin{array}{l}\text { Prokaryotic and } \\
\text { eukaryotic } \\
\text { transporters }\end{array}$ & [14] & $\begin{array}{l}\text { Major peak at } \\
498 \mathrm{~nm} \text {; minor } \\
\text { peak at } 390 \mathrm{~nm}\end{array}$ & Yellow; clear & $\begin{array}{l}\text { Unfolds } \\
\text { with very } \\
\text { slow } \\
\text { kinetics }\end{array}$ \\
\hline \multirow{2}{*}{$\begin{array}{l}\text { Non-ionic } \\
\text { detergents }\end{array}$} & Triton X-100 & 5- $\mathrm{HT}_{1 \mathrm{~A}}$ receptor & [15] & No change & Orange; clear & $\begin{array}{l}\text { Does not } \\
\text { unfold }\end{array}$ \\
\hline & Tween-20 & $\begin{array}{l}\text { Membrane protein } \\
\text { extraction }\end{array}$ & {$[16]$} & No change & Orange; clear & $\begin{array}{l}\text { Does not } \\
\text { unfold }\end{array}$ \\
\hline
\end{tabular}

Table S1. Effects of different denaturants on pSRII after a 1-day incubation at room temperature. TFE, 2,2,2-trifluoroethanol; TFA, trifluoroacetic acid; SDS, sodium dodecyl sulphate; CTAB, cetyltrimethylammonium bromide; LDAO, lauryldimethylamine-N-oxide; Triton X-100, polyoxyethylene octyl phenyl ether; Tween-20, polyoxyethylene (20) sorbitan monolaurate. 


\begin{tabular}{|c|c|c|c|l|}
\hline Additive/Buffer & Final Conc. & Ref. & Aggregation after 1 day & \multicolumn{1}{|c|}{ Conclusion } \\
\hline $\mathrm{NaCl}$ & $0-500 \mathrm{mM}$ & {$[1]$} & Yes & $\begin{array}{l}\text { More oligomeric } \\
\text { species than in the } \\
\text { absence of additive }\end{array}$ \\
\hline PEG-8000 & $0-30 \mathrm{mg} / \mathrm{ml}$ & {$[1]$} & Yes & $\begin{array}{l}\text { More oligomeric } \\
\text { species than in the } \\
\text { absence of additive }\end{array}$ \\
\hline LDAO & $0.18 \%(\mathrm{w} / \mathrm{v})$ & {$[17]$} & Yes & $\begin{array}{l}\text { Similar amount of } \\
\text { oligomeric species as } \\
\text { in the absence of } \\
\text { additive }\end{array}$ \\
\hline NDSB-201 & $1 \mathrm{M}$ & {$[1]$} & Yes & $\begin{array}{l}\text { More oligomeric } \\
\text { species than in the } \\
\text { absence of additive }\end{array}$ \\
\hline NDSB-256 & $1 \mathrm{M}$ & {$[1]$} & Yes & $\begin{array}{l}\text { More oligomeric } \\
\text { species than in the } \\
\text { absence of additive }\end{array}$ \\
\hline L-Arginine & $0.5 \mathrm{M}$ & {$[1]$} & No & $\begin{array}{l}\text { pSRII did not unfold } \\
\text { (see Fig. S2d) }\end{array}$ \\
\hline Urea & $0-8 \mathrm{M}$ & {$[7]$} & Yes & $\begin{array}{l}\text { More oligomeric } \\
\text { species than in the } \\
\text { absence of additive }\end{array}$ \\
\hline $\begin{array}{c}\text { Sodium } \\
\text { phosphate, }\end{array}$ & $50 \mathrm{mM}$ & {$[21]$} & & $\begin{array}{l}\text { Amount of } \\
\text { oligomerisation } \\
\text { depends on pH or } \\
\text { [TFA] }\end{array}$ \\
\hline TFA & $0-200 \mathrm{mM}$ & {$[18-20]$} & Yes & $\begin{array}{l}\text { Less oligomeric species } \\
\text { than in the absence of } \\
\text { additive }\end{array}$ \\
\hline
\end{tabular}

Table S2. Aggregation of pSRII unfolded for 1 day at room temperature in 3\% SDS + different additives/buffer conditions. 


\begin{tabular}{|c|c|c|c|c|c|c|c|}
\hline pH & $\boldsymbol{X}_{\text {SDS }}$ & $\mathbf{H}(\mathbf{r})$ & $\mathbf{H}(\mathbf{d})$ & $\mathbf{S}(\mathbf{r})$ & $\mathbf{S}(\mathbf{d})$ & $\mathbf{T}$ & $\mathbf{U}$ \\
\hline \multirow{5}{*}{$\mathrm{pH} 6.0$} & 0 & $47.3 \pm 1.3 \%$ & $17.0 \pm 0.7 \%$ & $0.6 \pm 0.1 \%$ & $3.1 \pm 0.4 \%$ & $10.4 \pm 0.6 \%$ & $21.6 \pm 0.3 \%$ \\
\cline { 2 - 8 } & 0.625 & $47.5 \pm 1.2 \%$ & $17.4 \pm 0.3 \%$ & $0.4 \pm 0.2 \%$ & $2.7 \pm 0.2 \%$ & $10.6 \pm 0.5 \%$ & $21.4 \pm 0.4 \%$ \\
\cline { 2 - 8 } & 0.834 & $45.6 \pm 0.7 \%$ & $17.4 \pm 0.4 \%$ & $0.8 \pm 0.3 \%$ & $3.3 \pm 0.2 \%$ & $11.6 \pm 0.3 \%$ & $21.3 \pm 0.2 \%$ \\
\cline { 2 - 8 } & 0.893 & $38.1 \pm 0.4 \%$ & $18.6 \pm 0.2 \%$ & $2.2 \pm 0.2 \%$ & $3.9 \pm 0.1 \%$ & $13.9 \pm 0.3 \%$ & $23.2 \pm 0.2 \%$ \\
\cline { 2 - 8 } & 0.943 & $38.7 \pm 0.3 \%$ & $19.0 \pm 0.1 \%$ & $1.7 \pm 0.2 \%$ & $3.9 \pm 0.1 \%$ & $13.6 \pm 0.2 \%$ & $23.0 \pm 0.1 \%$ \\
\cline { 2 - 8 } & 0.980 & $38.9 \pm 1.8 \%$ & $19.1 \pm 0.8 \%$ & $1.4 \pm 0.4 \%$ & $4.0 \pm 0.2 \%$ & $13.6 \pm 0.5 \%$ & $23.0 \pm 0.6 \%$ \\
\cline { 2 - 8 } & 0.994 & $40.7 \pm 2.6 \%$ & $20.3 \pm 2.1 \%$ & $2.4 \pm 1.9 \%$ & $3.0 \pm 1.0 \%$ & $8.9 \pm 7.8 \%$ & $24.5 \pm 3.3 \%$ \\
\cline { 2 - 8 } & 0.997 & $41.1 \pm 0.5 \%$ & $20.0 \pm 0.7 \%$ & $1.5 \pm 1.0 \%$ & $3.6 \pm 0.3 \%$ & $12.1 \pm 1.7 \%$ & $21.7 \pm 2.8 \%$ \\
\cline { 2 - 8 } & 0.998 & $40.3 \pm 1.4 \%$ & $19.6 \pm 0.4 \%$ & $0.8 \pm 0.7 \%$ & $3.6 \pm 0.3 \%$ & $13.1 \pm 0.4 \%$ & $22.5 \pm 0.6 \%$ \\
\hline \multirow{5}{*}{ pH 2.0 } & 0 & $46.0 \pm 1.4 \%$ & $17.3 \pm 0.7 \%$ & $1.1 \pm 0.2 \%$ & $3.1 \pm 0.4 \%$ & $11.5 \pm 0.6 \%$ & $21.0 \pm 1.2 \%$ \\
\cline { 2 - 8 } & 0.769 & $47.6 \pm 1.0 \%$ & $18.6 \pm 0.4 \%$ & $0.8 \pm 0.4 \%$ & $2.8 \pm 0.3 \%$ & $10.9 \pm 0.4 \%$ & $19.3 \pm 0.3 \%$ \\
\cline { 2 - 8 } & 0.893 & $42.2 \pm 1.3 \%$ & $18.6 \pm 0.6 \%$ & $1.7 \pm 0.4 \%$ & $3.4 \pm 0.2 \%$ & $12.7 \pm 0.3 \%$ & $21.4 \pm 0.5 \%$ \\
\cline { 2 - 8 } & 0.980 & $37.8 \pm 3.3 \%$ & $17.7 \pm 0.2 \%$ & $3.0 \pm 1.8 \%$ & $4.5 \pm 0.2 \%$ & $13.7 \pm 0.9 \%$ & $23.2 \pm 0.7 \%$ \\
\cline { 2 - 7 } & 0.994 & $39.6 \pm 0.6 \%$ & $18.3 \pm 0.3 \%$ & $1.9 \pm 0.4 \%$ & $4.1 \pm 0.2 \%$ & $13.4 \pm 0.1 \%$ & $22.7 \pm 0.1 \%$ \\
\cline { 2 - 7 } & 0.998 & $41.2 \pm 0.7 \%$ & $19.5 \pm 1.6 \%$ & $1.4 \pm 0.5 \%$ & $3.5 \pm 0.8 \%$ & $11.3 \pm 2.4 \%$ & $23.0 \pm 0.9 \%$ \\
\hline
\end{tabular}

Table S3. Deconvolution of CD spectra for pSRII unfolded for 2 hours in different $X_{\text {SDs }}$ at $\mathrm{pH} 6.0$ and for $30 \mathrm{~min}$ at $\mathrm{pH}$ 2.0. Deconvolution was performed using CDPro with CONTINLL algorithm and basis set no. 10 (contains soluble and membrane proteins). $H(r)$, regular helix; $H(d)$, distorted helix; $S(r)$, regular strand; S(d), distorted strand; T, turns; $U$, unordered. Errors are reported as standard deviations of 3 independent measurements. 


\section{References:}

[1] H. Hamada, T. Arakawa, K. Shiraki, Effect of additives on protein aggregation., Curr. Pharm. Biotechnol. 10 (2009) 400-407. doi:10.2174/138920109788488941.

[2] G.Q. Chen, E. Gouaux, Probing the folding and unfolding of wild-type and mutant forms of bacteriorhodopsin in micellar solutions: Evaluation of reversible unfolding conditions, Biochemistry. 38 (1999) 15380-15387. doi:10.1021/bi9909039.

[3] A. Fersht, Structure and mechanism in protein science: A guide to enzyme catalysis and protein folding., 1999. doi:10.1016/S0307-4412(99)00114-4.

[4] M. Silow, M. Oliveberg, Transient aggregates in protein folding are easily mistaken for folding intermediates, Proc. Natl. Acad. Sci. 94 (1997) 6084-6086. doi:10.1073/pnas.94.12.6084.

[5] M. Wyckoff, D. Rodbard, A. Chrambach, Polyacrylamide gel electrophoresis in sodium dodecyl sulfate-containing buffers using multiphasic buffer systems: Properties of the stack, valid Rf- measurement, and optimized procedure, Anal. Biochem. 78 (1977) 459-482. doi:10.1016/0003-2697(77)90107-5.

[6] M. Samsó, J. -R Daban, S. Hansen, G.R. Jones, Evidence for Sodium Dodecyl Sulfate/Protein Complexes Adopting a Necklace Structure, Eur. J. Biochem. 232 (1995) 818-824. doi:10.1111/j.1432-1033.1995.0818a.x.

[7] A. Dutta, K.C. Tirupula, U. Alexiev, J. Klein-Seetharaman, Characterization of membrane protein non-native states. 1. Extent of unfolding and aggregation of rhodopsin in the presence of chemical denaturants, Biochemistry. 49 (2010) 6317-6328. doi:10.1021/bi100338e.

[8] F.N. Barrera, M.L. Renart, M.L. Molina, J.A. Poveda, J.A. Encinar, A.M. Fernández, J.L. Neira, J.M. González-Ros, Unfolding and refolding in vitro of a tetrameric, $\alpha$-helical membrane protein: The prokaryotic potassium channel KcsA, Biochemistry. 44 (2005) 14344-14352. doi:10.1021/bi050845t.

[9] F.I. Valiyaveetil, Y. Zhou, R. MacKinnon, Lipids in the structure, folding, and function of the KcsA K+ channel, Biochemistry. 41 (2002) 10771-10777. doi:10.1021/bi026215y.

[10] E. London, H.G. Khorana, Denaturation and renaturation of bacteriorhodopsin in detergents and lipid-detergent mixtures., J. Biol. Chem. 257 (1982) 7003-7011.

[11] H. Kiefer, J. Krieger, J.D. Olszewski, G. Von Heijne, G.D. Prestwich, H. Breer, Expression of an olfactory receptor in Escherichia coli: Purification, reconstitution, and ligand binding, Biochemistry. 35 (1996) 16077-16084. doi:10.1021/bi9612069.

[12] J.K. Myers, C.K. Mobley, C.R. Sanders, The peripheral neuropathy-linked Trembler and Trembler-J mutant forms of peripheral myelin protein 22 are folding-destabilized, Biochemistry. 47 (2008) 10620-10629. doi:10.1021/bi801157p.

[13] T.K. Das, S. Mazumdar, Unfolding pathway of cytochrome c oxidase induced by ionic surfactants: Circular dichroism and picosecond time-resolved fluorescence studies, Proc. Indian Acad. Sci. Chem. Sci. 110 (1998) 479-490.

https://www.scopus.com/inward/record.uri?eid=2-s2.0-

0032184137\&partnerID=40\&md5=93d69c2fd48c0860867826ee110350ac.

[14] Y. Sonoda, S. Newstead, N.J. Hu, Y. Alguel, E. Nji, K. Beis, S. Yashiro, C. Lee, J. Leung, A.D. Cameron, B. Byrne, S. Iwata, D. Drew, Benchmarking membrane protein detergent stability for improving throughput of high-resolution x-ray structures, Structure. 19 (2011) 17-25. 
doi:10.1016/j.str.2010.12.001.

[15] S. Kalipatnapu, A. Chattopadhyay, Membrane protein solubilization: Recent advances and challenges in solubilization of serotonin1A receptors, IUBMB Life. 57 (2005) 505-512. doi:10.1080/15216540500167237.

[16] B.T. Arachea, Z. Sun, N. Potente, R. Malik, D. Isailovic, R.E. Viola, Detergent selection for enhanced extraction of membrane proteins, Protein Expr. Purif. 86 (2012) 12-20. doi:10.1016/j.pep.2012.08.016.

[17] A. Gautier, Structure determination of the seven-helix transmembrane receptor sensory rhodopsin II by solution NMR spectroscopy (Ph.D. thesis), University of Cambridge, 2009.

[18] K.S. Huang, H. Bayley, M.J. Liao, E. London, H.G. Khorana, Refolding of an integral membrane protein. Denaturation, renaturation, and reconstitution of intact bacteriorhodopsin and two proteolytic fragments., J. Biol. Chem. 256 (1981) 3802-3809.

[19] M.G. Zagorski, J. Yang, H. Shao, K. Ma, H. Zeng, A. Hong, Methodological and chemical factors affecting amyloid $\beta$ peptide amyloidogenicity, Methods Enzymol. 309 (1999) 189-204. doi:10.1016/S0076-6879(99)09015-1.

[20] S. Chen, R. Wetzel, Solubilization and disaggregation of polyglutamine peptides, Protein Sci. 10 (2001) 887-891. doi:10.1110/ps.42301.folding.

[21] E.Y. Chi, S. Krishnan, T.W. Randolph, J.F. Carpenter, Physical stability of proteins in aqueous solution: Mechanism and driving forces in nonnative protein aggregation, Pharm. Res. 20 (2003) 1325-1336. doi:10.1023/A:1025771421906. 


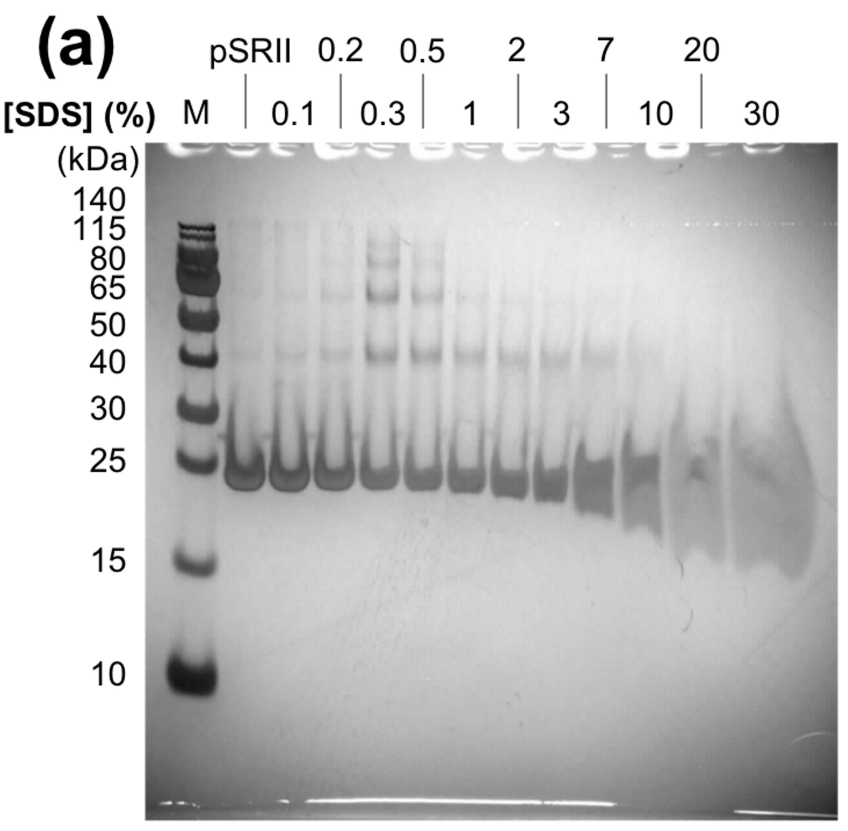

(c)

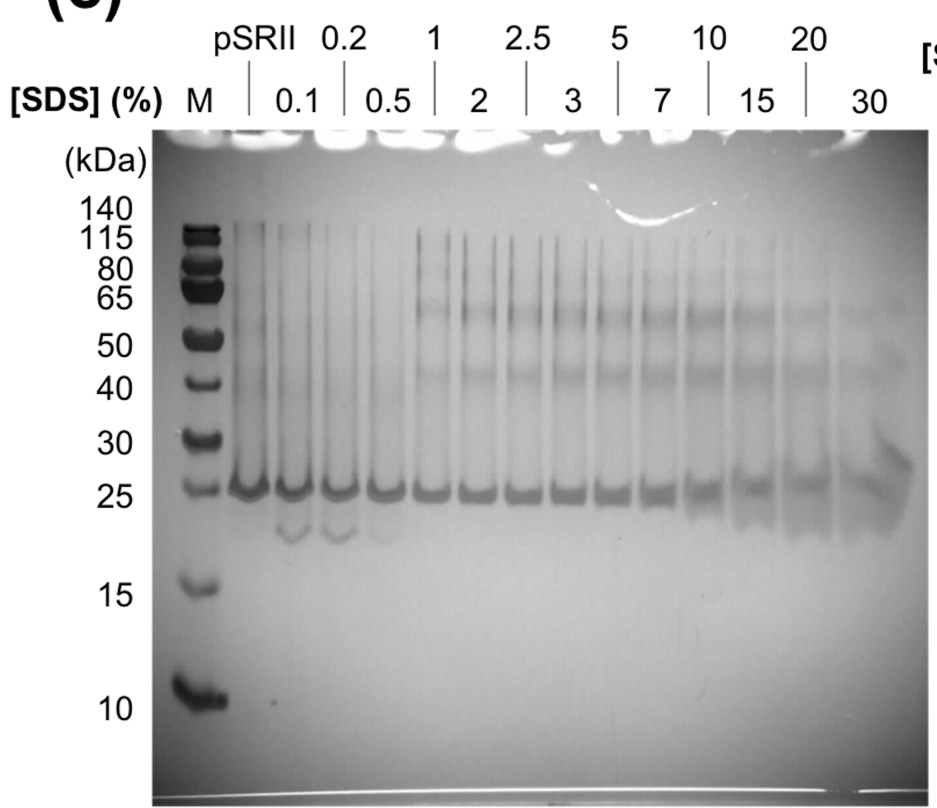

(e)

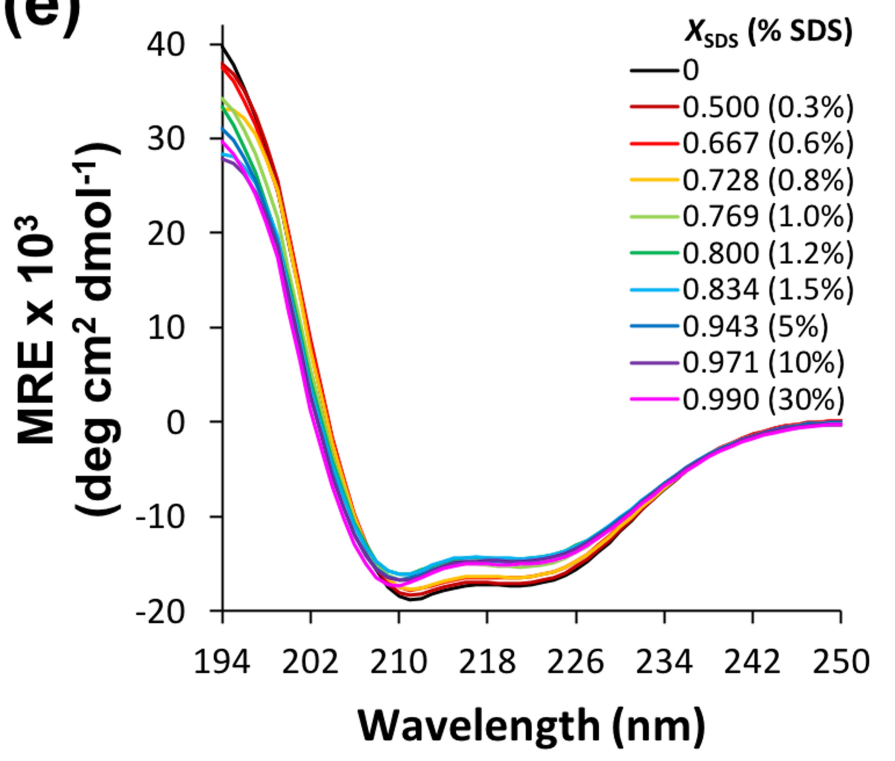

(b)

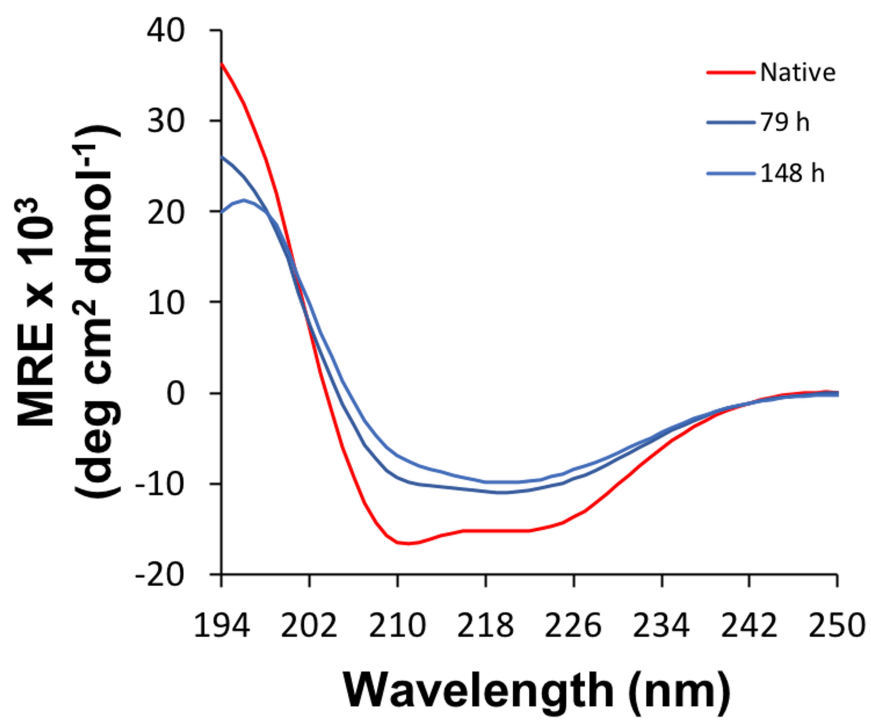

(d) $\begin{gathered}\text { [SDS] (\%) } \\ \text { Time (h) }\end{gathered}$
0 $\quad M \frac{0 \% \text { SDS }}{022472} \frac{2.5 \% \text { SDS }}{02244872} \frac{15 \% \text { SDS }}{022244872}$ $(\mathrm{kDa})$
140
115
80
65
50
40
30
25


15

(f)

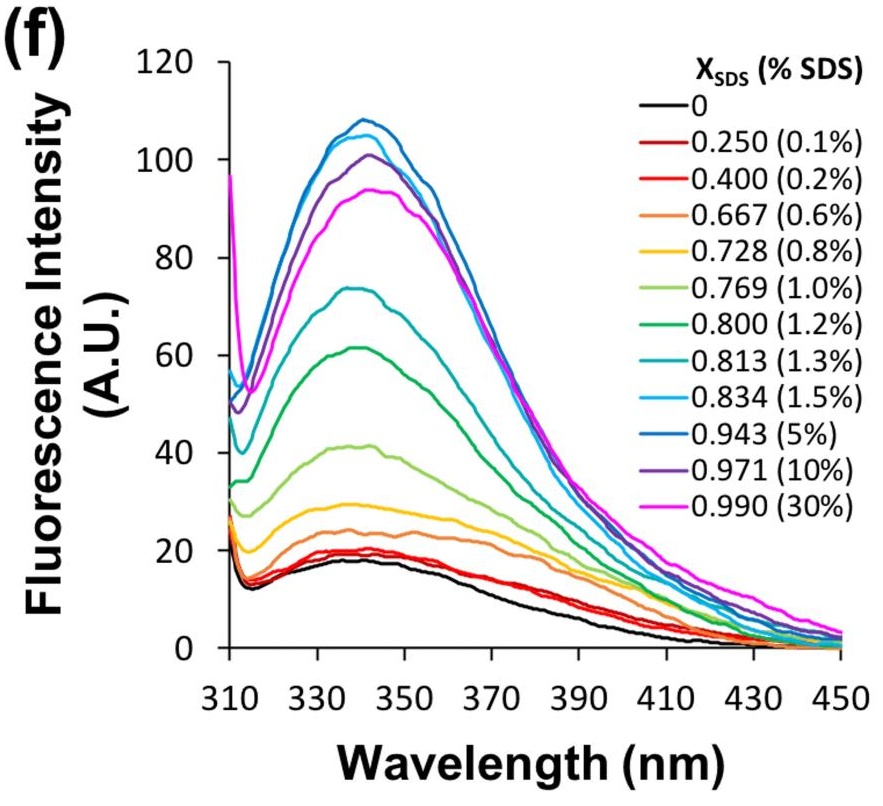


(a)

(b)

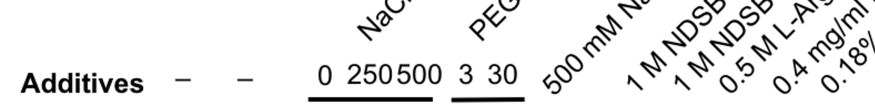

(kDa)

140
115

115
80

65

50

40

30

25

15

10
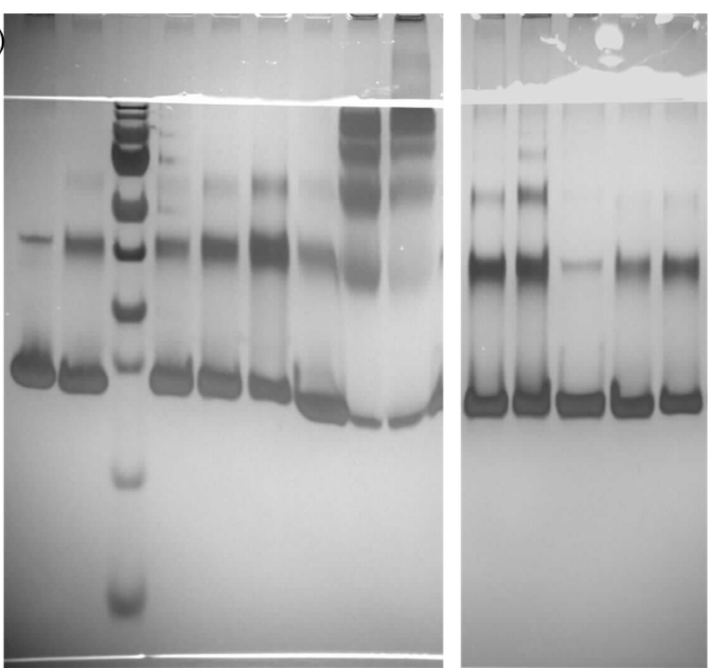

(c)

TFA \& Final pH

$3 \%$ SDS $\mathrm{M}-++++++$

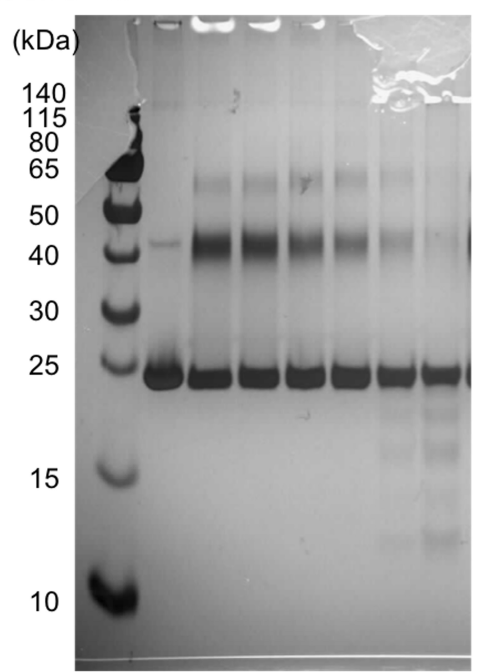

(d)

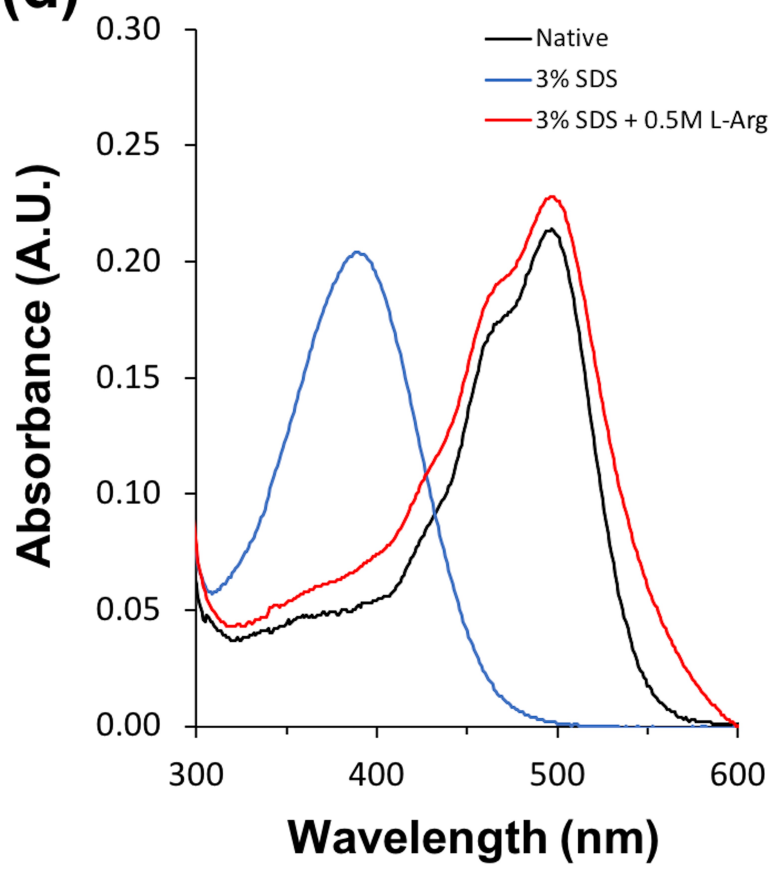




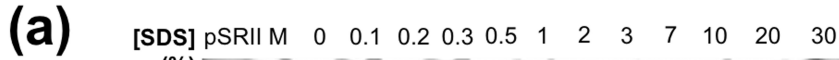

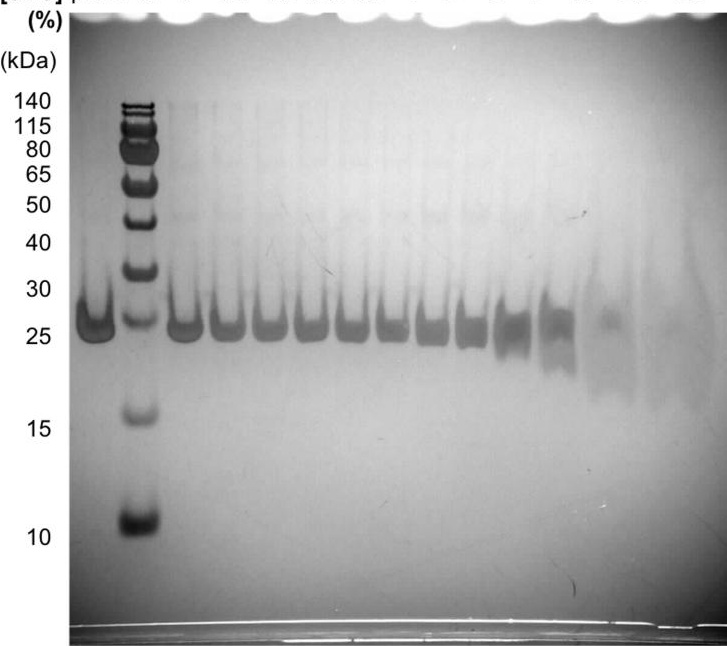

(b)
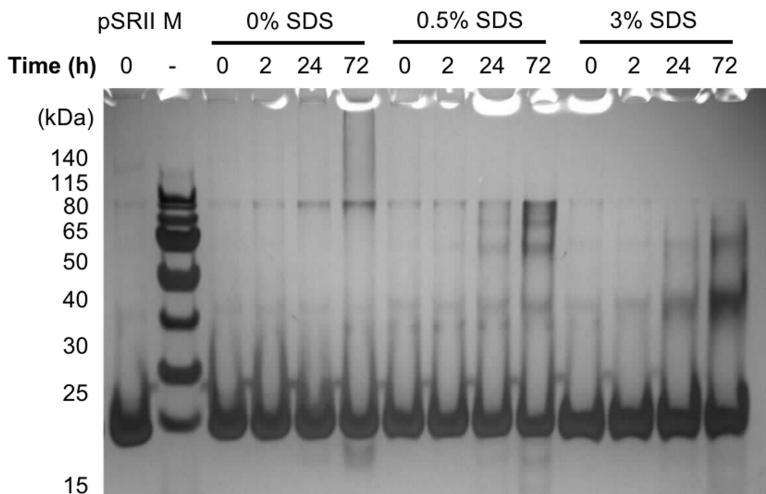

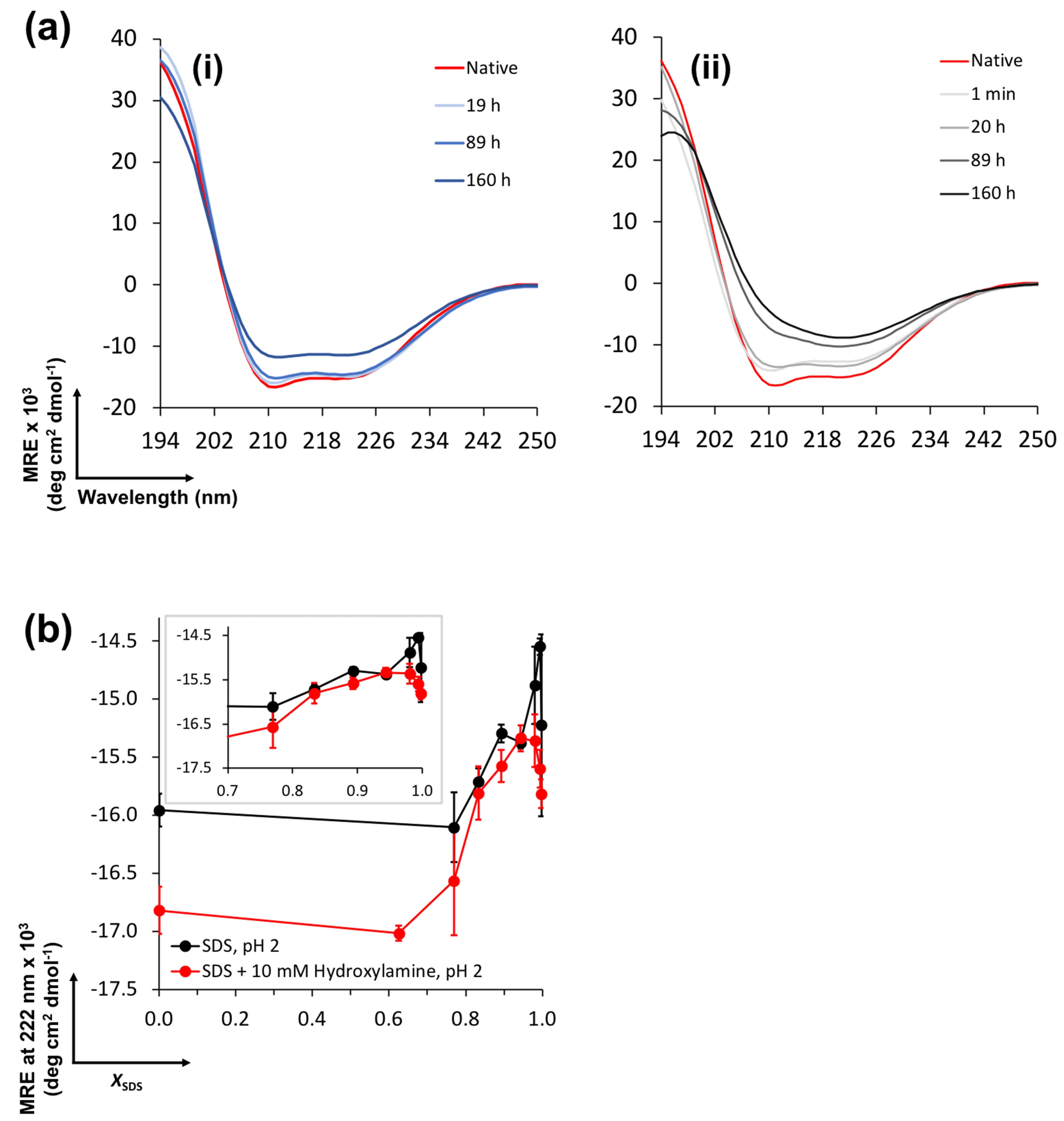
$H(r)$

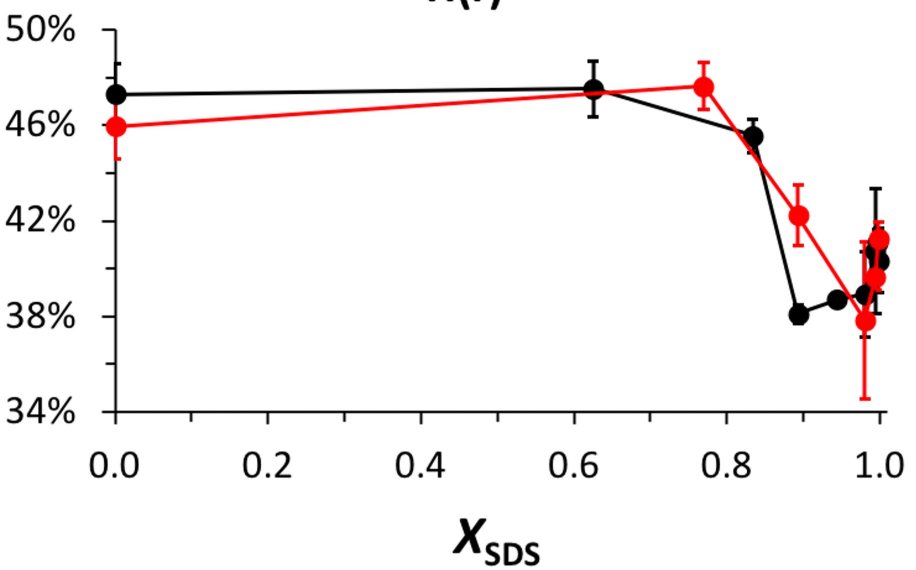

$S(r)$

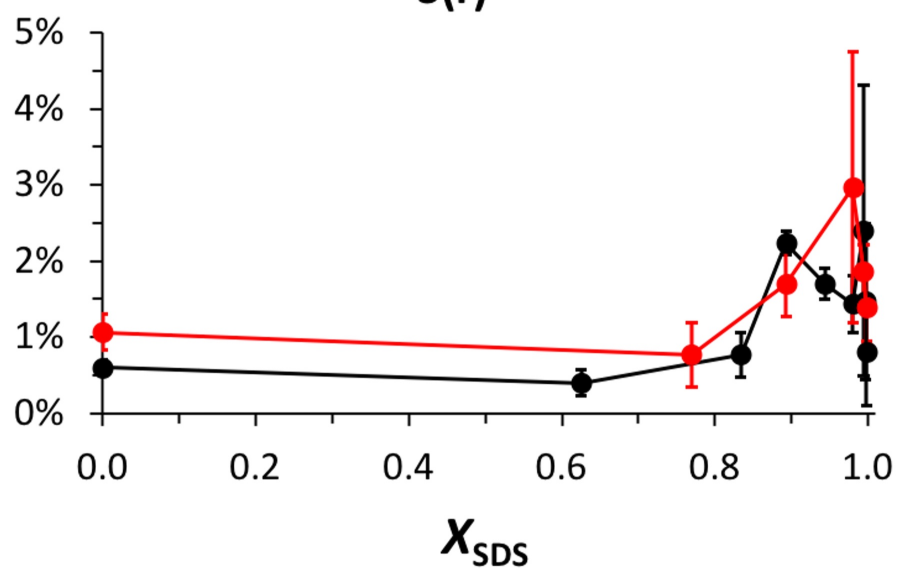

Turn

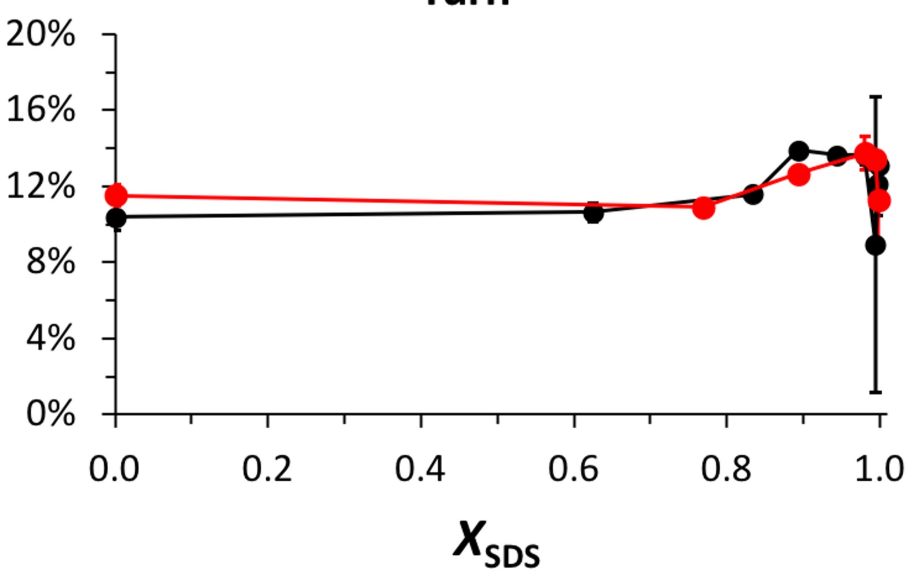

$H(d)$

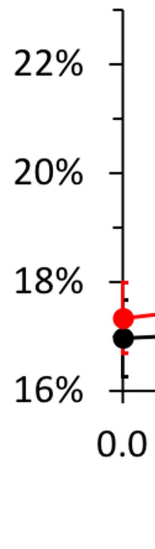

$-\mathrm{pH} 6.0$

-pH 2.0

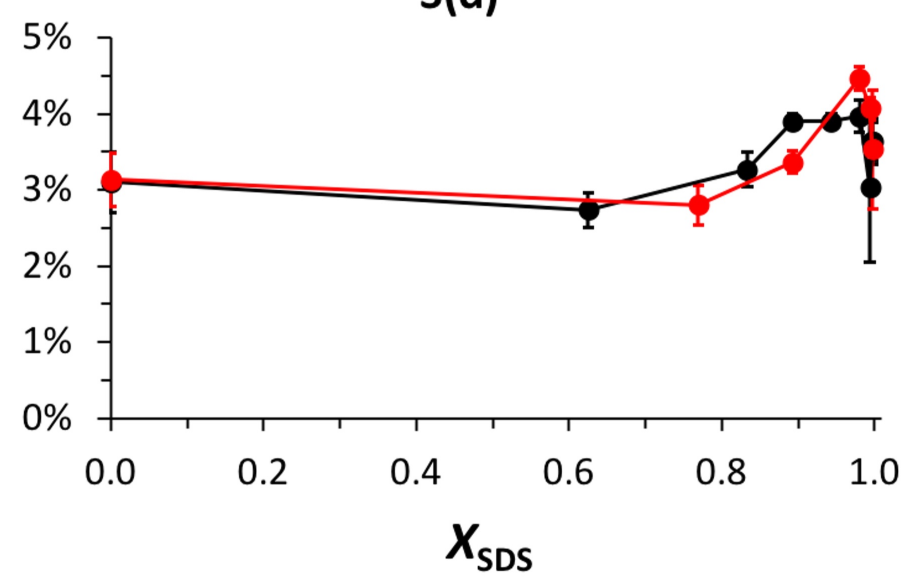

Unrd

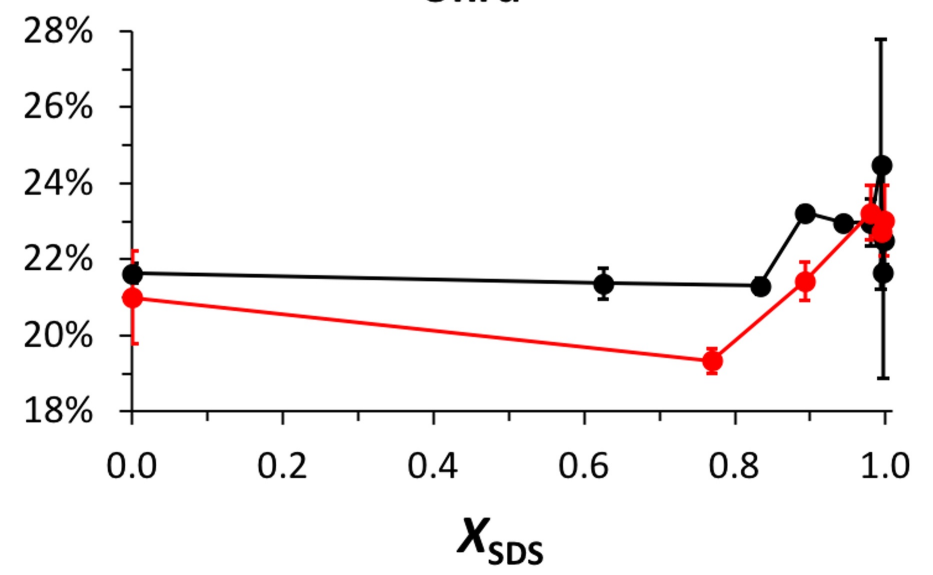


(a) $0.7 \quad 0 x_{\text {SDS }}$, pH 2.0

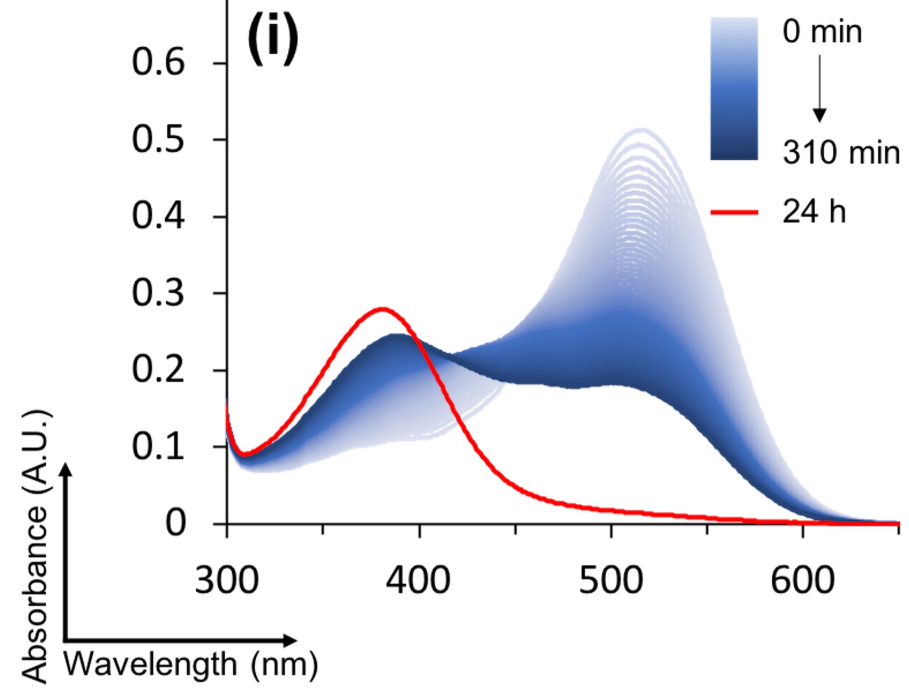

(b) $0 x_{\mathrm{SDS}}+10 \mathrm{mM} \mathrm{NH}_{2} \mathrm{OH}, \mathrm{pH} 2.0$

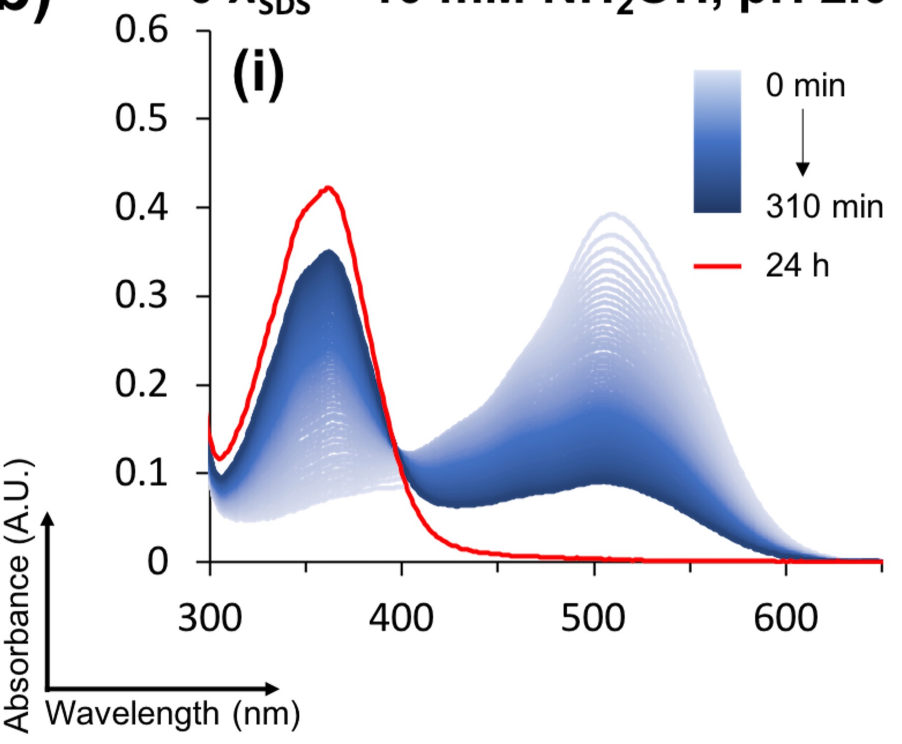

(c)

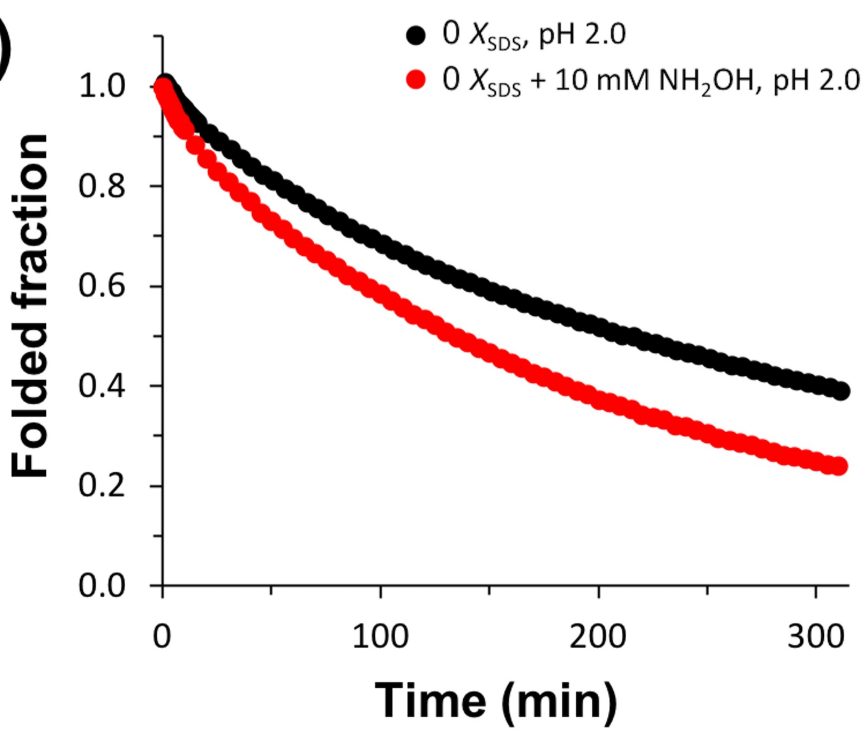

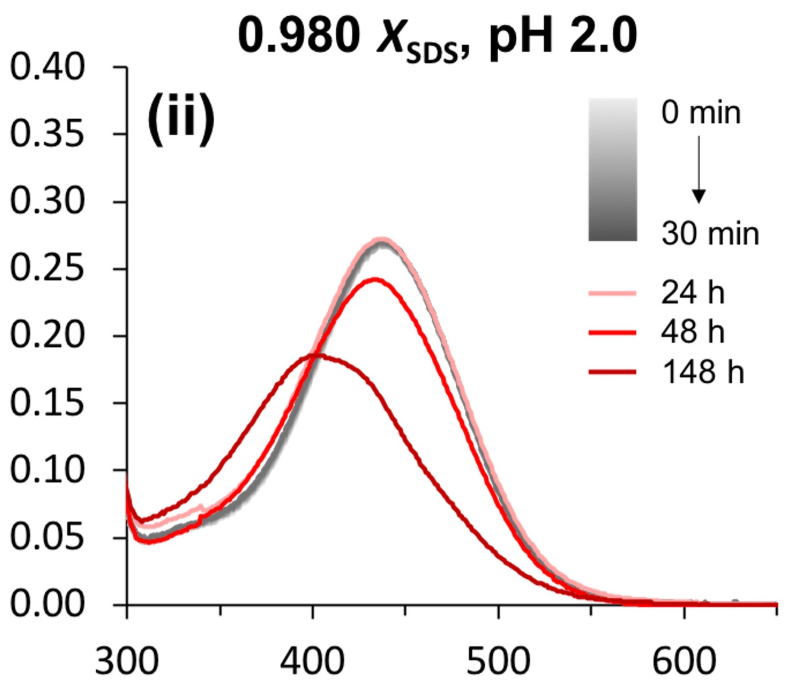

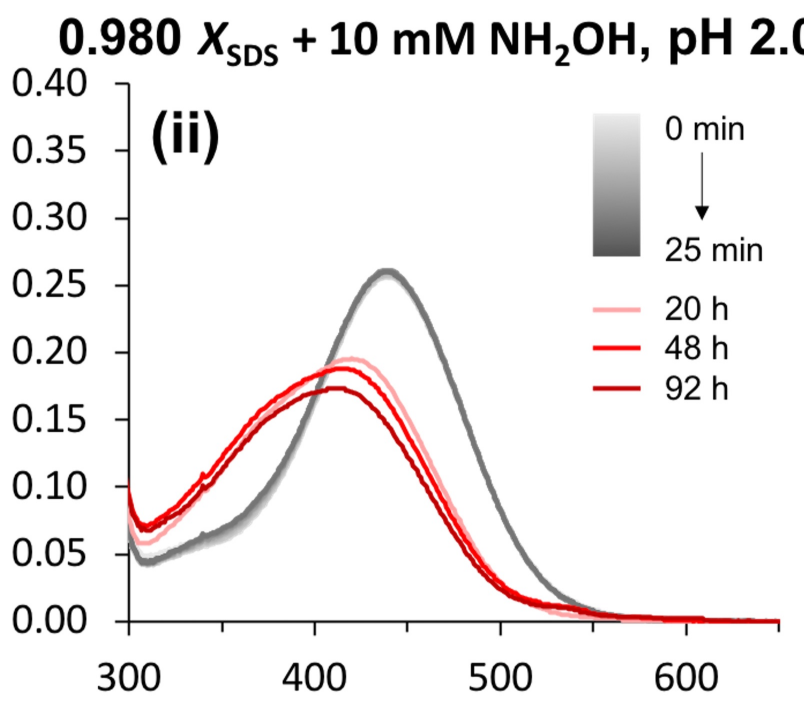




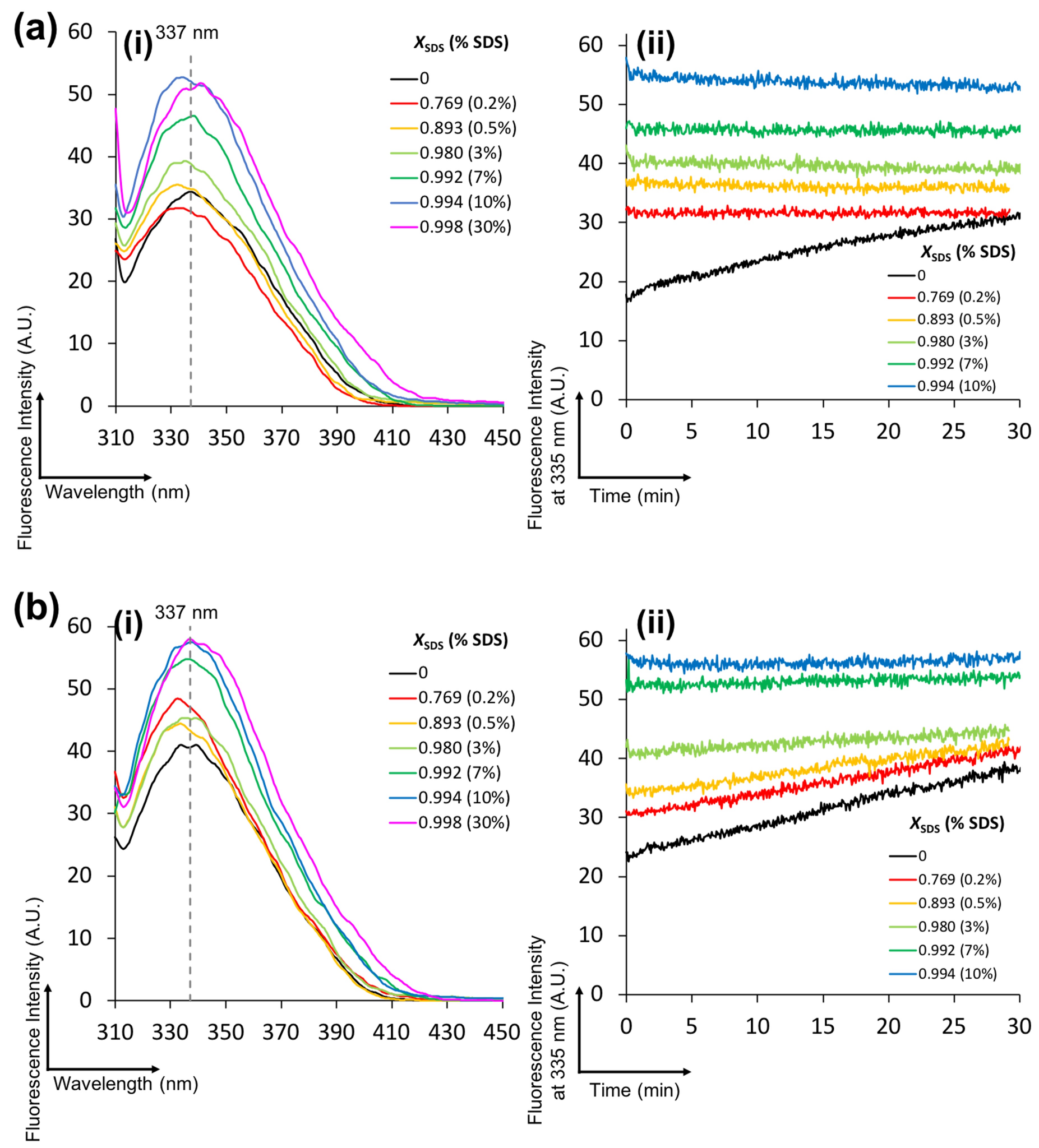




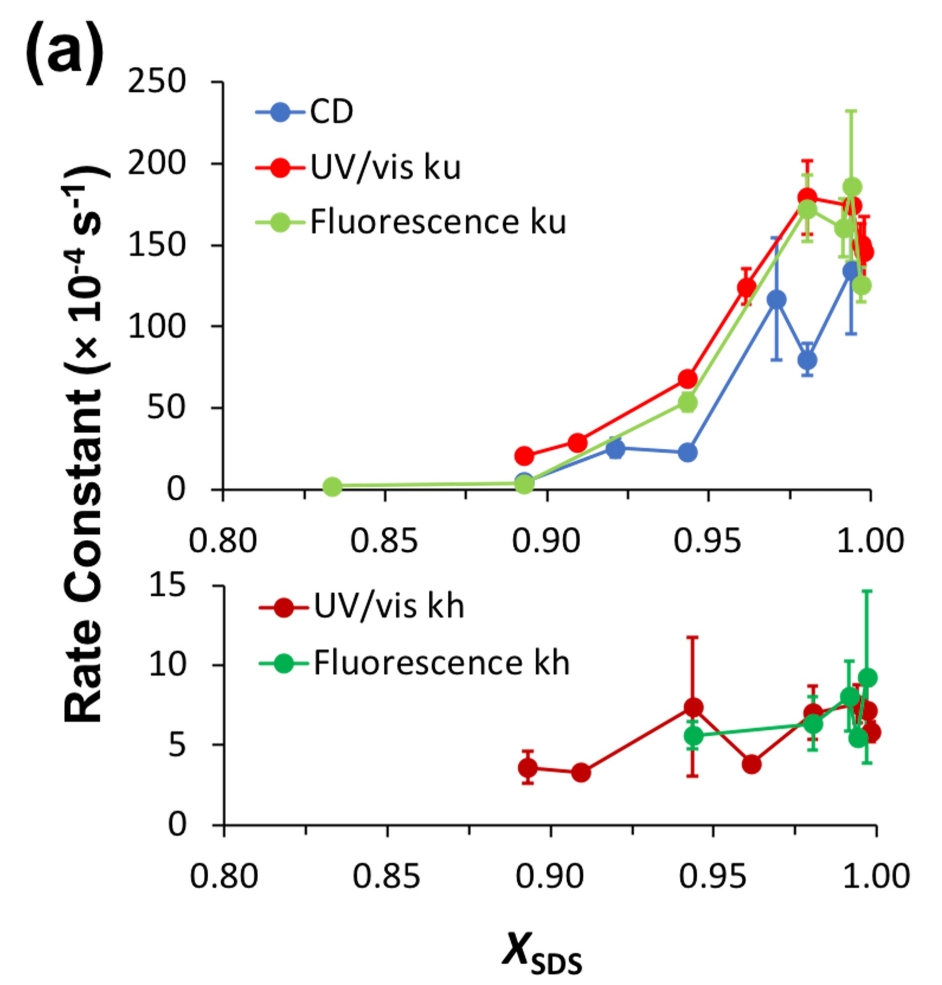

(b)

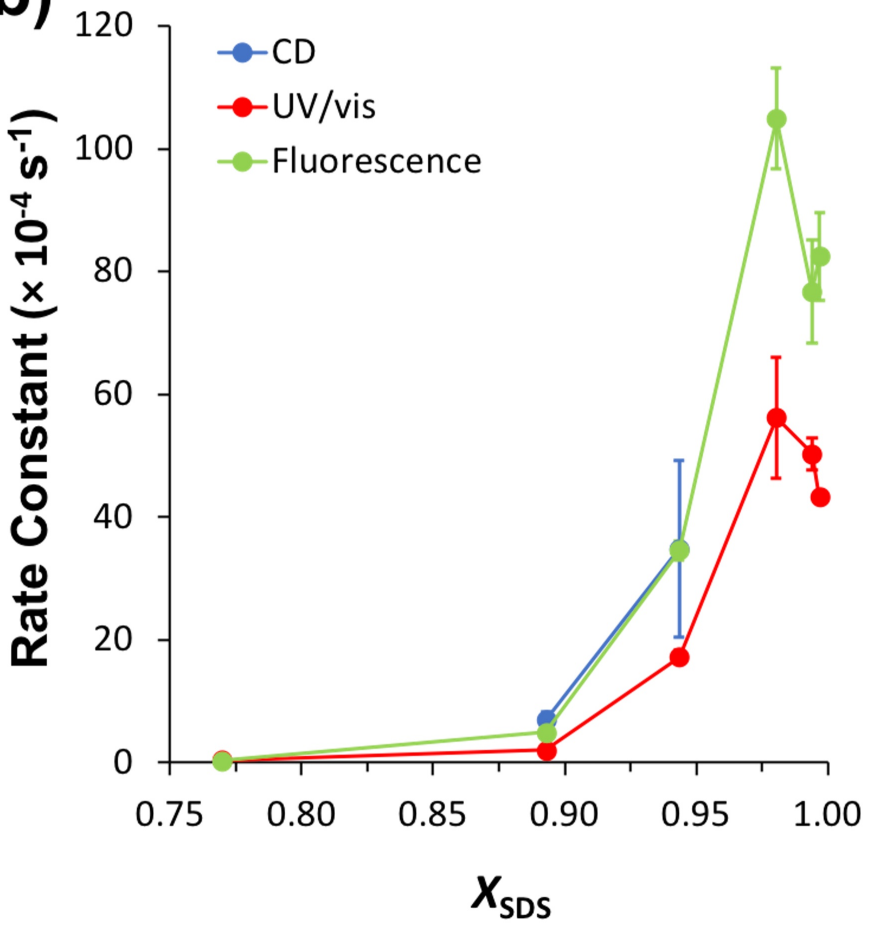


(a)

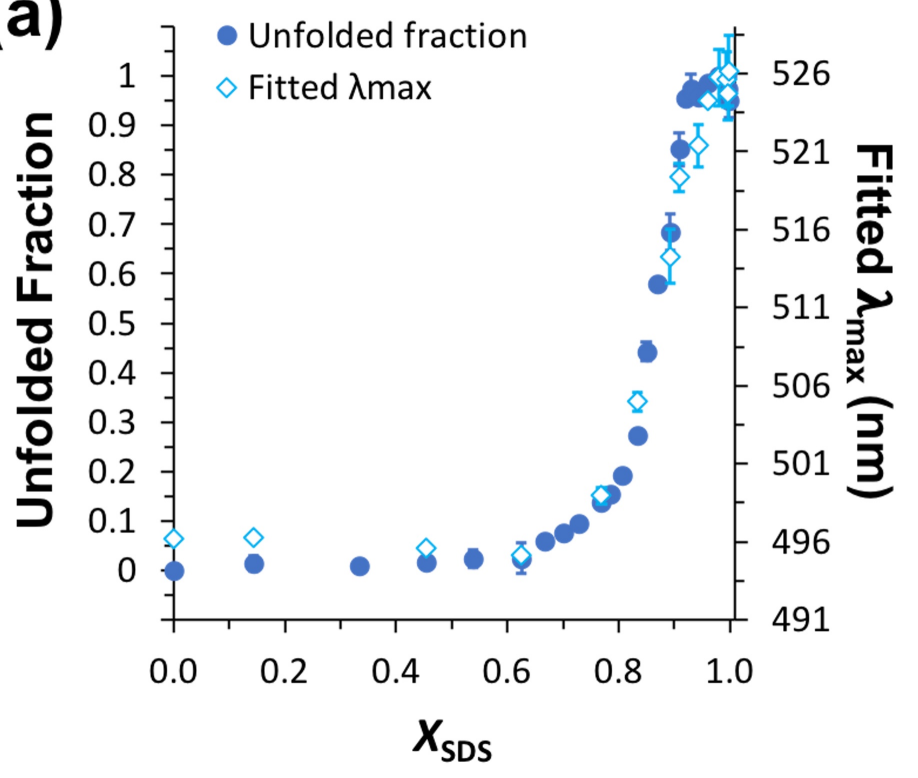

(b)

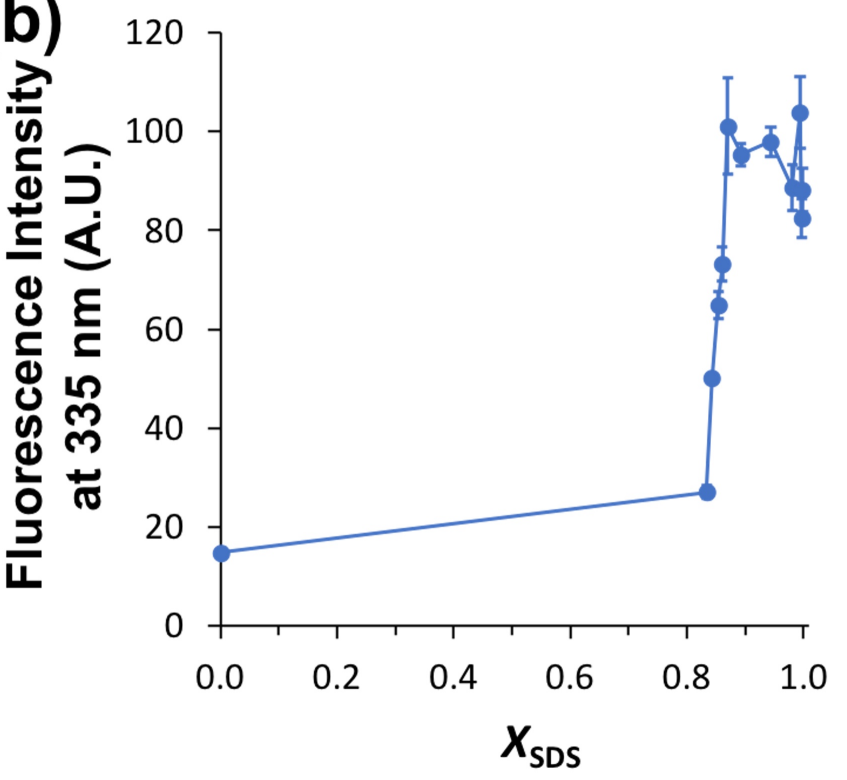



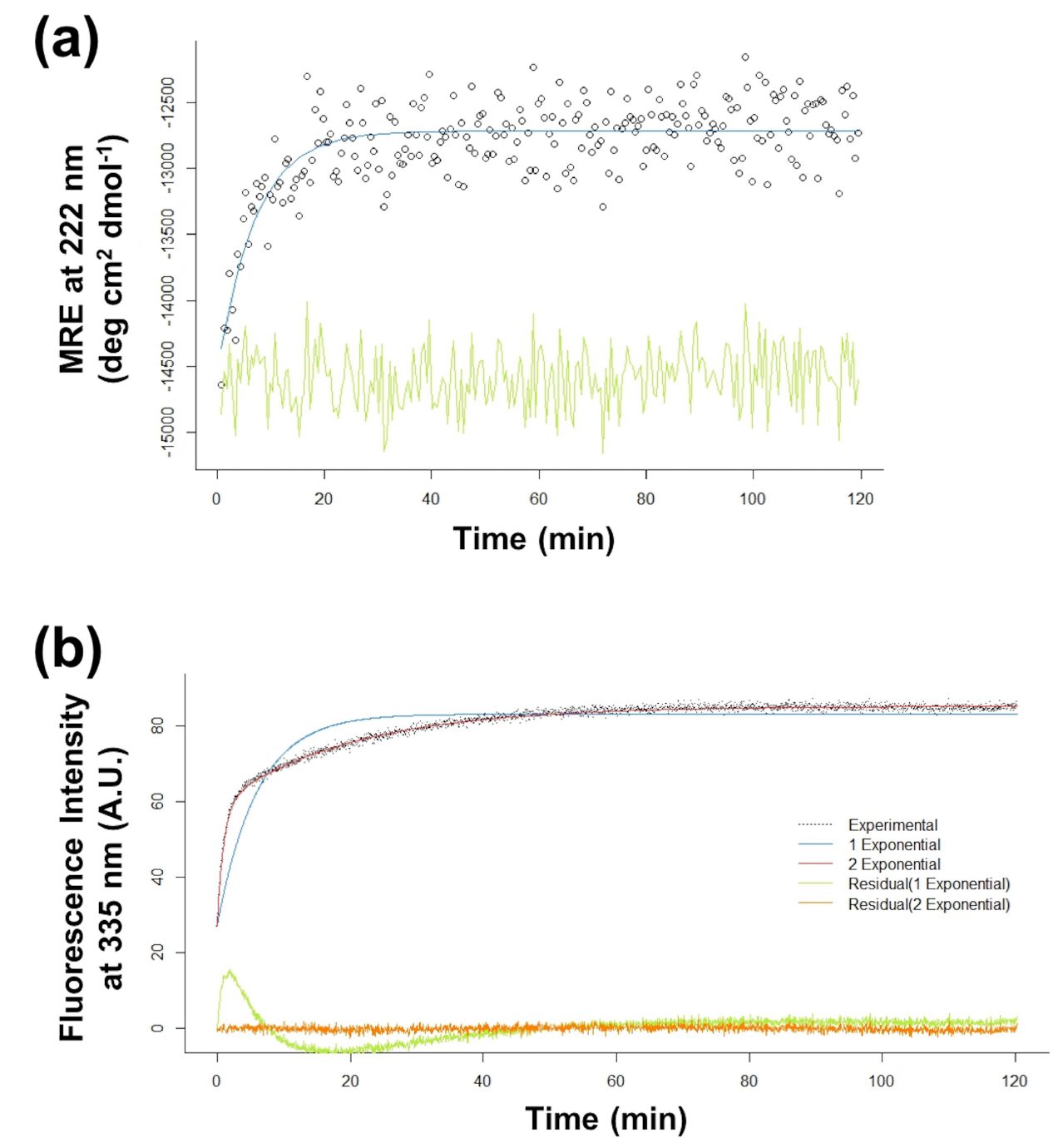

(c)

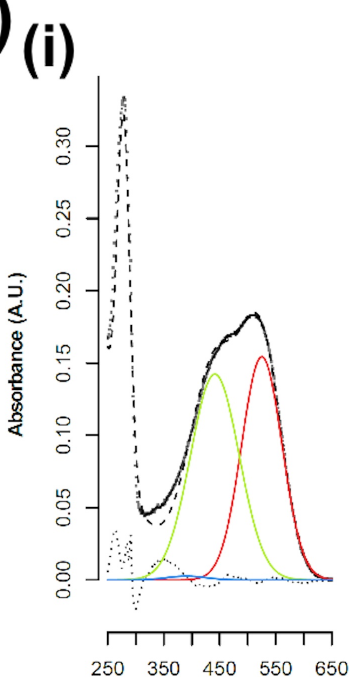

(ii)

(iii)

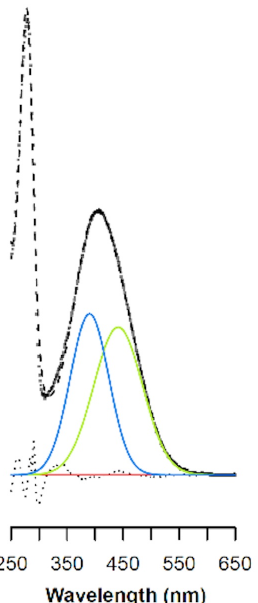

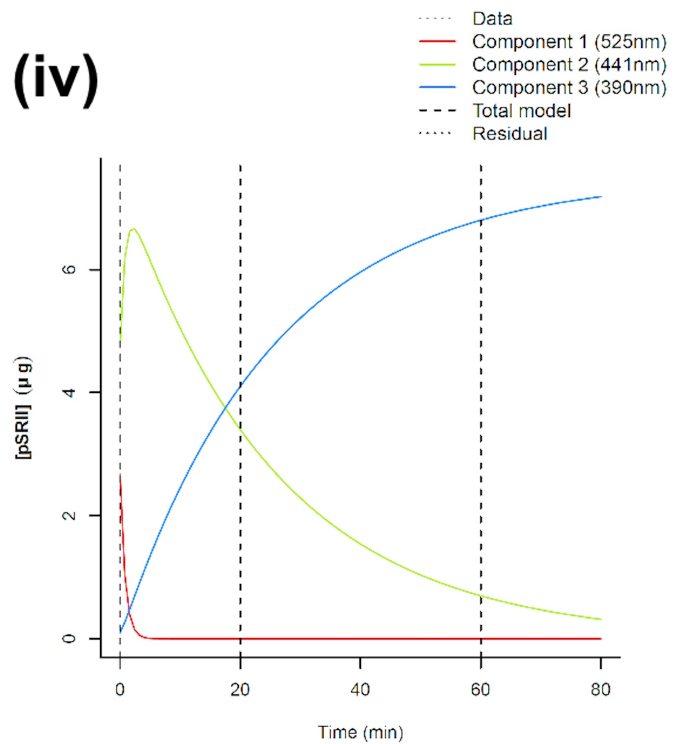




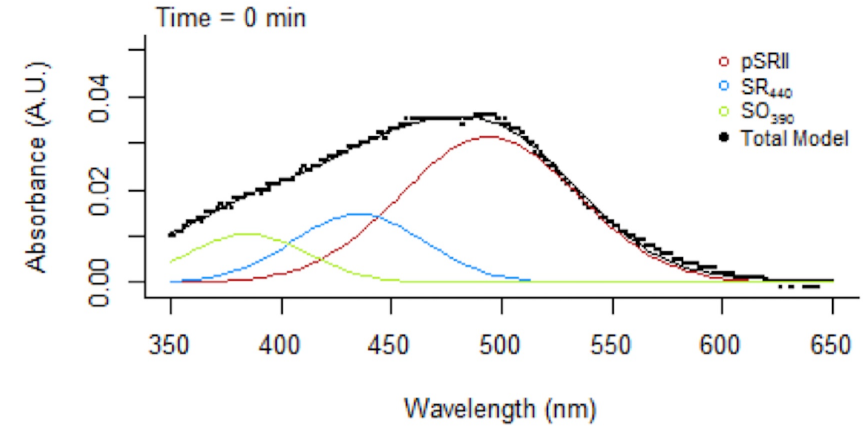

(b)

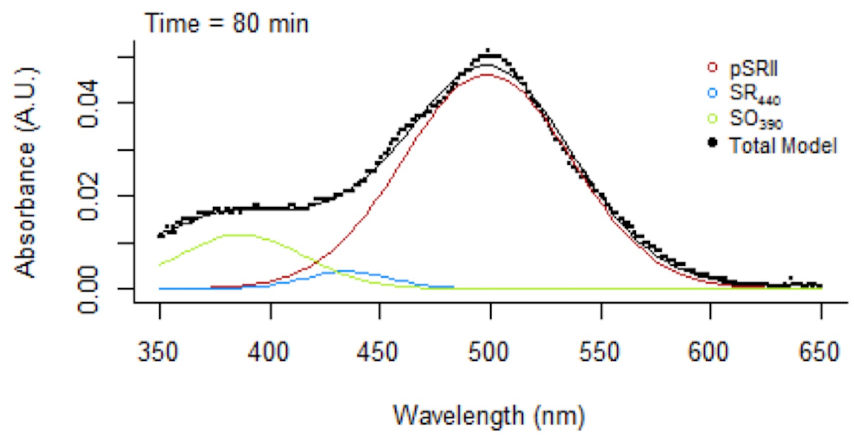

(d)

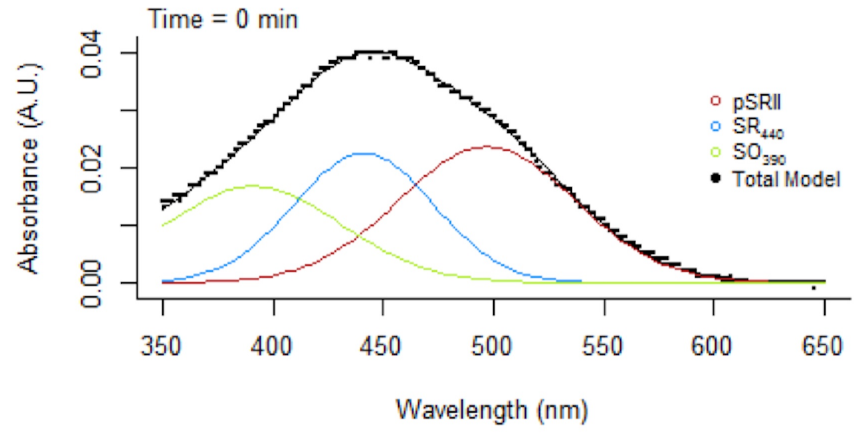

(e)

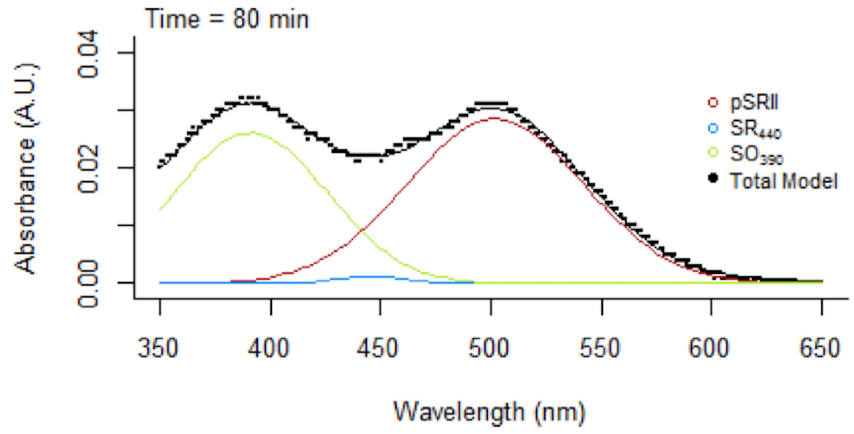

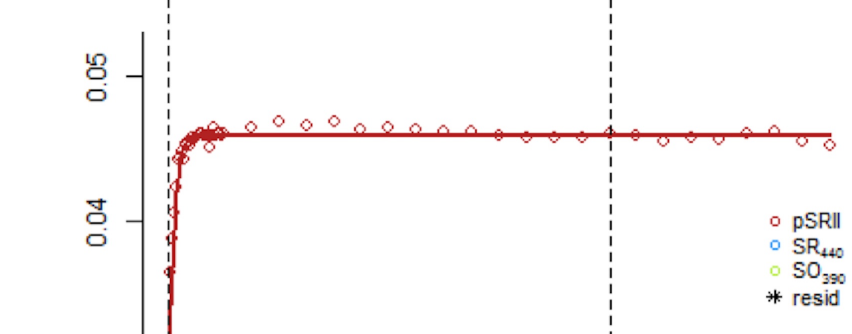

(f)

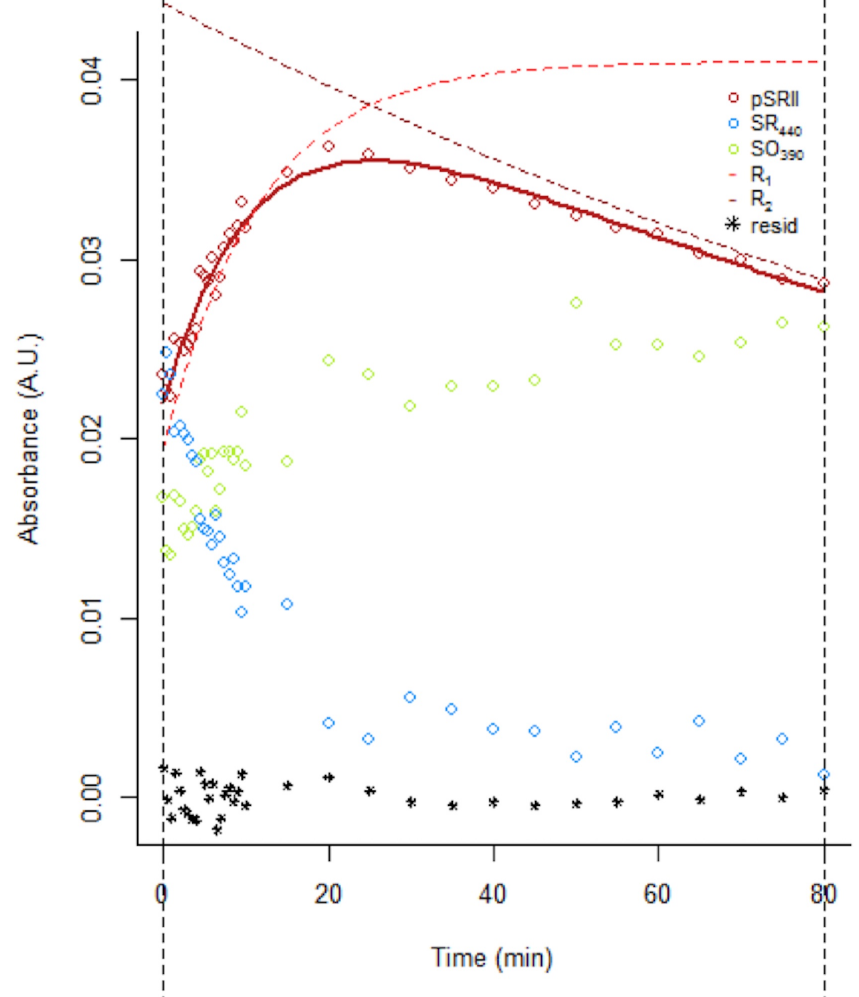


(a)

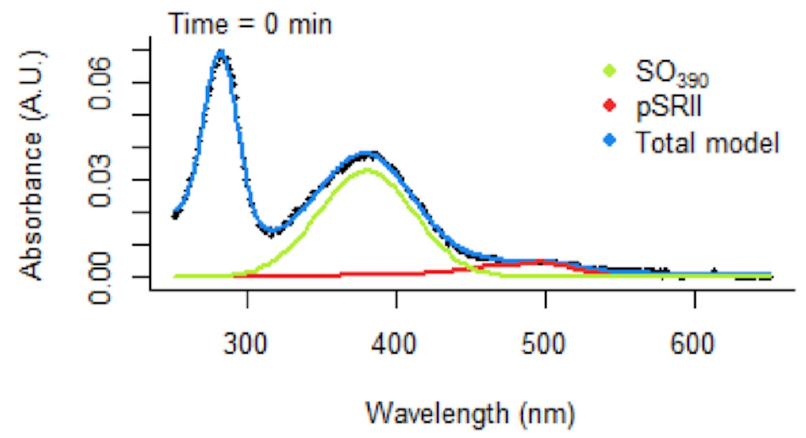

(b)

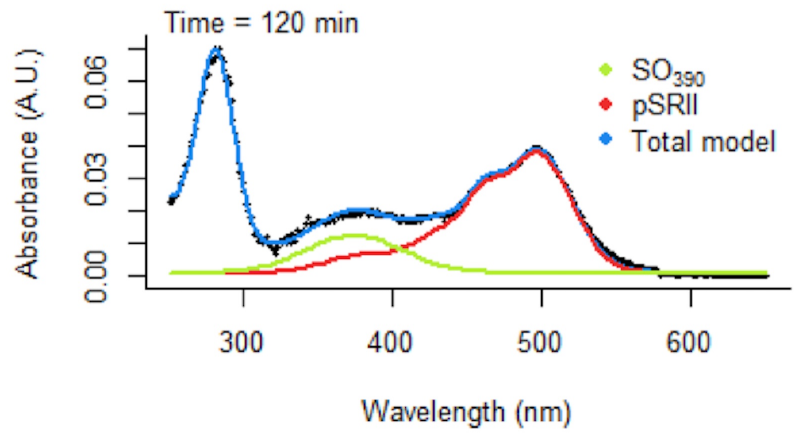

(c)

$\mathrm{SO}_{390}$

- pSRII

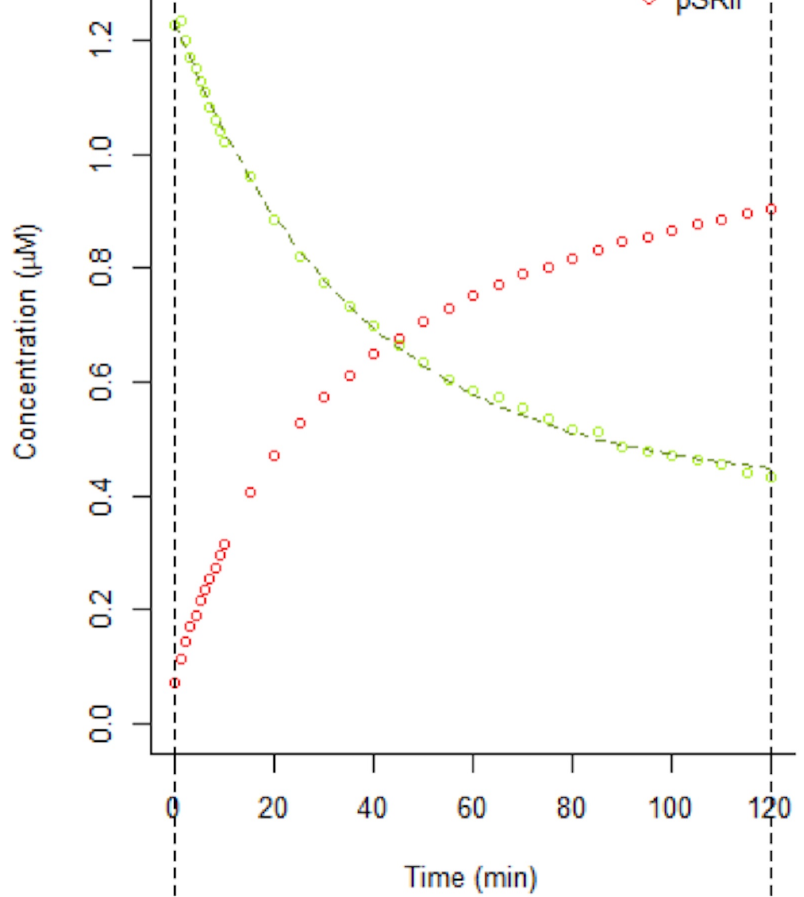


(a)

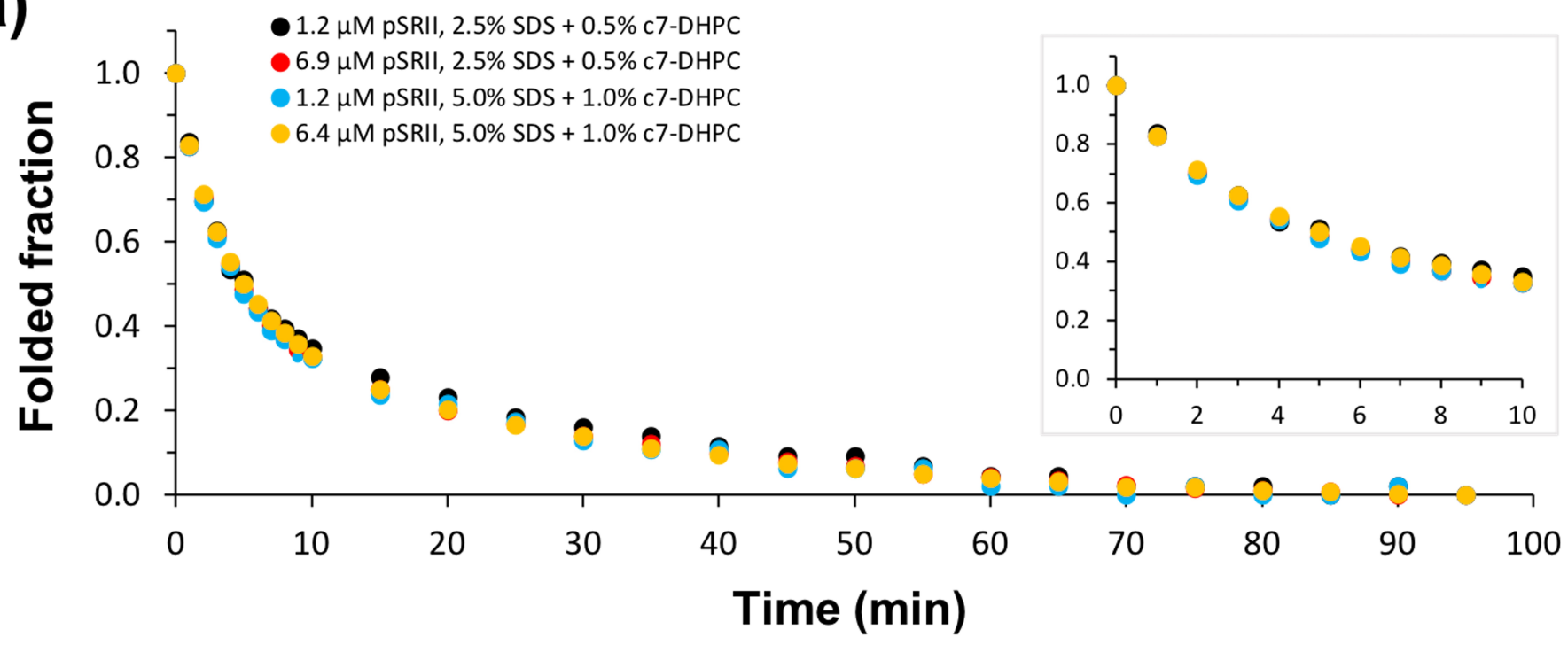

(b)

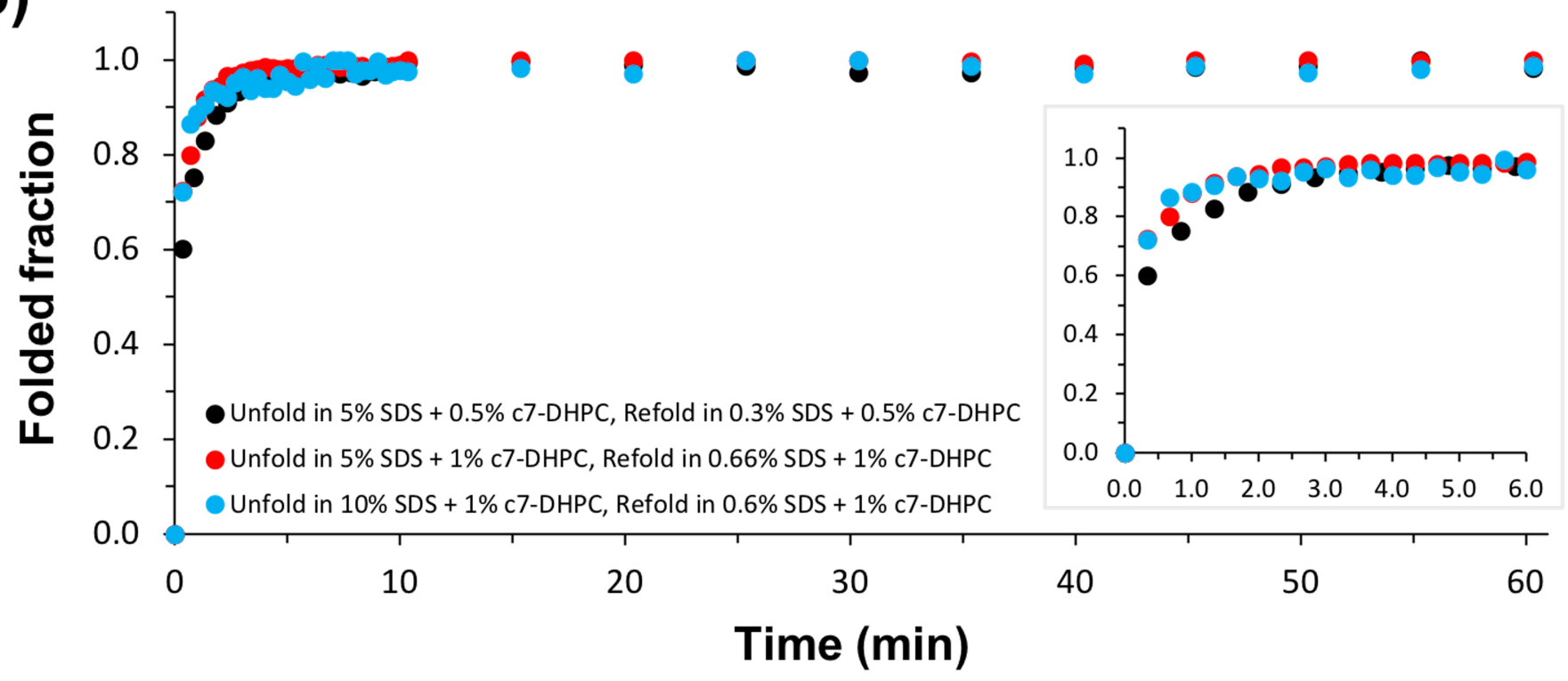

(c)

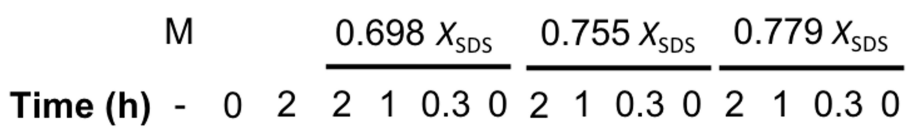

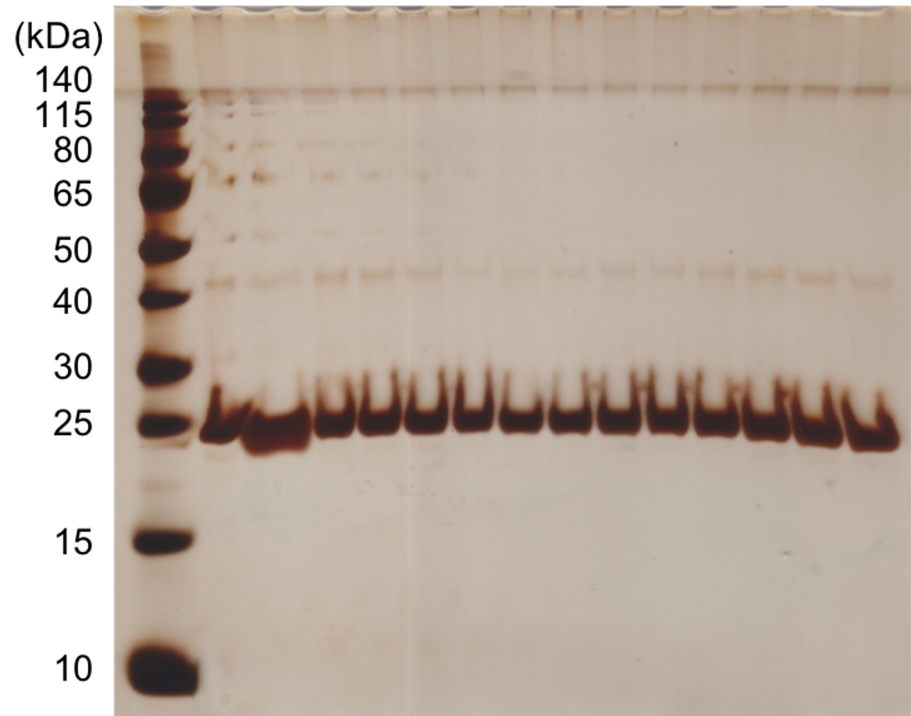




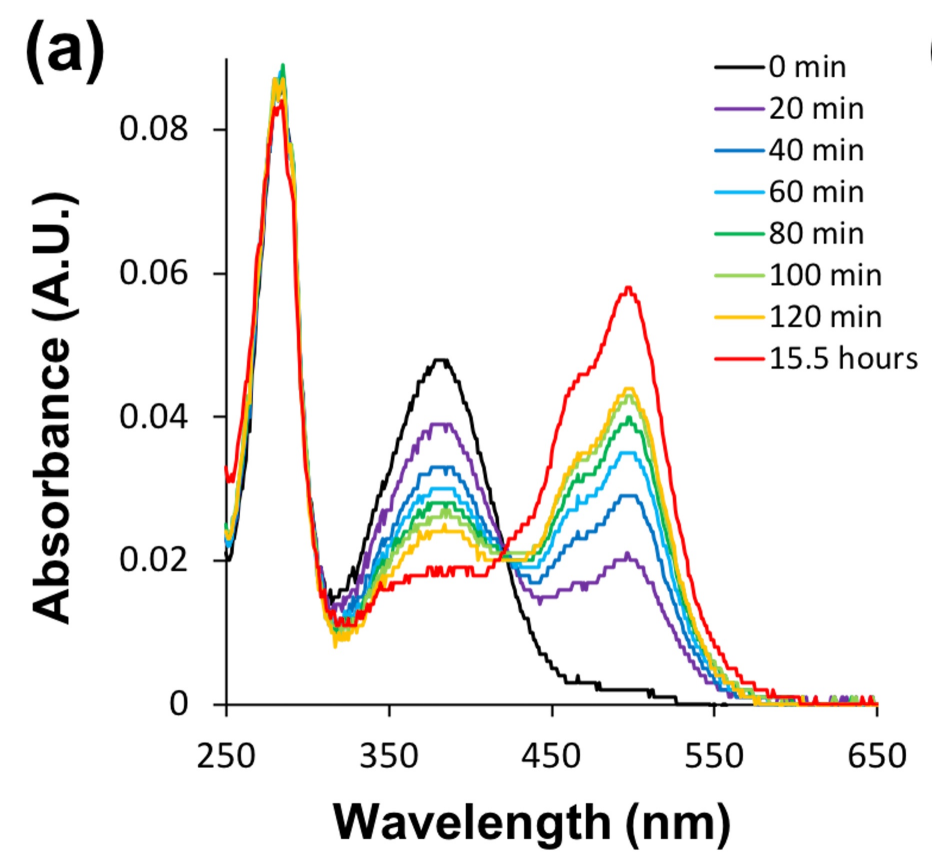

(b)

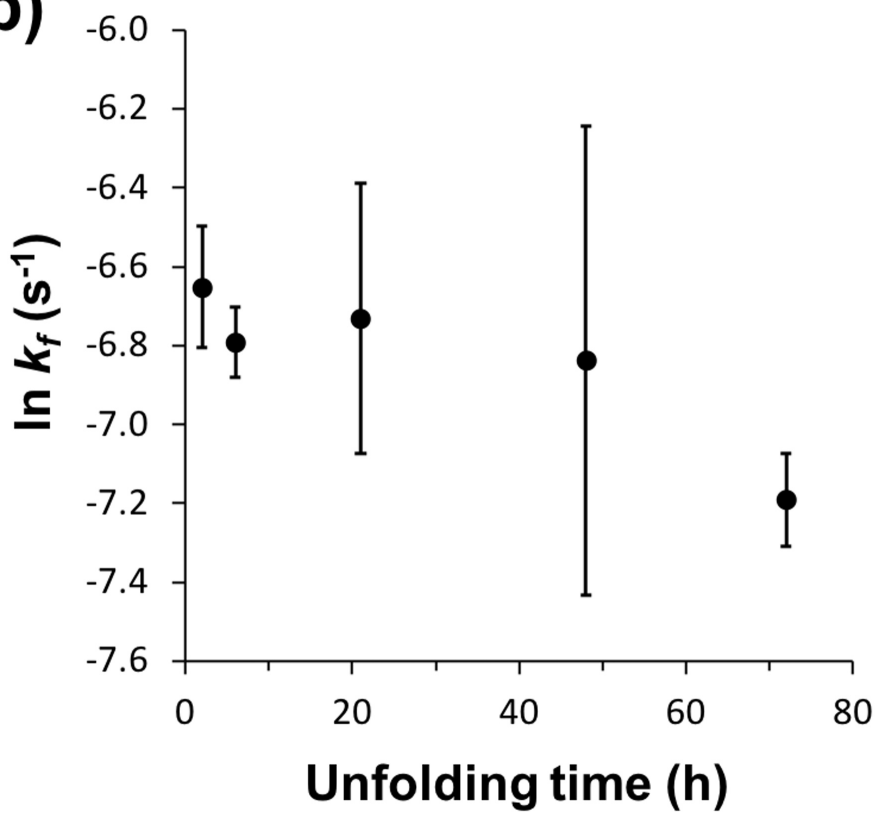




$$
\operatorname{lns}
$$

